\title{
P-C and C-C Bond Formation by Michael Addition in Platinum-Catalyzed Hydrophosphination and in the Stoichiometric Reactions of Platinum Phosphido Complexes with Activated Alkenes
}

\author{
Corina Scriban, David S. Glueck, * Lev N. Zakharov, W. Scott Kassel, Antonio G. DiPasquale, \\ James A. Golen, and Arnold L. Rheingold
}

\section{Supporting Information}

1. Additional experimental details

2. Details of the X-ray Structure Determinations

General Experimental. Experimental procedures and the preparation of starting materials are described in the manuscript text.

Pt((R,R)-Me-Duphos)(Me)(OMe). Under nitrogen, Na (115 mg, $5 \mathrm{mmol})$ was added to stirring $\mathrm{MeOH}$ (2.3 mL), which had been dried over molecular sieves. Gas evolved and the reaction was allowed to proceed until all the $\mathrm{Na}$ was consumed. $\mathrm{Pt}((\mathrm{R}, \mathrm{R})-\mathrm{Me}-\mathrm{Duphos})(\mathrm{Me})(\mathrm{Cl})(276 \mathrm{mg}$, $0.5 \mathrm{mmol})$ in toluene $(20 \mathrm{~mL})$ was added dropwise to the $\mathrm{NaOMe}$ stirring solution. The reaction mixture was heated to $\sim 60{ }^{\circ} \mathrm{C}$ for $\sim 48 \mathrm{~h}$. The solvent was removed under vacuum. The residue was extracted with toluene, then filtered through Celite. The filtrate was concentrated under vacuum, yielding a brown sticky residue. Petroleum ether was added to the residue giving a beige precipitate, which was washed with petroleum ether and dried under vacuum. Recrystallization from THF and petroleum ether yielded $184 \mathrm{mg}(67 \%)$ of beige powder. We could not isolate the methoxide complex in pure form, since it reacted with trace water to generate the hydroxide $\mathrm{Pt}((\mathrm{R}, \mathrm{R})-\mathrm{Me}-$ Duphos)(Me)(OH) (which was prepared independently, see below).

\footnotetext{
${ }^{31} \mathrm{P}\left\{{ }^{1} \mathrm{H}\right\} \operatorname{NMR}\left(\mathrm{C}_{6} \mathrm{D}_{6}\right): \delta 71.4\left(\mathrm{~d}, \mathrm{~J}=3, \mathrm{~J}_{\mathrm{Pt} \mathrm{P}}=1883\right), 52.8\left(\mathrm{~d}, \mathrm{~J}=3, \mathrm{~J}_{\mathrm{Pt} \mathrm{P}}=3226\right) .{ }^{1} \mathrm{H}$ NMR $\left(\mathrm{C}_{6} \mathrm{D}_{6}\right): \delta$ 7.18-7.10 (m, 2H, Ar), 7.01-6.98 (m, 2H, Ar), $4.77\left(\mathrm{~d}, \mathrm{~J}=6, \mathrm{~J}_{\mathrm{Pt}-\mathrm{H}}=47,3 \mathrm{H}, \mathrm{OCH}_{3}\right), 3.32-$
} 
$3.26(\mathrm{~m}, 1 \mathrm{H}, \mathrm{CH}), 2.79-2.75(\mathrm{~m}, 1 \mathrm{H}, \mathrm{CH}), 2.37-2.33(\mathrm{~m}, 1 \mathrm{H}, \mathrm{CH}), 2.27-2.22(\mathrm{~m}, 1 \mathrm{H}, \mathrm{CH}), 2.02-$ $1.66\left(\mathrm{~m}, 6 \mathrm{H}, \mathrm{CH}_{2}\right), 1.62(\mathrm{dd}, \mathrm{J}=19,7,3 \mathrm{H}, \mathrm{Me}), 1.28-1.18\left(\mathrm{~m}, 2 \mathrm{H}, \mathrm{CH}_{2}\right), 1.23(\mathrm{dd}, \mathrm{J}=18,7,3 \mathrm{H}$, Me), $1.14(\mathrm{dd}, \mathrm{J}=8,3,3 \mathrm{H}, \mathrm{Pt}-\mathrm{Me}), 0.68(\mathrm{dd}, \mathrm{J}=15,8,3 \mathrm{H}, \mathrm{Me}), 0.63(\mathrm{dd}, \mathrm{J}=15,7,3 \mathrm{H}, \mathrm{Me})$.

$\operatorname{Pt}((\mathbf{R}, \mathbf{R})-$ Me-Duphos$)(\mathbf{M e})(\mathbf{O H}) . \mathrm{NaOH}(82 \mathrm{mg}, 2.05 \mathrm{mmol})$ was added to a stirring slurry of $\mathrm{Pt}((\mathrm{R}, \mathrm{R})-\mathrm{Me}-\mathrm{Duphos})(\mathrm{Me})(\mathrm{Cl})(227 \mathrm{mg}, 0.41 \mathrm{mmol})$ in THF $(20 \mathrm{~mL})$, with stirring. Distilled $\mathrm{H}_{2} \mathrm{O}(2 \mathrm{~mL})$ was added to help dissolve the $\mathrm{NaOH}$. After $48 \mathrm{~h}$, the solvent was removed under vacuum and toluene $(20 \mathrm{~mL})$ was added to the residue. The yellow slurry was filtered through Celite, and the filtrate was concentrated under vacuum to give a yellow solid. ${ }^{31} \mathrm{P}\left\{{ }^{1} \mathrm{H}\right\} \mathrm{NMR}$ $\left(\mathrm{C}_{6} \mathrm{D}_{6}\right): \delta 70.3\left(\mathrm{~d}, \mathrm{~J}=3, \mathrm{~J}_{\mathrm{Pt}-\mathrm{P}}=1871\right), 52.9\left(\mathrm{~d}, \mathrm{~J}=3, \mathrm{~J}_{\mathrm{Pt}-\mathrm{P}}=3229\right)$.

Alternative Synthesis of $\mathbf{P t}((\mathbf{R}, \mathbf{R})-\mathrm{Me}-\mathrm{Duphos})(\mathbf{M e})\left(\mathbf{P P h}_{2}\right)$ (11). $\mathrm{PHPh}_{2}(50 \mathrm{mg}, 0.27$ mmol) was added with a microsyringe to a stirring slurry of $\operatorname{Pt}((\mathrm{R}, \mathrm{R})-\mathrm{Me}-\mathrm{Duphos})(\mathrm{Me})(\mathrm{OMe})$ (145 mg, $0.27 \mathrm{mmol})$ in toluene $(20 \mathrm{~mL})$. As soon as $\mathrm{Pt}((\mathrm{R}, \mathrm{R})-\mathrm{Me}-\mathrm{Duphos})(\mathrm{Me})(\mathrm{OMe})$ reacted, the mixture turned yellow immediately. After stirring for $12 \mathrm{~h}$, the slurry was filtered through Celite, and the yellow filtrate was concentrated under vacuum. Petroleum ether was added to the yellow residue, yielding a yellow precipitate, which was washed with petroleum ether. Drying the precipitate yielded $158 \mathrm{mg}(87 \%)$ of yellow powder. Recrystallization from THF and petroleum ether gave orange-yellow crystals, suitable for X-ray crystallography. 


\section{Reaction of the Complexes $\operatorname{Pt}(\operatorname{diphos})\left(R^{\prime}\right)\left(P R_{2}\right)$ with Acrylonitrile and $t$-Butyl}

Acrylate (Scheme 6). Table S1 lists the observed products. Experimental details for a few of the entries in Table S1 were reported in the Experimental section of the manuscript; the rest are included below.

Scheme 6 Reactions of $\mathrm{Pt}($ diphos $)\left(\mathrm{R}^{\prime}\right)\left(\mathrm{PR}_{2}\right)$ Complexes with $t$-Butyl Acrylate and Acrylonitrile $\left([\mathrm{Pt}]=\mathrm{Pt}((\mathrm{R}, \mathrm{R})-\mathrm{Me}-\mathrm{Duphos}), \mathrm{R}^{\prime}=\mathrm{Me}, \mathrm{PR}_{2}=\mathrm{PPh}_{2}(\mathbf{1 1}), \mathrm{PPh}\left(i\right.\right.$-Bu) (12); $\mathrm{R}^{\prime}=\mathrm{Ph}, \mathrm{PR}_{2}=\mathrm{PMeIs}$ (13); $[\mathrm{Pt}]=\mathrm{Pt}(\mathrm{dppe}), \mathrm{R}^{\prime}=\mathrm{Me}, \mathrm{PR}_{2}=\mathrm{PPh}_{2}(\mathbf{1 4}), \mathrm{PPh}(i-\mathrm{Bu})(\mathbf{1 5}), \mathrm{X}=\mathrm{CN}$ or $\mathrm{CO}_{2} t-\mathrm{Bu}$. Complex C could not be identified; a tentative formulation is shown (see the text for discussion)). For $\mathbf{1 7}$, $[\mathrm{Pt}]=\mathrm{Pt}(\mathrm{dppe}), \mathrm{R}=\mathrm{Ph}(\mathbf{1 7 a})$ or $i-\mathrm{Bu}(\mathbf{1 7 b}) ;[\mathrm{Pt}]=\mathrm{Pt}(\mathrm{R}, \mathrm{R})-\mathrm{Me}-\mathrm{Duphos}), \mathrm{R}=i$-Bu. 
Table S1. Products Observed in the Reactions of $\mathrm{Pt}($ diphos $)\left(\mathrm{R}^{\prime}\right)\left(\mathrm{PR}_{2}\right)$ Complexes with $t$-Butyl Acrylate and Acrylonitrile (Scheme 6) ${ }^{\mathrm{a}}$

\begin{tabular}{|c|c|c|c|c|c|c|c|c|c|c|c|c|c|}
\hline Entry & Temp $^{b}$ & Diphos & $\mathbf{X}$ & $\mathbf{R}^{\prime}$ & $\mathbf{P R}_{2}$ & $\mathbf{A}$ & $\overline{\mathbf{B}}$ & $\mathbf{C}$ & 3 & $77_{1}^{c}$ & $77^{c}$ & 16 & 17 \\
\hline 1 & low & dppe & $\mathrm{CO}_{2} t-\mathrm{Bu}$ & $\mathrm{Me}$ & $\mathrm{PPh}_{2}$ & $\bullet^{\mathrm{d}}$ & $\bullet$ & $\bullet^{e}$ & $\bullet$ & $\bullet$ & $e^{e}$ & $e^{e}$ & \\
\hline $2^{1}$ & $\mathrm{RT}$ & dppe & $\mathrm{CO}_{2} t-\mathrm{Bu}$ & Me & $\mathrm{PPh}_{2}$ & & $\bullet$ & $\bullet$ & $\bullet$ & $\bullet$ & $\bullet^{\mathrm{g}}$ & $\bullet^{\mathrm{g}}$ & \\
\hline $3^{\mathrm{h}, 1}$ & $\overline{\mathrm{RT}}$ & dppe & $\mathrm{CO}_{2} t-\mathrm{Bu}$ & $\mathrm{Me}$ & $\mathrm{PPh}_{2}$ & & $\bullet$ & $\bullet$ & $\bullet$ & $\bullet$ & & & \\
\hline $4^{\mathrm{j}, 1}$ & $\overline{\mathrm{RT}}$ & dppe & $\mathrm{CO}_{2} t-\mathrm{Bu}$ & Me & $\mathrm{PPh}_{2}$ & & & & $\bullet$ & $\bullet$ & $\bullet$ & & $\bullet$ \\
\hline $5^{1}$ & $\overline{\mathrm{RT}}$ & dppe & $\overline{\mathrm{CN}}$ & $\mathrm{Me}$ & $\mathrm{PPh}_{2}$ & $\bullet$ & $\bullet$ & & $\bullet$ & $\bullet^{\mathrm{K}}$ & & & $\bullet^{\mathrm{K}}$ \\
\hline $6^{1}$ & $\overline{\mathrm{RT}}$ & dppe & $\overline{\mathrm{CN}}$ & $\overline{\mathrm{Me}}$ & $\mathrm{PPh}_{2}$ & $\bullet$ & $\bullet$ & $\bullet$ & $\bullet$ & $\bullet$ & & & $\bullet$ \\
\hline $7^{m}$ & $\overline{\mathrm{RT}}$ & dppe & $\mathrm{CN}$ & $\overline{\mathrm{Me}}$ & $\mathrm{PPh}_{2}$ & $\bullet$ & $\bullet$ & & $\bullet$ & $\bullet$ & & & \\
\hline $8^{n, 1}$ & $\mathrm{RT}$ & dppe & $\mathrm{CN}$ & $\mathrm{Me}$ & $\mathrm{PPh}_{2}$ & $\bullet$ & $\bullet$ & & $\bullet$ & $\bullet$ & $\bullet^{\mathrm{g}}$ & & $\bullet^{\mathrm{g}}$ \\
\hline $9^{0,1}$ & $\overline{\mathrm{RT}}$ & dppe & $\mathrm{CO}_{2} t-\mathrm{Bu}$ & $\mathrm{Me}$ & $\operatorname{PPh}(i-\mathrm{Bu})$ & & $\bullet$ & $\bullet$ & $\bullet$ & $\bullet$ & $\bullet^{\mathrm{g}}$ & $\bullet^{\mathrm{g}}$ & $\bullet^{\mathrm{g}}$ \\
\hline $10^{\mathrm{p}, 1}$ & $\mathrm{RT}$ & dppe & $\mathrm{CO}_{2} t-\mathrm{Bu}$ & $\mathrm{Me}$ & $\operatorname{PPh}(i-\mathrm{Bu})$ & & $\bullet$ & & $\bullet$ & $\bullet$ & & $\bullet$ & $\bullet$ \\
\hline $11^{\mathrm{j}}$ & RT & dppe & $\mathrm{CO}_{2} t-\mathrm{Bu}$ & $\mathrm{Me}$ & $\operatorname{PPh}(i-\mathrm{Bu})$ & & & & & $\bullet$ & $\bullet$ & & $\bullet$ \\
\hline 12 & low & $(\mathrm{R}, \mathrm{R})-\mathrm{Me}-\mathrm{Duphos}$ & $\mathrm{CO}_{2} t-\mathrm{Bu}$ & $\mathrm{Me}$ & $\mathrm{PPh}_{2}$ & $\bullet^{9}$ & $\bullet$ & & $\bullet$ & $\bullet$ & & & \\
\hline 13 & $\overline{\mathrm{RT}}$ & (R,R)-Me-Duphos & $\mathrm{CO}_{2} t-\mathrm{Bu}$ & $\mathrm{Me}$ & $\mathrm{PPh}_{2}$ & & $\bullet$ & & $\bullet$ & $\bullet$ & & $\bullet^{\mathrm{g}}$ & \\
\hline $14^{\mathrm{r}}$ & $\overline{\mathrm{RT}}$ & (R,R)-Me-Duphos & $\mathrm{CO}_{2} t-\mathrm{Bu}$ & $\mathrm{Me}$ & $\mathrm{PPh}_{2}$ & & $\bullet$ & $\circ 9$ & $\bullet$ & $\bullet$ & & & \\
\hline 15 & low & (R,R)-Me-Duphos & $\mathrm{CO}_{2} t-\mathrm{Bu}$ & $\mathrm{Me}$ & $\mathrm{PPh}(i-\mathrm{Bu})$ & $\bullet^{d}$ & $\bullet$ & $\bullet$ & $\bullet$ & $\bullet$ & & & \\
\hline 16 & RT & (R,R)-Me-Duphos & $\mathrm{CO}_{2} t-\mathrm{Bu}$ & $\mathrm{Me}$ & $\operatorname{PPh}(i-\mathrm{Bu})$ & & $\bullet$ & & $\bullet$ & $\bullet$ & & $\bullet$ & \\
\hline $17^{\mathrm{s}}$ & $\mathrm{RT}$ & (R,R)-Me-Duphos & $\mathrm{CO}_{2} t-\mathrm{Bu}$ & $\mathrm{Me}$ & $\operatorname{PPh}(i-\mathrm{Bu})$ & & $\bullet$ & $\bullet$ & & $\bullet$ & & & $\bullet$ \\
\hline $18^{t}$ & RT & (R,R)-Me-Duphos & $\mathrm{CO}_{2} t-\mathrm{Bu}$ & $\mathrm{Me}$ & $\operatorname{PPh}(i-\mathrm{Bu})$ & & & $\bullet$ & $\bullet$ & $\bullet$ & & & \\
\hline $19^{\mathrm{u}}$ & low & (R,R)-Me-Duphos & $\mathrm{CO}_{2} t-\mathrm{Bu}$ & $\mathrm{Ph}$ & PMeIs & $\bullet$ & & $\bullet^{e}$ & $\bullet$ & $\bullet$ & & & \\
\hline
\end{tabular}

${ }^{a}$ See the experimental section and below for procedures and details. The Pt:alkene ratio was 1:1, unless indicated. ${ }^{b}$ The reagents were combined either at room temperature (RT) or at low temperature and the reactions were monitored by ${ }^{31} \mathrm{P}$ and ${ }^{1} \mathrm{H}$ NMR spectroscopy. In some cases, the organic products were also isolated by chromatography and identified by NMR and mass spectroscopies ${ }^{c}$ The products $\mathbf{7}_{n_{1}}$ are phosphines $\mathrm{R}_{2} \mathrm{P}\left(\mathrm{CH}_{2} \mathrm{CH}(\mathrm{X})\right)_{n} \mathrm{CH}_{2} \mathrm{CH}_{2} \mathrm{X}$, where $\mathrm{X}=\mathrm{CO}_{2} t-\mathrm{Bu}$ or $\mathrm{CN}$ and $\mathrm{n}=1,2$, or more. ${ }^{\mathrm{n}_{\mathrm{d}}}$ up to $0{ }^{\circ} \mathrm{C}{ }^{\mathrm{e}}$ Formed after addition of excess acrylate at room temperature ${ }^{\mathrm{f}} 10$ equiv of acrylate ${ }^{\mathrm{g}}$ Formed after several days ${ }^{\mathrm{h}} 10$ equiv of acrylate plus 1 equiv of $\mathrm{H}_{2} \mathrm{O}{ }^{\mathrm{i}}$ additional unidentified products were also formed (see the experimental section/Supporting Information for details) ${ }^{\mathrm{j}} 5$ equiv of acrylate plus excess $t$ - $\mathrm{BuOH}^{\mathrm{k}}$ With 5 equiv of acrylonitrile, 
after 1 day ${ }^{1} 4.3$ equiv of acrylonitrile was added in portions ${ }^{\mathrm{m}} 10$ equiv of acrylonitrile ${ }^{\mathrm{n}} 10$ equiv of acrylonitrile plus 1 equiv of $\mathrm{H}_{2} \mathrm{O}^{\circ} 10$ equiv of acrylate ${ }^{\mathrm{p}} 1$, followed by 4 equiv of acrylate ${ }^{\mathrm{q}}$ up to room temperature ${ }^{\mathrm{r}} 1$, followed by 2 and 7 equiv of acrylate ${ }^{\mathrm{s}} 2.5$ equiv of acrylate ${ }^{\mathrm{t}} 3.5$, then 3.5 more equiv of acrylate ${ }^{u}$ No reaction below $-20^{\circ} \mathrm{C}$

\section{Reaction of $\mathrm{Pt}(\mathrm{dppe})(\mathrm{Me})\left(\mathrm{PPh}_{2}\right)$ (14) with 10 equiv of $t$-butyl acrylate at room} temperature (Entry 2, Table S1). A solution of $\mathrm{Pt}(\mathrm{dppe})(\mathrm{Me})\left(\mathrm{PPh}_{2}\right)(\mathbf{1 4}, 40 \mathrm{mg}, 0.05 \mathrm{mmol})$ in toluene- $\mathrm{d}_{8}(0.5 \mathrm{~mL})$ was transferred into an NMR tube, which was fitted with a septum. $t$-Butyl acrylate $(72 \mu \mathrm{L}, 64 \mathrm{mg}, 0.5 \mathrm{mmol})$ was added with a microliter syringe, and the reaction was monitored by ${ }^{31} \mathrm{P}$ and ${ }^{1} \mathrm{H}$ NMR spectroscopy at room temperature. After 30 minutes, no starting Ptphosphido 14 was observed. Pt complexes $\mathbf{C}$ and $\mathbf{B}$ (2 diastereomers $\mathbf{a}$ and $\mathbf{b}$ ), along with phosphines 3a and 7a1, were the only species observed in the mixture (ratio $\mathbf{3 a}: \mathbf{B}_{\mathbf{a}}: \mathbf{B}_{\mathbf{b}}: \mathbf{7} \mathbf{a} \mathbf{1}=$ 1.3:2.9:5.1:1, from integration of the $\mathrm{PPh}_{2}$ signals; ratio $\mathbf{C}: \mathbf{B}_{\mathbf{a}}: \mathbf{B}_{\mathbf{b}}=1.1: 1: 1.8$, from integration of the Duphos signals). ${ }^{1}$

After 1 day, $\mathbf{C}$ and 7a1 became the major components of the mixture. Complex $\mathbf{B}$ was still observed (ratio $\mathbf{C}: \mathbf{B}_{\mathbf{a}}: \mathbf{B}_{\mathbf{b}}=5.4: 1: 1.9$ ), whereas the phosphine 3a disappeared. The ratio $\mathbf{B}_{\mathbf{a}}: \mathbf{B}_{\mathbf{b}}: \mathbf{7 a 1}$ $=1: 1.8: 4.3$. Also, a new peak at $\delta-19.8$ was observed, whose intensity increased over the next 7 days, when a new small peak was also observed at $\delta$-18.7. These peaks, whose intensity increased over time, are assigned to phosphines 7a2 based on additional NMR and mass spectral evidence (see below). ${ }^{2}$ The ratio $\mathbf{B}_{\mathbf{a}}: \mathbf{B}_{\mathbf{b}}=1: 1.8$ did not change, but the peaks slowly disappeared. In the ${ }^{1} \mathrm{H}$ NMR spectrum peaks due to alkene $\mathbf{1 6}$ increased in intensity. After 1 month, compound $\mathbf{C}$ and phosphine 7a1 were the main components of the mixture, along with the two diastereomers of 7a2, a small amount of 3a, and a small amount of the starting Pt-phosphido 14. Alkene $\mathbf{1 6}$ was the only vinylic species observed in the ${ }^{1} \mathrm{H}$ NMR spectrum.

The mixture of phosphines was separated chromatographically. The solvent was removed under vacuum and a 9:1 petroleum ether:THF mixture $(0.5 \mathrm{~mL})$ was added to the residue, then used to elute the organic products on a silica column $(5 \mathrm{~cm}$ height, $0.6 \mathrm{~cm}$ diameter); the Pt species did not elute. The solvent was removed under vacuum to give a colorless oil. 
LRMS of the oil showed major peaks due to oxidized phosphines at $\mathrm{m} / \mathrm{z}=587.3(\mathbf{7 a 2}-\mathrm{O})$ and 459.3 (7a1-O), as well as fragmentation products which were observed in the LRMS of independently synthesized 7a1. ${ }^{2}{ }^{31} \mathrm{P},{ }^{1} \mathrm{H}$, and ${ }^{13} \mathrm{C}$ NMR $\left(\mathrm{C}_{6} \mathrm{D}_{6}\right)$ spectra of the oil confirmed it consisted mostly of $\mathbf{7 a 1}$ and $\mathbf{1 6}$, but it also contained phosphines $\mathbf{7 a 2}$ (whose ${ }^{31} \mathrm{P}$ NMR chemical shifts in $\mathrm{C}_{6} \mathrm{D}_{6}$ were the same as those reported previously in that solvent; see Table $\mathrm{S} 2$ below for ${ }^{31} \mathrm{P}$ NMR data for these and other phosphines in different solvents). ${ }^{2}$

Reaction of $\mathrm{Pt}(\mathrm{dppe})(\mathrm{Me})\left(\mathrm{PPh}_{2}\right)(14)$ with 5 equiv of $t$-butyl acrylate in 1:1 toluene/tBuOH (Entry 4, Table S1). $t$-Butanol $(0.25 \mathrm{~mL})$ was mixed with a solution of $\mathrm{Pt}$ (dppe) $(\mathrm{Me})\left(\mathrm{PPh}_{2}\right)(40 \mathrm{mg}, 0.05 \mathrm{mmol})$ in toluene $(0.25 \mathrm{~mL})$, and the mixture was transferred into an NMR tube, which was fitted with a septum. $t$-Butyl acrylate $(36 \mu \mathrm{L}, 32 \mathrm{mg}, 0.25 \mathrm{mmol})$ was added with a microliter syringe, and the reaction was monitored by ${ }^{31} \mathrm{P}$ NMR spectroscopy at room temperature.

Ten minutes after addition of $t$-butyl acrylate, the $\mathrm{PPh}_{2}$ peaks due to starting material 14 disappeared; signals of the major products $\mathrm{PPh}_{2} \mathrm{CH}_{2} \mathrm{CH}_{2} \mathrm{CO}_{2} t-\mathrm{Bu}$ ( 3 a ) and $\mathrm{PPh}_{2}\left(\mathrm{CH}_{2} \mathrm{CH}\left(\mathrm{CH}_{2} \mathrm{CH}_{2} \mathrm{CO}_{2} t-\mathrm{Bu}\right)\left(\mathrm{CO}_{2} t-\mathrm{Bu}\right)\right)$ (7a1), and of the minor products 7a2 were observed, along with unidentified signals at $\delta-13.5$ and -17.8 . After one day, the cation $\left[(\operatorname{Pt}(\mathrm{dppe})(\mathrm{Me}))_{2}(\mu-\right.$ $\left.\left.\mathrm{PPh}_{2}\right)\right]^{+}(\mathbf{1 7 a})$ was the major Pt-containing species. ${ }^{3}$ Phosphines 3 and $\mathbf{7 a} \mathbf{1}$ almost disappeared, whereas phosphines 7a2 (ratio 1:1) were the major $\mathrm{PPh}_{2}$-containing species.

Reaction of $\operatorname{Pt}(\mathrm{dppe})(\mathrm{Me})\left(\mathrm{PPh}_{2}\right)$ (14) with 1 , then 4 more equiv of acrylonitrile (Entry 5, Table S1). A solution of $\mathrm{Pt}(\mathrm{dppe})(\mathrm{Me})\left(\mathrm{PPh}_{2}\right)(40 \mathrm{mg}, 0.05 \mathrm{mmol})$ in toluene- $\mathrm{d}_{8}(0.5 \mathrm{~mL})$ was transferred into an NMR tube, which was fitted with a septum. Acrylonitrile $(3.5 \mu \mathrm{L}, 2.6 \mathrm{mg}, 0.05$ mmol) was added with a microliter syringe, and the reaction was monitored over time by ${ }^{31} \mathrm{P}$ and ${ }^{1} \mathrm{H}$ NMR spectroscopy.

After 30 min, very little unreacted starting material remained. Intermediate A (Table 2) was the major Pt-containing species, and $\mathbf{B}$ and phosphine 3a' were also observed. Acrylonitrile (4 equiv) was added. After $30 \mathrm{~min}$, the mixture contained $\mathbf{A}$, dinuclear cation $\mathbf{1 7} \mathbf{a}$, an unidentified peak at $\delta$ $-11.7,{ }^{3}$ phosphine 3a' and the two diastereomers of $\mathbf{B}$. After 1 day, A was no longer observed, and 
phosphine 3a' and the diastereomers of $\mathbf{B}(\sim 1: 1$ ratio $)$ were the major components; a small amount of phosphine 7a1' was also detected. ${ }^{1}$

Reaction of $\mathrm{Pt}(\mathrm{dppe})(\mathrm{Me})\left(\mathrm{PPh}_{2}\right)$ (14) with 1 , then $0.3,1$ and 2 more equiv of acrylonitrile (Entry 6, Table S1). A solution of $\mathrm{Pt}(\mathrm{dppe})(\mathrm{Me})\left(\mathrm{PPh}_{2}\right)(79.5 \mathrm{mg}, 0.1 \mathrm{mmol})$ in toluene- $\mathrm{d}_{8}(0.5 \mathrm{~mL})$ was transferred into an NMR tube, which was fitted with a septum. Acrylonitrile $(6.5 \mu \mathrm{L}, 5.3 \mathrm{mg}, 0.1 \mathrm{mmol})$ was added with a microliter syringe, and the reaction was monitored over time by ${ }^{31} \mathrm{P}$ and ${ }^{1} \mathrm{H}$ NMR spectroscopy.

After 1 h, unreacted phosphido complex 14 and Pt-dialkyl $\mathbf{A}$ were observed in a ratio 1:1.7, along with a small amount of the two diastereomers of B. Acrylonitrile ( 0.3 equiv) was added. The starting $\mathbf{1 4}$ and $\mathbf{A}$ were still major components of the mixture, but more compound $\mathbf{B}$ formed, and new peaks were also observed. Among these, phosphine 3a' (intense peak) and the dinuclear cation $\left[(\mathrm{Pt}(\mathrm{dppe})(\mathrm{Me}))_{2}\left(\mu-\mathrm{PPh}_{2}\right)\right]^{+}(\mathbf{1 7 a})$ could be identified. ${ }^{3}$

After $\sim 5$ h, more acrylonitrile (1 equiv) was added. Both 14 and $\mathbf{A}$ were still observed, but the major components of the mixture became the diastereomers of $\mathbf{B}$ and phosphine 3a'. Over time ( 3 h), phosphido complex 14 disappeared, signals due to $\mathbf{A}$ decreased in intensity and phosphine 7a1' formed. After 2 days, the starting phosphido could be observed again, along with small amounts of $\mathbf{A}$, the major components of the mixture being phosphine $\mathbf{3} \mathbf{a}$ and the diastereomers of B. Acrylonitrile (2 equiv) was added. After 1 day, the major species in the mixture were $\mathbf{B}$ and phosphines 3a' and 7a1'. A small amount of Pt complex $\mathbf{C}$ and dinuclear cation 17a were also observed. ${ }^{3}$

After one week, the mixture of phosphines was separated chromatographically. The solvent was removed under vacuum and a 7:3 petroleum ether:THF mixture $(0.5 \mathrm{~mL})$ was added to the residue. The Pt species were removed from the reaction mixture on a silica column $(5 \mathrm{~cm}$ height, $0.6 \mathrm{~cm}$ diameter), using a 7:3 petroleum ether:THF mixture as eluent. The Pt species did not elute. The solvent was removed under vacuum and a colorless liquid was obtained. HRMS and multinuclear NMR spectroscopy showed that the major component of the oil was phosphine $\mathbf{3 a}{ }^{2}{ }^{2} \mathrm{HRMS} \mathrm{m} / \mathrm{z}$ calcd. for $\mathrm{C}_{15} \mathrm{H}_{15} \mathrm{NP}^{+}\left(\mathrm{MH}^{+}\right)$, 240.0942, Found, 240.0948. 
Reaction of $\mathrm{Pt}(\mathrm{dppe})(\mathrm{Me})\left(\mathrm{PPh}_{2}\right)(14)$ with 10 equiv of acrylonitrile (Entry 7, Table S1). A suspension of $\mathrm{Pt}(\mathrm{dppe})(\mathrm{Me})\left(\mathrm{PPh}_{2}\right)(79 \mathrm{mg}, 0.1 \mathrm{mmol})$ in toluene $(0.5 \mathrm{~mL})$ was transferred into an NMR tube fitted with a septum. Acrylonitrile $(53 \mathrm{mg}, 66 \mu \mathrm{L}, 1 \mathrm{mmol})$ was added with a microliter syringe. The mixture was monitored by ${ }^{31} \mathrm{P}$ NMR spectroscopy. After $10 \mathrm{~min}$, the mixture became viscous and contained a pale yellow precipitate. No unreacted $\operatorname{Pt}($ dppe $)(\mathrm{Me})\left(\mathrm{PPh}_{2}\right)$ was observed. The major component of the mixture was $\mathrm{Pt}$ (dppe) $(\mathrm{Me})\left(\mathrm{CH}(\mathrm{CN}) \mathrm{CH}_{2} \mathrm{CH}(\mathrm{CN}) \mathrm{CH}_{2} \mathrm{PPh}_{2}\right)(\mathbf{B}$, as a 1:1 mixture of diastereomers). Small amounts of $\mathrm{Pt}(\mathrm{dppe})(\mathrm{Me})\left(\mathrm{CH}(\mathrm{CN}) \mathrm{CH}_{2} \mathrm{PPh}_{2}\right) \quad$ (A) and phosphines 3a' and 7a1' were also observed. After two days, red precipitate formed on the walls of the NMR tube. No compound A was observed, and the mixture consisted of $\mathbf{B}$ plus phosphines 3a' and a small amount of 7a1'. ${ }^{1}$

Reaction of $\mathrm{Pt}(\mathrm{dppe})(\mathrm{Me})\left(\mathrm{PPh}_{2}\right)(14)$ with 10 equiv of acrylonitrile in the presence of 1 equiv of $\mathrm{H}_{2} \mathrm{O}$ (Entry 8, Table S1). A suspension of $\mathrm{Pt}(\mathrm{dppe})(\mathrm{Me})\left(\mathrm{PPh}_{2}\right)(79 \mathrm{mg}, 0.1 \mathrm{mmol})$ in toluene $(0.5 \mathrm{~mL})$ was transferred into an NMR tube fitted with a septum. $\mathrm{H}_{2} \mathrm{O}(2 \mathrm{mg}, 2 \mu \mathrm{L}, 0.1$ mmol) was added with a microliter syringe, followed by acrylonitrile $(53 \mathrm{mg}, 66 \mu \mathrm{L}, 1 \mathrm{mmol})$. The mixture was monitored by ${ }^{31} \mathrm{P}$ NMR spectroscopy. After 30 min, no unreacted $\mathrm{Pt}(\mathrm{dppe})(\mathrm{Me})\left(\mathrm{PPh}_{2}\right)$ was observed. The main components of the mixture were phosphine $\mathbf{3 a}$ ' and $\mathbf{B}$, as a 1:1 mixture of diastereomers. Small amounts of $\mathbf{A}$ and 7a1' were also observed. After 3 days, much red precipitate had formed on the walls of the NMR tube. The only $\mathrm{PPh}_{2}$-containing species observed were phosphines 3a' (major) and 7a1', along with very small amounts of $\mathbf{B}$ and 7an'. ${ }^{1}$ In the $\mathrm{Pt}($ dppe $)$ region, two unidentified Pt compounds were observed: $\left(\delta 49.0\left(\mathrm{~J}_{\mathrm{Pt}-\mathrm{P}}=1774\right), 47.8\left(\mathrm{~J}_{\mathrm{Pt}-\mathrm{P}}=\right.\right.$ $2312)$ and $\left.\left.\delta 48.7\left(\mathrm{~J}_{\mathrm{Pt}-\mathrm{P}}=2828\right), 45.7\right)\right)$. A small amount of the cation $\left[(\mathrm{Pt}(\mathrm{dppe})(\mathrm{Me}))_{2}\left(\mu-\mathrm{PPh}_{2}\right)\right]^{+}$

(17a) was also observed. After 2 weeks, B had disappeared and an additional unidentified, symmetrical Pt species $\left(\delta 31.9\left(\mathrm{~J}_{\mathrm{Pt}-\mathrm{P}}=3730\right)\right.$, probably $\left.\mathrm{Pt}(\mathrm{dppe})_{2}\right)$ was also detected. ${ }^{4}$

Reaction of $\operatorname{Pt}(\mathrm{dppe})(\mathrm{Me})(\mathrm{PPh}(i-\mathrm{Bu}))(15)$ with 10 equiv of $t$-butyl acrylate at room temperature (Entry 9, Table S1). A solution of $\mathrm{Pt}(\mathrm{dppe})(\mathrm{Me})(\mathrm{PPh}(i-\mathrm{Bu}))(39 \mathrm{mg}, 0.05 \mathrm{mmol})$ in toluene- $\mathrm{d}_{8}(0.5 \mathrm{~mL})$ was transferred into an NMR tube, which was fitted with a septum. $t$-Butyl 
acrylate $(72 \mu \mathrm{L}, 64 \mathrm{mg}, 0.5 \mathrm{mmol})$ was added with a microliter syringe, and the reaction was monitored by ${ }^{31} \mathrm{P}$ and ${ }^{1} \mathrm{H}$ NMR spectroscopy at room temperature.

After $1 \mathrm{~h}$, complex 15 had been consumed; the main components of the mixture were phosphines $\mathbf{3 b}$ and the two diastereomers (ratio 1:1) of $\mathbf{7 b 1} .^{2}$ Six more peaks, which showed no $\mathrm{J}_{\mathrm{Pt}-\mathrm{P}}$ coupling, with intensities comparable to those of phosphines $\mathbf{3 b}$ and $\mathbf{7 b \mathbf { b }}$, could also be observed in the $\operatorname{PPh}(i-\mathrm{Bu})$ region; four could be assigned to diastereomers of $\mathbf{B}$. In the dppe region, peaks due to the four possible diastereomers of $\mathbf{B}$ (ratio 11.8:5.1:1.8:1; Table 3) and to $\mathbf{C}$ (Table 4) were observed. The ratio C:B was about 1.1:1 (based on integration of the dppe peaks). Unexpectedly, a small amount of a symmetrical Pt(dppe) species $\left(\delta 47.8, \mathrm{~J}_{\mathrm{Pt}-\mathrm{P}}=1779\right)$, perhaps $\mathrm{Pt}(\mathrm{dppe}) \mathrm{Me}_{2}$, was also observed. ${ }^{5}$ The ${ }^{1} \mathrm{H}$ NMR spectrum showed the presence of unreacted $t$-butyl acrylate. After 1 day, the main components of the mixture were $\mathbf{C}$, phosphines $\mathbf{7 b 1}$ (ratio 1:1) and $\mathbf{3 b}$, along with decreasing amounts of $\mathbf{B}$. The other two unidentified peaks in the $\mathrm{PPh}(i-\mathrm{Bu})$ region $(\delta-32.7,-32.9)$ had increased in intensity. "Pt(dppe)Me ${ }_{2}$ " was also observed. ${ }^{5}$

After 8 days, the main species were $\mathbf{C}$ and "Pt(dppe)Me ${ }_{2}$ ", the diastereomeric phosphines $\mathbf{7 b} \mathbf{1}$ (ratio 1:1), and the $\delta-32.7$ and -32.9 materials. Other $\mathrm{PPh}(i-\mathrm{Bu})$ signals, two of them in a relatively big amount $(\delta-31.7,-32.3$, ratio $\sim 1: 1)$, were also detected, and assigned as $\mathbf{7 b 2}$. As previously seen in decomposition of $\mathbf{1 5}$, the dinuclear cation $\left[(\operatorname{Pt}(\mathrm{dppe})(\mathrm{Me}))_{2}(\mu-\mathrm{PPh}(i-\mathrm{Bu}))\right]^{+}(\mathbf{1 7} \mathbf{b})$ and unidentified species with signals at $\delta 31.7,29.8,-11.8$ were also observed. ${ }^{3}$ More mysteriously, a Pt-phosphido species appeared $\left({ }^{31} \mathrm{P}\right.$ NMR $\left(\right.$ toluene- $\left.\mathrm{d}_{8}\right): \delta 47.8\left(\mathrm{~d}, \mathrm{~J}=143, \mathrm{~J}_{\mathrm{Pt}-\mathrm{P}}=1923\right), 44.7(\mathrm{~d}, \mathrm{~J}$ $\left.=18, \mathrm{~J}_{\mathrm{Pt}-\mathrm{P}}=1825\right),-8.8\left(\mathrm{dd}, \mathrm{J}=143,18, \mathrm{~J}_{\mathrm{Pt}-\mathrm{P}}=1040\right)$; this spectrum is essentially identical to that of phosphido complex 14, but it is not clear how that could be formed under these conditions) ${ }^{6}$ and another peak $(\delta-19.0)$ in a ratio 1:1 with the peak at -11.8 , was observed. The ${ }^{1} \mathrm{H}$ NMR spectrum of the mixture showed alkene $\mathbf{1 6}$ as the only vinyl species.

After 9 days, the mixture of phosphines was separated chromatographically. The solvent was removed under vacuum and a 9:1 petroleum ether:THF mixture $(0.5 \mathrm{~mL})$ was added to the residue. The Pt species were removed from the reaction mixture on a silica column $(5 \mathrm{~cm}$ height, $0.6 \mathrm{~cm}$ 
diameter), using a 9:1 petroleum ether:THF mixture as eluent. The Pt species did not elute. The solvent was removed under vacuum and a colorless oil was obtained.

LRMS of the oil showed major peaks at $\mathrm{m} / \mathrm{z}=695.5(\mathbf{7 b 3 - O}), 567.4(\mathbf{7 b 2}-\mathrm{O})$ and $439.3(\mathbf{7 b 1 -}$ O), as well as fragmentation products previously observed for $\mathbf{7 b 1}:^{2} \mathrm{~m} / \mathrm{z}=399.2(\mathbf{7 b 2}-\mathrm{O}, \mathrm{n}=2$, after loss of $\left.3 \mathrm{Me}_{2} \mathrm{C}=\mathrm{CH}_{2}\right)$ and $327.2\left(7 \mathbf{b} 1-\mathrm{O}, \mathrm{n}=1\right.$, after loss of $\left.2 \mathrm{Me}_{2} \mathrm{C}=\mathrm{CH}_{2}\right)$. The peaks at 439.3 (7b1-O, $\mathrm{n}=1)$ and $327.1\left(\mathbf{7 b 1}-\mathrm{O}, \mathrm{n}=1\right.$, after loss of $\left.2 \mathrm{Me}_{2} \mathrm{C}=\mathrm{CH}_{2}\right)$ had high intensities in

the LRMS of 7b1. ${ }^{2}{ }^{31} \mathrm{P},{ }^{1} \mathrm{H}$, and ${ }^{13} \mathrm{C}$ NMR $\left(\mathrm{C}_{6} \mathrm{D}_{6}\right)$ spectra of the separated colorless liquid confirmed it consisted mostly of $\mathrm{PPh}(i-\mathrm{Bu})\left(\mathrm{CH}_{2} \mathrm{CH}\left(\mathrm{CH}_{2} \mathrm{CH}_{2} \mathrm{CO}_{2} t \mathrm{Bu}\right)\left(\mathrm{CO}_{2} t \mathrm{Bu}\right)\right)(7 \mathbf{b 1})$ and alkene 16, but it also contained phosphines $7 \mathbf{b 2}$, whose ${ }^{31} \mathrm{P}$ NMR chemical shifts in $\mathrm{C}_{6} \mathrm{D}_{6}$ were the same as those previously reported (Table S2). ${ }^{1,2}$

Reaction of $\operatorname{Pt}(\operatorname{dppe})(\mathrm{Me})(\operatorname{PPh}(i$-Bu) $)(15)$ with 1 equiv of $t$-butyl acrylate at room temperature followed by addition of 4 equiv of $t$-butyl acrylate (Entry 10, Table S1). A solution of $\mathrm{Pt}(\mathrm{dppe})(\mathrm{Me})(\mathrm{PPh}(i-\mathrm{Bu}))(39 \mathrm{mg}, 0.05 \mathrm{mmol})$ in toluene- $\mathrm{d}_{8}(0.5 \mathrm{~mL})$ was transferred into an NMR tube, which was fitted with a septum. $t$-Butyl acrylate $(7 \mu \mathrm{L}, 6.4 \mathrm{mg}, 0.05 \mathrm{mmol})$ was added with a microliter syringe, and the reaction was monitored by ${ }^{31} \mathrm{P}$ and ${ }^{1} \mathrm{H}$ NMR spectroscopy at room temperature. After $10 \mathrm{~min}$, most of $\mathbf{1 5}$ had reacted, and the main $\mathrm{PPh}(i$-Bu) peaks were due to the four diastereomers of $\mathbf{B}$. Very little of the phosphines $\mathbf{3 b}$ and $\mathbf{7 b} \mathbf{b}$ were observed. ${ }^{1}$

After 1 day, the signals due to the starting $\mathbf{1 5}$ and the phosphines $\mathbf{7 b 1}$ increased, while those of $\mathbf{B}$ decreased in intensity. The other phosphido complex (see above for experimental details of the reaction in entry 9 , Table $\mathrm{S} 1$ ), the dinuclear cation $\mathbf{1 7 b}$, along with additional peaks at $\delta 31.6,29.6$, and -11.9 were also present, but no Pt-dialkyl $\mathbf{C}$ could be observed. Alkene 16 was observed by ${ }^{1} \mathrm{H}$ NMR (toluene- $\left.d_{8}\right)$.

After 3 days, $t$-butyl acrylate (4 equiv) was added. After 5 minutes, complex $\mathbf{B}$ was the main component of the mixture, along with phosphines $\mathbf{3 b}$ (very little), $\mathbf{7 b 1}$ and the two species at $\delta-32.7$ and -32.9. Other compounds present included " $\mathrm{Pt}(\mathrm{dppe}) \mathrm{Me}_{2}{ }^{\prime}{ }^{5}$ along with some $\mathrm{PR}_{2}$ peaks which don't seem to be $\mathrm{PPh}(i-\mathrm{Bu})$ species: $\delta$-9.7, $-11.8,-17.1,-17.8,-18.2,-18.4,-19.0$. 


\section{Reaction of $\operatorname{Pt}(\operatorname{dppe})(\mathrm{Me})(\mathrm{PPh}(i-\mathrm{Bu}))(15)$ with $t$-butyl acrylate in 1:1 toluene/t-BuOH}

(Entry 11, Table S1). $t$-Butanol $(0.25 \mathrm{~mL})$ was mixed with a solution of $\operatorname{Pt}($ dppe $)(\mathrm{Me})(\operatorname{PPh}(i$ $\mathrm{Bu})$ ) (39 $\mathrm{mg}, 0.05 \mathrm{mmol})$ in toluene $(0.25 \mathrm{~mL})$, and the mixture was transferred into an NMR tube, which was fitted with a septum. $t$-Butyl acrylate $(36 \mu \mathrm{L}, 32 \mathrm{mg}, 0.25 \mathrm{mmol})$ was added with a microliter syringe, and the reaction was monitored by ${ }^{31} \mathrm{P}$ NMR spectroscopy at room temperature.

Ten minutes after addition of $t$-butyl acrylate, the starting phosphido complex $\mathbf{1 5}$ and the cationic dinuclear compound $\left[(\operatorname{Pt}(\mathrm{dppe})(\mathrm{Me}))_{2}(\mu-\mathrm{PPh}(i-\mathrm{Bu}))\right]^{+}(\mathbf{1 7 b})$ were the major components of the mixture. ${ }^{3}$ Four other $\operatorname{PPh}(i-\mathrm{Bu})$ peaks were also observed. The two major ones $(\delta-31.8,-32.1$, about 1:1) might be assigned to the two diastereomers of $\mathrm{PPh}(i-\mathrm{Bu})\left(\mathrm{CH}_{2} \mathrm{CH}\left(\mathrm{CH}_{2} \mathrm{CH}_{2} \mathrm{CO}_{2} t\right.\right.$ $\left.\mathrm{Bu})\left(\mathrm{CO}_{2} t-\mathrm{Bu}\right)\right)(\mathbf{7 b 1})$. The other two peaks that also show no Pt-P coupling $(\delta-31.7,-32.8)$, can be tentatively assigned to phosphines containing more acrylate units (7bn, $n>1)$. The intensity of the peaks assigned to $\mathbf{7 b 1}$ increased over time. ${ }^{1}$

Reaction of $t$-Bu acrylate with $\operatorname{Pt}((\mathbf{R}, \mathbf{R})-\mathrm{Me}-\mathrm{Duphos})(\mathrm{Me})\left(\mathrm{PPh}_{2}\right)$ (11) at low temperature (Entry 12, Table S1). A solution of $\mathrm{Pt}((\mathrm{R}, \mathrm{R})-\mathrm{Me}-\mathrm{Duphos})(\mathrm{Me})\left(\mathrm{PPh}_{2}\right)(\mathbf{1 1}, 34 \mathrm{mg}$, $0.05 \mathrm{mmol})$ in toluene- $\mathrm{d}_{8}(0.5 \mathrm{~mL})$ was transferred into an NMR tube, which was fitted with a septum. The NMR tube was cooled to $-50{ }^{\circ} \mathrm{C}$ and $t$-butyl acrylate $(7 \mu \mathrm{L}, 6.4 \mathrm{mg}, 0.05 \mathrm{mmol})$ was added with a microliter syringe. The tube was immediately inserted in the NMR spectrometer, which was precooled to $-50{ }^{\circ} \mathrm{C}$, and the reaction was monitored by ${ }^{31} \mathrm{P}$ and ${ }^{1} \mathrm{H}$ NMR spectroscopy, from $-75^{\circ} \mathrm{C}$ to room temperature.

Intermediate A (2:1 mixture of diastereomers $\mathbf{a}$ and $\mathbf{b}$, Table 2$)$, was observed immediately after addition of the acrylate at $-50{ }^{\circ} \mathrm{C}$. Note that at $-50{ }^{\circ} \mathrm{C}$, peaks due to $\mathbf{b}$ were broad, but those of $\mathbf{a}$ were sharp. A was the major component of the mixture, along with the starting 11, but it disappeared slowly on warming; A signals were still observed at $21{ }^{\circ} \mathrm{C}, 4 \mathrm{~h}$ after the addition of acrylate. ${ }^{31} \mathrm{P}\left\{{ }^{1} \mathrm{H}\right\}$ NMR data (toluene- $\mathrm{d}_{8},-75^{\circ} \mathrm{C}$ ) for intermediate $\mathbf{A}$ : for the major isomer $\mathbf{a}, \delta 65.5$ $\left(\mathrm{d}, \mathrm{J}=34, \mathrm{~J}_{\mathrm{Pt}-\mathrm{P}}=2149\right), 67.9\left(\mathrm{~J}_{\mathrm{Pt}-\mathrm{P}}=1775\right) ;-11.6\left(\mathrm{~d}, \mathrm{~J}=34, \mathrm{~J}_{\mathrm{Pt}-\mathrm{P}}=265\right)$. For minor isomer $\mathbf{b}$, $\delta 65.9\left(\mathrm{~d}, \mathrm{~J}=34, \mathrm{~J}_{\mathrm{Pt}-\mathrm{P}}=2145\right), 65.5\left(\mathrm{~J}_{\mathrm{Pt}-\mathrm{P}}=1793\right),-10.5\left(\mathrm{~d}, \mathrm{~J}=34, \mathrm{~J}_{\mathrm{Pt}-\mathrm{P}}=296\right)$. 
A very small amount of $\mathrm{PPh}_{2} \mathrm{CH}_{2} \mathrm{CH}_{2} \mathrm{CO}_{2} t-\mathrm{Bu}$ (3 a), and much more $\mathrm{PPh}_{2}\left(\mathrm{CH}_{2} \mathrm{CH}\left(\mathrm{CH}_{2} \mathrm{CH}_{2} \mathrm{CO}_{2} t-\mathrm{Bu}\right)\left(\mathrm{CO}_{2} t-\mathrm{Bu}\right)\right)$ (7a1) were observed immediately after addition of the acrylate at $-50{ }^{\circ} \mathrm{C}$, in an approximate ratio $\mathbf{3 a : 7 a 1}=1: 10{ }^{2}$ The ratio varied little on warming up the reaction mixture to room temperature (over $\sim 4 \mathrm{~h}$ ). One of the four possible diastereomers of Ptdialkyl product B-11 was also observed immediately after the addition of the acrylate. A small amount of the second diastereomer was observed starting at $-60{ }^{\circ} \mathrm{C}$; then, at $-20{ }^{\circ} \mathrm{C}$ and $0{ }^{\circ} \mathrm{C}$, the third and fourth diastereomers were observed. The ratio between the four diastereomers changed with temperature, the one that was first observed at $-60{ }^{\circ} \mathrm{C}$ becoming major at room temperature ( 3:2:1:1 a-d, Table 3). The starting compound 11 was still the major component of the mixture when it reached room temperature. Unreacted $t$-butyl acrylate was the only vinyl species observed in the ${ }^{1} \mathrm{H}$ NMR spectrum, even at room temperature.

Reaction of $\operatorname{Pt}((\mathrm{R}, \mathrm{R})-\mathrm{Me}-\mathrm{Duphos})(\mathrm{Me})\left(\mathrm{PPh}_{2}\right)$ with 1 equiv of t-butyl acrylate at room temperature (Entry 13, Table S1). A solution of $\mathrm{Pt}\left((\mathrm{R}, \mathrm{R}-\mathrm{Me}-\mathrm{Duphos})(\mathrm{Me})\left(\mathrm{PPh}_{2}\right)(35 \mathrm{mg}, 0.05\right.$ mmol $)$ in $\mathrm{C}_{6} \mathrm{D}_{6}(0.5 \mathrm{~mL})$ was transferred into an NMR tube, which was fitted with a septum. $t$-Butyl acrylate $(7 \mu \mathrm{L}, 6.4 \mathrm{mg}, 0.05 \mathrm{mmol})$ was added with a microliter syringe. The reaction was monitored over time by ${ }^{31} \mathrm{P}$ and ${ }^{1} \mathrm{H}$ NMR spectroscopy, at room temperature.

After $15 \mathrm{~h}$, the amount of starting $\mathbf{1 1}$ decreased, and Pt-dialkyl compound $\mathbf{A}$ was not detected. Phosphines 3a and 7a1 (approximate ratio 1:3), along with the four diastereomers of the Pt-dialkyl compound B were observed. ${ }^{1}$ Peak overlap in the Duphos region precluded identification of $\mathbf{C}$. After 1 week, the peaks of starting $\mathbf{1 1}$ decreased, phosphine 3a almost disappeared, and the amount of phosphine 7a1 increased; the ratio of the four diastereomers of $\mathbf{B}$ varied over time. In the ${ }^{1} \mathrm{H}$ NMR spectrum, unreacted $t$-butyl acrylate and its dimer, 16, were observed.

Reaction of $\operatorname{Pt}((\mathrm{R}, \mathrm{R})-\mathrm{Me}-\mathrm{Duphos})(\mathrm{Me})\left(\mathrm{PPh}_{2}\right)$ with 1 equiv of t-butyl acrylate at room temperature, followed by subsequent addition of 2, then 7 equiv of t-butyl acrylate (Entry 14, Table S1). A solution of $\mathrm{Pt}((\mathrm{R}, \mathrm{R})-\mathrm{Me}-\mathrm{Duphos})(\mathrm{Me})\left(\mathrm{PPh}_{2}\right)(35 \mathrm{mg}, 0.05 \mathrm{mmol})$ in $\mathrm{C}_{6} \mathrm{D}_{6}(0.5$ $\mathrm{mL}$ ) was transferred into an NMR tube, which was fitted with a septum. $t$-Butyl acrylate (7 $\mu \mathrm{L}, 6.4$ 
$\mathrm{mg}, 0.05 \mathrm{mmol}$ ) was added with a microliter syringe. The reaction was monitored over time by ${ }^{31} \mathrm{P}$ and ${ }^{1} \mathrm{H}$ NMR spectrometry, at room temperature.

After $1 \mathrm{~h}$, the starting Pt-phosphido 11 was the main component of the mixture, and no compound $\mathbf{A}$ could be detected. Small amounts of phosphines 3a and 7a1 (approximate ratio = 5:1) were observed, along with one of the four diastereomers of the Pt-dialkyl compound $\mathbf{B}(\delta$ -18.1, Table 3). $t$-Butyl acrylate (2 equiv) was added. After $1 \mathrm{~h}$, the amount of phosphines 3a and 7a1 increased, and their ratio became 1:2. All four diastereomers of $\mathbf{B}$ were also observed. ${ }^{1}$

After 1 day, a small amount of the starting Pt-phosphido 11 remained, and in addition to phosphines 3a (which almost disappeared) and 7a1, the four diastereomers of $\mathbf{B}$, and $\mathbf{C}$, a new small peak was observed at $\delta$-19.9. Unreacted $t$-butyl acrylate was observed in the ${ }^{1} \mathrm{H}$ NMR spectrum. $t$-Butyl acrylate ( 3 equiv) was added to half of the above mixture. No starting $\mathbf{1 1}$ or phosphine 3a were observed. Phosphine 7a1, the four diastereomers of $\mathbf{B}$, and $\mathbf{C}$ were observed. After 4 days, 7a1 and $\mathbf{C}$ were the major components of the mixture, as $\mathbf{B}$ slowly disappeared.

Reaction of $t$-Bu acrylate with $\operatorname{Pt}((\mathrm{R}, \mathrm{R})-\mathrm{Me}-\mathrm{Duphos})(\mathrm{Me})(\mathrm{PPh}(i-\mathrm{Bu}))(12)$ at low temperature (Entry 15, Table S1). A solution of $\mathrm{Pt}((\mathrm{R}, \mathrm{R})-\mathrm{Me}-\mathrm{Duphos})(\mathrm{Me})(\mathrm{PPh}(i-\mathrm{Bu}))(34$ $\mathrm{mg}, 0.05 \mathrm{mmol})$ in toluene- $\mathrm{d}_{8}(0.5 \mathrm{~mL})$ was transferred into an NMR tube, which was fitted with a septum. The NMR tube was cooled to $-50{ }^{\circ} \mathrm{C}$ and $t$-butyl acrylate $(7 \mu \mathrm{L}, 6.4 \mathrm{mg}, 0.05 \mathrm{mmol})$ was added with a microliter syringe. The tube was immediately inserted in the NMR spectrometer, which had been precooled to $-50{ }^{\circ} \mathrm{C}$, and the reaction was monitored by ${ }^{31} \mathrm{P} \mathrm{NMR}$ spectroscopy, from $-75^{\circ} \mathrm{C}$ to room temperature.

The Pt-dialkyl A (only two of the four possible diastereomers, $\mathbf{a}$ and $\mathbf{b}$, Table 2), was observed immediately after addition of the acrylate at $-50{ }^{\circ} \mathrm{C}$. The major component of the mixture was the starting phosphido compound 12 (1:1 mixture of diastereomers). Several ( 10) peaks which showed no Pt-P coupling, with different intensities, were observed in the $\mathrm{PPh}(i$-Bu $)$ region. At -75 ${ }^{\circ} \mathrm{C}$, the intensity of the A peaks decreased, but they could be observed up to $0{ }^{\circ} \mathrm{C}$. The starting phosphido 12 was still the major component of the mixture, and the intensities of the $\mathrm{PPh}(i-\mathrm{Bu})$ peaks also changed with temperature. At room temperature, the main Pt species, apart from the 
starting 12, was C. Also, phosphines $\mathbf{3 b}$ and $\mathbf{7 b 1}$ (ratio $\mathbf{7 b} \mathbf{1}_{\mathbf{a}}: \mathbf{7 b}_{\mathbf{b}} \sim 1: 5$ ), along with other peaks that don't show any $\mathrm{Pt}-\mathrm{P}$ coupling, could be observed in the $\mathrm{PPh}(i$-Bu $)$ region. Their chemical shifts are close to those assigned to diastereomers of $\mathbf{B}$, in the experiments done at room temperature. After 4 days, the only change observed was the different ratio $\mathbf{7 b}_{\mathbf{a}}: \mathbf{7 b}_{\mathbf{b}} \sim 1: 1$, disappearance of some of the $\mathrm{PPh}(i$-Bu $)$ peaks, and the slightly increased intensity of the $\mathbf{3 b}$ peak. ${ }^{1}$

Reaction of $\operatorname{Pt}((\mathbf{R}, \mathbf{R})-M e-D u p h o s)(M e)(\operatorname{PPh}(i-B u))(12)$ with 1 equiv of $t$-butyl acrylate at room temperature (Entry 16, Table S1). A solution of impure $\mathrm{Pt}((\mathrm{R}, \mathrm{R})-\mathrm{Me}$ Duphos $)(\mathrm{Me})(\mathrm{PPh}(i-\mathrm{Bu}))(34 \mathrm{mg}, 0.05 \mathrm{mmol})$ in $\mathrm{C}_{6} \mathrm{D}_{6}(0.5 \mathrm{~mL})$ was transferred into an NMR tube, which was fitted with a septum. $t$-Butyl acrylate $(7 \mu \mathrm{L}, 6.4 \mathrm{mg}, 0.05 \mathrm{mmol})$ was added with a microliter syringe. The reaction was monitored over time by ${ }^{31} \mathrm{P}$ and ${ }^{1} \mathrm{H}$ NMR spectroscopy, at room temperature.

After $15 \mathrm{~h}$, the amount of starting 12 decreased, and Pt-dialkyl compound $\mathbf{A}$ was not detected. Phosphines $\mathbf{3 b}$ and the diastereomers of $\mathbf{7 b 1}$ (ratio 7b1:3b $>10$, ratio $\mathbf{7 b} \mathbf{1}_{\mathbf{a}}: \mathbf{7 b}_{\mathbf{b}} \sim 3: 1, \mathbf{7 b 1}_{\mathbf{a}}: \delta$ $\left.32.0, \mathbf{7 b 1}_{\mathbf{b}}: \delta-32.5\right)$, along with seven other peaks in the $\mathrm{PPh}(i-\mathrm{Bu})$ region which lacked $\mathrm{Pt}-\mathrm{P}$ coupling, could be observed $(\delta-31.0,-31.3,-31.5,-32.2,-32.3,-32.8,-33.3) .{ }^{1}$ It is likely that these belong to the eight diastereomers of $\mathbf{B}$ (Table 3), but the Duphos region was very complicated, precluding assignment or identification of other $\mathrm{Pt}(\mathrm{Duphos})$ complexes. After 1 week, the ratio $\mathbf{7 b 1}_{\mathbf{a}}: \mathbf{7 b 1}_{\mathbf{b}}$ became $\sim 1: 1.2$, favoring the other diastereomer. The ratio between the other seven $\mathrm{PPh}(i-\mathrm{Bu})$ species changed; three of the peaks increased in intensity and now only six were observed. The dimer of $t$-butyl acrylate (16) was the main vinyl species observed in the ${ }^{1} \mathrm{H}$ NMR spectrum.

\section{Reaction of $\operatorname{Pt}((\mathrm{R}, \mathrm{R})-\mathrm{Me}-\mathrm{Duphos})(\mathrm{Me})(\operatorname{PPh}(i-\mathrm{Bu}))(12)$ with 2.5 equiv of t-butyl} acrylate at room temperature (Entry 17, Table S1). A solution of impure $\mathrm{Pt}((\mathrm{R}, \mathrm{R})-\mathrm{Me}$ Duphos)(Me)(PPh $(i-\mathrm{Bu}))(30 \mathrm{mg}, 0.044 \mathrm{mmol})$ in $\mathrm{C}_{6} \mathrm{D}_{6}(0.5 \mathrm{~mL})$ was transferred into an NMR tube, which was fitted with a septum. $t$-Butyl acrylate $(16 \mu \mathrm{L}, 14 \mathrm{mg}, 0.11 \mathrm{mmol})$ was added with a microliter syringe. The reaction was monitored over time by ${ }^{31} \mathrm{P}$ and ${ }^{1} \mathrm{H}$ NMR spectroscopy, at room temperature. 
After 1 h, no starting material 12 was observed, and Pt-dialkyl compound A was not detected. Diastereomeric phosphines $\mathbf{7 b 1}$ (ratio $\mathbf{7 b 1}_{\mathbf{a}} \mathbf{:} \mathbf{7 b 1}_{\mathbf{b}} \sim 20: 1$ ), along with eight other peaks in the $\operatorname{PPh}(i-\mathrm{Bu})$ region, the same as those observed in entry 16 above, plus another peak at $\delta-32.4)$, were observed. ${ }^{1}$

After 1 day, the ratio $\mathbf{7 b 1}_{\mathbf{a}}: \mathbf{7 b}_{\mathbf{b}}$ changed to $\sim 10: 1$. Duphos signals assigned to some of the eight diastereomers of compound $\mathbf{B}$ were identified (Table 3), and complex $\mathbf{C}$ was also observed (Table 4). After 3 days, the ratio $\mathbf{7 b} \mathbf{1}_{\mathbf{a}}: \mathbf{7 b}_{\mathbf{b}} \sim 1: 1$, and after 10 days it remained almost unchanged. Most of the peaks that were assigned to the diastereomers of $\mathbf{B}$ decreased in intensity. Also, formation of the cation $\left[(\operatorname{Pt}((\mathrm{R}, \mathrm{R})-\mathrm{Me}-\mathrm{Duphos})(\mathrm{Me}))_{2}(\mu \text {-PPh }(i-\mathrm{Bu}))\right]^{+}$was observed. ${ }^{3}$ The same observations were made when the experiment was performed in $\mathrm{THF}-\mathrm{d}_{8}$.

Reaction of $\operatorname{Pt}((\mathrm{R}, \mathrm{R})-\mathrm{Me}-\mathrm{Duphos})(\mathrm{Me})(\mathrm{PPh}(i-\mathrm{Bu}))(12)$ with 3.5 and 7 equiv of $\mathrm{t}-\mathrm{butyl}$ acrylate at room temperature (Entry 18, Table S1). A solution of impure $\mathrm{Pt}((\mathrm{R}, \mathrm{R})-\mathrm{Me}-$ Duphos)(Me)(PPh $(i-\mathrm{Bu}))(6 \mathrm{mg}, 0.008 \mathrm{mmol})$ in toluene- $\mathrm{d}_{8}(0.5 \mathrm{~mL})$ was transferred into an NMR tube, which was fitted with a septum. $t$-Butyl acrylate ( $4 \mu \mathrm{L}, 3 \mathrm{mg}, 0.03 \mathrm{mmol})$ was added

with a microliter syringe. The reaction was monitored over time by ${ }^{31} \mathrm{P}$ and ${ }^{1} \mathrm{H}$ NMR spectroscopy, at room temperature.

After 10 min, no starting phosphido compound could be detected, and the major components of the mixture were compound $\mathbf{C}$ and phosphines $\mathbf{3 b}$ and $\mathbf{7 b 1}$. Other peaks which showed no Pt-P coupling could be seen at $\delta-30.8,-31.2,-32.8$, and -33.1 . $t$-Butyl acrylate (3.5 equiv) was added. After 15 h, compound $\mathbf{C}$ was the main $\mathrm{Pt}($ Duphos) species. Phosphines $\mathbf{3 b}$ and $\mathbf{7 b \mathbf { 1 }}$ (approximate ratio $\left.\mathbf{7 b} \mathbf{1}_{\mathbf{a}}: \mathbf{7 b 1}_{\mathbf{b}}=1: 1.5\right)$ were also observed, along with the other previously mentioned $\mathrm{PPh}(i-\mathrm{Bu})$ peaks. ${ }^{1}$

Reaction of $t$-Bu acrylate with Pt((R,R)-Me-Duphos)(Ph)(PMeIs) (13) at low temperature (Entry 19, Table S1). A solution of $\mathrm{Pt}((\mathrm{R}, \mathrm{R})-\mathrm{Me}-\mathrm{Duphos})(\mathrm{Ph})(\mathrm{PMeIs})(41 \mathrm{mg}$, $0.05 \mathrm{mmol})$ in toluene- $\mathrm{d}_{8}(0.5 \mathrm{~mL})$ was transferred into an NMR tube, which was fitted with a septum. The NMR tube was cooled to $-50{ }^{\circ} \mathrm{C}$ and $t$-butyl acrylate $(7 \mu \mathrm{L}, 6.4 \mathrm{mg}, 0.05 \mathrm{mmol})$ was added with a microliter syringe. The tube was immediately inserted in the NMR spectrometer, 
which was previously cooled to $-50{ }^{\circ} \mathrm{C}$, and the reaction was monitored by ${ }^{31} \mathrm{P}$ and ${ }^{1} \mathrm{H}$ NMR spectroscopy, from $-75^{\circ} \mathrm{C}$ to room temperature.

No reaction was observed until the temperature reached $-20{ }^{\circ} \mathrm{C}$. At $0{ }^{\circ} \mathrm{C}$, three of the four possible diastereomers of $\mathbf{A}$ were observed (Table 2). The intensity of these peaks decreased upon warming the reaction mixture to room temperature, and phosphine $\mathbf{3 c}$ could also be observed. Heating the reaction mixture to $80{ }^{\circ} \mathrm{C}$ in the NMR probe caused the disappearance of $\mathbf{A}$, and formation of more $\mathbf{3 c}$, but after cooling to room temperature, $\mathbf{A}$ was observed again.

After 1 day at room temperature, more $3 \mathbf{c}$ formed, and one diastereomer of $\mathbf{7 c 1}$ appeared. ${ }^{1} t$-Bu acrylate (4 equiv) was added. The intensity of the peaks due to $\mathbf{A}$ and phosphine $\mathbf{3 c}$ increased, both diastereomers of $\mathbf{7} \mathbf{1} 1$ were observed and new peaks were detected at $\delta-44.8,-52.6$. After 1 day, the intensity of the $\mathbf{A}$ and $\mathbf{3 c}$ peaks decreased, and a few new peaks, overlapping the PMeIs signals of the starting phosphido compound 13, could be observed; the major Pt species was $\mathbf{C}$. Compound $\mathbf{A}$ and the starting $\mathbf{1 3}$ disappeared over time, as $\mathbf{C}$ formed. After 1 month, only phosphines $\mathbf{7 c 1}$ and other singlet signals, that can probably be assigned to some of the eight diastereomers of compound B, were observed in the PMeIs region. 
Table S2. ${ }^{31} \mathrm{P}$ NMR chemical shifts in different solvents for independently synthesized phosphines $^{2}$

$$
\overbrace{3}^{P_{3}} \overbrace{x} \overbrace{n}^{P R_{2}}
$$

\begin{tabular}{|c|c|c|c|}
\hline Phosphine $^{I}$ & $\mathbf{X}$ & $\mathbf{C}_{6} \mathbf{D}_{6}$ & Toluene-d 8 \\
\hline 3a $\left(\mathrm{PPh}_{2}\right)$ & $\mathrm{CO}_{2} t-\mathrm{Bu}$ & -14.7 & -14.8 \\
\hline 7a1 $\left(\mathrm{PPh}_{2}\right)$ & $\mathrm{CO}_{2} t-\mathrm{Bu}$ & -19.1 & -18.9 \\
\hline $7 \mathbf{a 2}\left(\mathrm{PPh}_{2}\right)$ & $\mathrm{CO}_{2} t-\mathrm{Bu}$ & $-18.7,-19.9$ & $-18.7,-19.8$ \\
\hline 3b(PPhi-Bu) & $\mathrm{CO}_{2} t-\mathrm{Bu}$ & -27.7 & -27.7 \\
\hline 7b1 (PPhi-Bu) & $\mathrm{CO}_{2} t-\mathrm{Bu}$ & $-32.0(\mathrm{a}),-32.5(\mathrm{~b})$ & $-32.0,-32.4$ \\
\hline 7bn (PPhi-Bu) & $\mathrm{CO}_{2} t-\mathrm{Bu}$ & $-31.7,-32.1,-32.4,-32.7$ & \\
\hline 3c (PMeIs) & $\mathrm{CO}_{2} t-\mathrm{Bu}$ & -47.1 & -47.2 \\
\hline 7c1 (PMeIs) & $\mathrm{CO}_{2} t-\mathrm{Bu}$ & $-51.5(\mathrm{a}),-52.4(\mathrm{~b})$ & $-51.3,-52.2$ \\
\hline 7cn (PMeIs) & $\mathrm{CO}_{2} t-\mathrm{Bu}$ & $-49.0,-51.2,-51.6$ & \\
\hline 3a' $\left(\mathrm{PPh}_{2}\right)^{\mathrm{a}}$ & $\mathrm{CN}$ & -15.3 & \\
\hline
\end{tabular}

${ }^{\text {a }}$ commercial product 
Crystallographic data for the complexes $\mathrm{Ph}_{2} \mathrm{PCH}_{2} \mathrm{CH}\left(\mathrm{CO}_{2} t-\mathrm{Bu}\right)(\mathrm{CH}(\mathrm{Ph})(\mathrm{OH}))(\mathbf{1 0 a}), \mathrm{Pt}((\mathrm{R}, \mathrm{R})-$ Me-Duphos $)(\mathrm{Me})\left(\mathrm{PPh}_{2}\right) \quad\left(\begin{array}{ll}\mathbf{1} & \mathbf{1}\end{array}\right), \quad \mathrm{Pt}(\mathrm{dppe})(\mathrm{Cl})\left(\mathrm{CH}(\mathrm{Me})\left(\mathrm{CO}_{2} t-\mathrm{Bu}\right)\right) \cdot \mathrm{CH}_{2} \mathrm{Cl}_{2} \quad\left(\mathbf{2 0} \cdot \mathbf{C H}_{2} \mathbf{C l}_{2}\right)$, $\mathrm{Pt}(\mathrm{dppe})(\mathrm{Me})\left(\mathrm{CH}(\mathrm{Me})\left(\mathrm{CO}_{2} t\right.\right.$-Bu $\left.)\right) \bullet \mathrm{THF}(\mathbf{2 1} \bullet \mathbf{T H F})$, and $\left[(\mathrm{Pt}(\mathrm{dppe})(\mathrm{Me}))_{2}\left(\mu-\mathrm{PPh}_{2}\right)\right][\mathrm{OH}] \cdot 2.5 \mathrm{C}_{7} \mathrm{H}_{8}$ $\left(17 \mathbf{a} \cdot 2.5 \mathbf{C}_{7} \mathbf{H}_{8}\right)$

Experimental for phosphine 10a. A colorless plate $0.35 \times 0.20 \times 0.10 \mathrm{~mm}$ in size was mounted on a Cryoloop with Paratone oil. Data were collected in a nitrogen gas stream at 100(2) K using phi and omega scans. Crystal-to-detector distance was $60 \mathrm{~mm}$ and exposure time was 5 seconds per frame using a scan width of $0.3^{\circ}$. Data collection was $99.9 \%$ complete to $25.00^{\circ}$ in $\theta$. A total of 10013 reflections were collected covering the indices, $-14<=h<=14,-12<=k<=12$, $14<=l<=14$. 5221 reflections were found to be symmetry independent, with an $\mathrm{R}_{\text {int }}$ of 0.0203 . Indexing and unit cell refinement indicated a primitive, monoclinic lattice. The space group was found to be P2(1) (No. 4). The data were integrated using the Bruker SAINT software program and scaled using the SADABS software program. Solution by direct methods (SIR-2004) produced a complete heavy-atom phasing model consistent with the proposed structure. All nonhydrogen atoms were refined anisotropically by full-matrix least-squares (SHELXL-97). All hydrogen atoms were placed using a riding model. Their positions were constrained relative to their parent atom using the appropriate HFIX command in SHELXL-97. 
Table 1. Crystal data and structure refinement for $\mathrm{Ph}_{2} \mathrm{PCH}_{2} \mathrm{CH}\left(\mathrm{CO}_{2} t-\mathrm{Bu}\right)(\mathrm{CHPh}(\mathrm{OH}))$, glu237.

$\begin{array}{ll}\text { X-ray ID } & \text { glu237 }\end{array}$

Sample/notebook ID

CS592

Empirical formula

C26 H29 O3 P

Formula weight

420.46

Temperature

$100(2) \mathrm{K}$

Wavelength

$0.71073 \AA$

Crystal system

Monoclinic

Space group

P2(1)

Unit cell dimensions

$\mathrm{a}=10.7290(11) \AA$

$\alpha=90^{\circ}$.

$\mathrm{b}=9.6080(10) \AA$

$\beta=92.169(2)^{\circ}$.

$\mathrm{c}=11.2870(11) \AA$

$\gamma=90^{\circ}$.

Volume

$1162.7(2) \AA^{3}$

Z

2

Density (calculated)

$1.201 \mathrm{Mg} / \mathrm{m}^{3}$

Absorption coefficient

$0.142 \mathrm{~mm}^{-1}$

$\mathrm{F}(000)$

448

Crystal size

$0.35 \times 0.20 \times 0.10 \mathrm{~mm}^{3}$

Crystal color/habit

colorless plate

Theta range for data collection

2.57 to $28.24^{\circ}$.

Index ranges

$-14<=\mathrm{h}<=14,-12<=\mathrm{k}<=12,-14<=1<=14$

Reflections collected

10013

Independent reflections

$5221[\mathrm{R}($ int $)=0.0203]$

Completeness to theta $=25.00^{\circ}$

$99.9 \%$

Absorption correction

Semi-empirical from equivalents

Max. and min. transmission

0.9860 and 0.9520

Refinement method

Full-matrix least-squares on $\mathrm{F}^{2}$

Data / restraints / parameters

$5221 / 1 / 275$

Goodness-of-fit on $\mathrm{F}^{2}$

1.045

Final $\mathrm{R}$ indices [I $>2 \operatorname{sigma}(\mathrm{I})]$

$\mathrm{R} 1=0.0414, w \mathrm{R} 2=0.1003$

$\mathrm{R}$ indices (all data)

$\mathrm{R} 1=0.0438, w \mathrm{R} 2=0.1019$ 
Absolute structure parameter

Largest diff. peak and hole
$-0.04(8)$

0.541 and -0.213 e. $\AA^{-3}$ 
Table 2. Atomic coordinates ( $\left.\times 10^{4}\right)$ and equivalent isotropic displacement parameters $\left(\AA^{2} \times 10^{3}\right)$

for $\mathrm{Ph}_{2} \mathrm{PCH}_{2} \mathrm{CH}\left(\mathrm{CO}_{2} t-\mathrm{Bu}\right)(\mathrm{CHPh}(\mathrm{OH}))$, glu237. U(eq) is defined as one third of the trace of the orthogonalized $\mathrm{U}^{\mathrm{ij}}$ tensor.

\begin{tabular}{|c|c|c|c|c|}
\hline & $\mathrm{x}$ & $\mathrm{y}$ & $\mathrm{z}$ & $\mathrm{U}(\mathrm{eq})$ \\
\hline$C(1)$ & $7043(2)$ & $6418(2)$ & $1417(2)$ & $21(1)$ \\
\hline$C(2)$ & $6735(2)$ & 7751(2) & 1793(2) & $28(1)$ \\
\hline$C(3)$ & $6087(2)$ & $8677(2)$ & 1042(2) & $36(1)$ \\
\hline$C(4)$ & $5746(2)$ & $8267(3)$ & $-96(2)$ & $35(1)$ \\
\hline$C(5)$ & 6064(2) & 6953(2) & $-494(2)$ & $32(1)$ \\
\hline$C(6)$ & 6711(2) & $6036(2)$ & $254(2)$ & $26(1)$ \\
\hline$C(7)$ & $8556(2)$ & $3944(2)$ & $1660(2)$ & 21(1) \\
\hline$C(8)$ & $7955(2)$ & $2777(2)$ & $1178(2)$ & $30(1)$ \\
\hline$C(9)$ & $8601(2)$ & $1813(2)$ & $516(2)$ & $35(1)$ \\
\hline$C(10)$ & $9849(2)$ & 2017(2) & $315(2)$ & $32(1)$ \\
\hline$C(11)$ & $10465(2)$ & $3151(2)$ & $797(2)$ & $31(1)$ \\
\hline$C(12)$ & $9827(2)$ & 4099(2) & $1478(2)$ & $24(1)$ \\
\hline$C(13)$ & $6462(2)$ & $4405(2)$ & $3118(2)$ & $22(1)$ \\
\hline$C(14)$ & $6857(2)$ & $3435(2)$ & $4138(2)$ & $19(1)$ \\
\hline$C(15)$ & $5756(2)$ & 2553(2) & $4559(2)$ & $22(1)$ \\
\hline$C(16)$ & $6165(2)$ & 1661(2) & $5599(2)$ & $29(1)$ \\
\hline$C(17)$ & $5950(2)$ & 2130(3) & $6750(2)$ & $34(1)$ \\
\hline$C(18)$ & $6347(2)$ & 1386(3) & $7736(2)$ & $45(1)$ \\
\hline$C(19)$ & 6966(2) & $177(4)$ & 7571(2) & $50(1)$ \\
\hline$C(20)$ & 7204(2) & $-326(3)$ & $6467(3)$ & $59(1)$ \\
\hline$C(21)$ & 6794(2) & $438(3)$ & $5450(2)$ & $44(1)$ \\
\hline $\mathrm{C}(22)$ & $7369(2)$ & $4304(2)$ & $5174(2)$ & 21(1) \\
\hline $\mathrm{C}(23)$ & 9197(2) & $4631(2)$ & $6512(2)$ & $27(1)$ \\
\hline$C(24)$ & $9459(3)$ & $6117(3)$ & $6169(2)$ & $41(1)$ \\
\hline$C(25)$ & 10404(3) & $3820(3)$ & 6599(3) & $64(1)$ \\
\hline
\end{tabular}


C(26)

$\mathrm{O}(1)$

$\mathrm{O}(2)$

$\mathrm{O}(3)$

$\mathrm{P}(1)$
8485(3)

4521(3)

1754(2)

5245(2)

3913(1)

5306(1)
7637(2)

3565(1)

5608(1)

5520(1)

2538(1)
53(1)

32(1)

33(1)

22(1)

19(1) 
Table 3. Bond lengths $[\AA]$ and angles $[\mathrm{deg}]$ for $\mathrm{Ph}_{2} \mathrm{PCH}_{2} \mathrm{CH}\left(\mathrm{CO}_{2} t-\mathrm{Bu}\right)(\mathrm{CHPh}(\mathrm{OH}))$, glu237.

\begin{tabular}{|c|c|c|c|}
\hline $\mathrm{C}(1)-\mathrm{C}(2)$ & $1.394(3)$ & $C(14)-C(15)$ & $1.544(2)$ \\
\hline $\mathrm{C}(1)-\mathrm{C}(6)$ & $1.396(3)$ & $\mathrm{C}(14)-\mathrm{H}(14)$ & 1.0000 \\
\hline $\mathrm{C}(1)-\mathrm{P}(1)$ & $1.8379(19)$ & $\mathrm{C}(15)-\mathrm{O}(1)$ & $1.423(2)$ \\
\hline $\mathrm{C}(2)-\mathrm{C}(3)$ & $1.395(3)$ & $C(15)-C(16)$ & $1.504(3)$ \\
\hline $\mathrm{C}(2)-\mathrm{H}(2)$ & 0.9500 & $\mathrm{C}(15)-\mathrm{H}(15)$ & 1.0000 \\
\hline $\mathrm{C}(3)-\mathrm{C}(4)$ & $1.379(4)$ & $C(16)-C(21)$ & $1.369(3)$ \\
\hline $\mathrm{C}(3)-\mathrm{H}(3)$ & 0.9500 & $C(16)-C(17)$ & $1.403(3)$ \\
\hline$C(4)-C(5)$ & $1.388(4)$ & $\mathrm{C}(17)-\mathrm{C}(18)$ & $1.377(3)$ \\
\hline $\mathrm{C}(4)-\mathrm{H}(4)$ & 0.9500 & $\mathrm{C}(17)-\mathrm{H}(17)$ & 0.9500 \\
\hline $\mathrm{C}(5)-\mathrm{C}(6)$ & $1.388(3)$ & $\mathrm{C}(18)-\mathrm{C}(19)$ & $1.355(4)$ \\
\hline $\mathrm{C}(5)-\mathrm{H}(5)$ & 0.9500 & $\mathrm{C}(18)-\mathrm{H}(18)$ & 0.9500 \\
\hline $\mathrm{C}(6)-\mathrm{H}(6)$ & 0.9500 & $C(19)-C(20)$ & $1.369(4)$ \\
\hline$C(7)-C(8)$ & $1.394(3)$ & $\mathrm{C}(19)-\mathrm{H}(19)$ & 0.9500 \\
\hline$C(7)-C(12)$ & $1.395(3)$ & $C(20)-C(21)$ & $1.419(4)$ \\
\hline $\mathrm{C}(7)-\mathrm{P}(1)$ & $1.832(2)$ & $\mathrm{C}(20)-\mathrm{H}(20)$ & 0.9500 \\
\hline $\mathrm{C}(8)-\mathrm{C}(9)$ & $1.391(3)$ & $\mathrm{C}(21)-\mathrm{H}(21)$ & 0.9500 \\
\hline $\mathrm{C}(8)-\mathrm{H}(8)$ & 0.9500 & $\mathrm{C}(22)-\mathrm{O}(2)$ & $1.214(2)$ \\
\hline$C(9)-C(10)$ & $1.380(3)$ & $\mathrm{C}(22)-\mathrm{O}(3)$ & $1.322(2)$ \\
\hline $\mathrm{C}(9)-\mathrm{H}(9)$ & 0.9500 & $\mathrm{C}(23)-\mathrm{O}(3)$ & $1.489(2)$ \\
\hline$C(10)-C(11)$ & $1.376(3)$ & $C(23)-C(24)$ & $1.508(3)$ \\
\hline $\mathrm{C}(10)-\mathrm{H}(10)$ & 0.9500 & $C(23)-C(26)$ & $1.511(3)$ \\
\hline$C(11)-C(12)$ & $1.388(3)$ & $C(23)-C(25)$ & $1.511(3)$ \\
\hline $\mathrm{C}(11)-\mathrm{H}(11)$ & 0.9500 & $\mathrm{C}(24)-\mathrm{H}(24 \mathrm{~A})$ & 0.9800 \\
\hline $\mathrm{C}(12)-\mathrm{H}(12)$ & 0.9500 & $\mathrm{C}(24)-\mathrm{H}(24 \mathrm{~B})$ & 0.9800 \\
\hline$C(13)-C(14)$ & $1.529(2)$ & $\mathrm{C}(24)-\mathrm{H}(24 \mathrm{C})$ & 0.9800 \\
\hline$C(13)-P(1)$ & $1.8468(18)$ & $\mathrm{C}(25)-\mathrm{H}(25 \mathrm{~A})$ & 0.9800 \\
\hline $\mathrm{C}(13)-\mathrm{H}(13 \mathrm{~A})$ & 0.9900 & $\mathrm{C}(25)-\mathrm{H}(25 \mathrm{~B})$ & 0.9800 \\
\hline $\mathrm{C}(13)-\mathrm{H}(13 \mathrm{~B})$ & 0.9900 & $\mathrm{C}(25)-\mathrm{H}(25 \mathrm{C})$ & 0.9800 \\
\hline$C(14)-C(22)$ & $1.522(3)$ & $\mathrm{C}(26)-\mathrm{H}(26 \mathrm{~A})$ & 0.9800 \\
\hline
\end{tabular}




\begin{tabular}{|c|c|c|c|}
\hline $\mathrm{C}(26)-\mathrm{H}(26 \mathrm{~B})$ & 0.9800 & $\mathrm{O}(1)-\mathrm{H}(1)$ & 0.8400 \\
\hline $\mathrm{C}(26)-\mathrm{H}(26 \mathrm{C})$ & 0.9800 & & \\
\hline$C(2)-C(1)-C(6)$ & $118.21(18)$ & $\mathrm{C}(11)-\mathrm{C}(10)-\mathrm{H}(10)$ & 119.9 \\
\hline $\mathrm{C}(2)-\mathrm{C}(1)-\mathrm{P}(1)$ & $115.66(15)$ & $\mathrm{C}(9)-\mathrm{C}(10)-\mathrm{H}(10)$ & 119.9 \\
\hline$C(6)-C(1)-P(1)$ & $126.11(15)$ & $C(10)-C(11)-C(12)$ & $119.89(19)$ \\
\hline$C(1)-C(2)-C(3)$ & $121.3(2)$ & $\mathrm{C}(10)-\mathrm{C}(11)-\mathrm{H}(11)$ & 120.1 \\
\hline $\mathrm{C}(1)-\mathrm{C}(2)-\mathrm{H}(2)$ & 119.3 & $\mathrm{C}(12)-\mathrm{C}(11)-\mathrm{H}(11)$ & 120.1 \\
\hline $\mathrm{C}(3)-\mathrm{C}(2)-\mathrm{H}(2)$ & 119.3 & $\mathrm{C}(11)-\mathrm{C}(12)-\mathrm{C}(7)$ & $121.13(19)$ \\
\hline$C(4)-C(3)-C(2)$ & $119.5(2)$ & $\mathrm{C}(11)-\mathrm{C}(12)-\mathrm{H}(12)$ & 119.4 \\
\hline $\mathrm{C}(4)-\mathrm{C}(3)-\mathrm{H}(3)$ & 120.3 & $\mathrm{C}(7)-\mathrm{C}(12)-\mathrm{H}(12)$ & 119.4 \\
\hline $\mathrm{C}(2)-\mathrm{C}(3)-\mathrm{H}(3)$ & 120.3 & $\mathrm{C}(14)-\mathrm{C}(13)-\mathrm{P}(1)$ & $110.53(12)$ \\
\hline$C(3)-C(4)-C(5)$ & $120.1(2)$ & $\mathrm{C}(14)-\mathrm{C}(13)-\mathrm{H}(13 \mathrm{~A})$ & 109.5 \\
\hline $\mathrm{C}(3)-\mathrm{C}(4)-\mathrm{H}(4)$ & 119.9 & $\mathrm{P}(1)-\mathrm{C}(13)-\mathrm{H}(13 \mathrm{~A})$ & 109.5 \\
\hline $\mathrm{C}(5)-\mathrm{C}(4)-\mathrm{H}(4)$ & 119.9 & $\mathrm{C}(14)-\mathrm{C}(13)-\mathrm{H}(13 \mathrm{~B})$ & 109.5 \\
\hline$C(4)-C(5)-C(6)$ & $120.2(2)$ & $\mathrm{P}(1)-\mathrm{C}(13)-\mathrm{H}(13 \mathrm{~B})$ & 109.5 \\
\hline $\mathrm{C}(4)-\mathrm{C}(5)-\mathrm{H}(5)$ & 119.9 & $\mathrm{H}(13 \mathrm{~A})-\mathrm{C}(13)-\mathrm{H}(13 \mathrm{~B})$ & 108.1 \\
\hline $\mathrm{C}(6)-\mathrm{C}(5)-\mathrm{H}(5)$ & 119.9 & $C(22)-C(14)-C(13)$ & $109.03(15)$ \\
\hline$C(5)-C(6)-C(1)$ & $120.6(2)$ & $\mathrm{C}(22)-\mathrm{C}(14)-\mathrm{C}(15)$ & $108.87(14)$ \\
\hline $\mathrm{C}(5)-\mathrm{C}(6)-\mathrm{H}(6)$ & 119.7 & $C(13)-C(14)-C(15)$ & $111.91(14)$ \\
\hline $\mathrm{C}(1)-\mathrm{C}(6)-\mathrm{H}(6)$ & 119.7 & $\mathrm{C}(22)-\mathrm{C}(14)-\mathrm{H}(14)$ & 109.0 \\
\hline $\mathrm{C}(8)-\mathrm{C}(7)-\mathrm{C}(12)$ & $117.94(18)$ & $\mathrm{C}(13)-\mathrm{C}(14)-\mathrm{H}(14)$ & 109.0 \\
\hline $\mathrm{C}(8)-\mathrm{C}(7)-\mathrm{P}(1)$ & $125.93(15)$ & $\mathrm{C}(15)-\mathrm{C}(14)-\mathrm{H}(14)$ & 109.0 \\
\hline $\mathrm{C}(12)-\mathrm{C}(7)-\mathrm{P}(1)$ & $116.13(14)$ & $\mathrm{O}(1)-\mathrm{C}(15)-\mathrm{C}(16)$ & $112.67(17)$ \\
\hline $\mathrm{C}(9)-\mathrm{C}(8)-\mathrm{C}(7)$ & $120.87(19)$ & $\mathrm{O}(1)-\mathrm{C}(15)-\mathrm{C}(14)$ & $106.60(14)$ \\
\hline $\mathrm{C}(9)-\mathrm{C}(8)-\mathrm{H}(8)$ & 119.6 & $\mathrm{C}(16)-\mathrm{C}(15)-\mathrm{C}(14)$ & $110.42(15)$ \\
\hline $\mathrm{C}(7)-\mathrm{C}(8)-\mathrm{H}(8)$ & 119.6 & $\mathrm{O}(1)-\mathrm{C}(15)-\mathrm{H}(15)$ & 109.0 \\
\hline $\mathrm{C}(10)-\mathrm{C}(9)-\mathrm{C}(8)$ & $120.0(2)$ & $\mathrm{C}(16)-\mathrm{C}(15)-\mathrm{H}(15)$ & 109.0 \\
\hline $\mathrm{C}(10)-\mathrm{C}(9)-\mathrm{H}(9)$ & 120.0 & $\mathrm{C}(14)-\mathrm{C}(15)-\mathrm{H}(15)$ & 109.0 \\
\hline $\mathrm{C}(8)-\mathrm{C}(9)-\mathrm{H}(9)$ & 120.0 & $\mathrm{C}(21)-\mathrm{C}(16)-\mathrm{C}(17)$ & $119.2(2)$ \\
\hline $\mathrm{C}(11)-\mathrm{C}(10)-\mathrm{C}(9)$ & $120.13(19)$ & $C(21)-C(16)-C(15)$ & $121.6(2)$ \\
\hline
\end{tabular}




\begin{tabular}{|c|c|c|c|}
\hline $\mathrm{C}(17)-\mathrm{C}(16)-\mathrm{C}(15)$ & $119.1(2)$ & $\mathrm{C}(23)-\mathrm{C}(25)-\mathrm{H}(25 \mathrm{~A})$ & 109.5 \\
\hline$C(18)-C(17)-C(16)$ & $121.7(2)$ & $\mathrm{C}(23)-\mathrm{C}(25)-\mathrm{H}(25 \mathrm{~B})$ & 109.5 \\
\hline $\mathrm{C}(18)-\mathrm{C}(17)-\mathrm{H}(17)$ & 119.2 & $\mathrm{H}(25 \mathrm{~A})-\mathrm{C}(25)-\mathrm{H}(25 \mathrm{~B})$ & 109.5 \\
\hline $\mathrm{C}(16)-\mathrm{C}(17)-\mathrm{H}(17)$ & 119.2 & $\mathrm{C}(23)-\mathrm{C}(25)-\mathrm{H}(25 \mathrm{C})$ & 109.5 \\
\hline $\mathrm{C}(19)-\mathrm{C}(18)-\mathrm{C}(17)$ & $118.2(3)$ & $\mathrm{H}(25 \mathrm{~A})-\mathrm{C}(25)-\mathrm{H}(25 \mathrm{C})$ & 109.5 \\
\hline $\mathrm{C}(19)-\mathrm{C}(18)-\mathrm{H}(18)$ & 120.9 & $\mathrm{H}(25 \mathrm{~B})-\mathrm{C}(25)-\mathrm{H}(25 \mathrm{C})$ & 109.5 \\
\hline $\mathrm{C}(17)-\mathrm{C}(18)-\mathrm{H}(18)$ & 120.9 & $\mathrm{C}(23)-\mathrm{C}(26)-\mathrm{H}(26 \mathrm{~A})$ & 109.5 \\
\hline$C(18)-C(19)-C(20)$ & $122.5(2)$ & $\mathrm{C}(23)-\mathrm{C}(26)-\mathrm{H}(26 \mathrm{~B})$ & 109.5 \\
\hline C(18)-C(19)-H(19) & 118.8 & $\mathrm{H}(26 \mathrm{~A})-\mathrm{C}(26)-\mathrm{H}(26 \mathrm{~B})$ & 109.5 \\
\hline C(20)-C(19)-H(19) & 118.8 & $\mathrm{C}(23)-\mathrm{C}(26)-\mathrm{H}(26 \mathrm{C})$ & 109.5 \\
\hline$C(19)-C(20)-C(21)$ & $119.4(3)$ & $\mathrm{H}(26 \mathrm{~A})-\mathrm{C}(26)-\mathrm{H}(26 \mathrm{C})$ & 109.5 \\
\hline $\mathrm{C}(19)-\mathrm{C}(20)-\mathrm{H}(20)$ & 120.3 & $\mathrm{H}(26 \mathrm{~B})-\mathrm{C}(26)-\mathrm{H}(26 \mathrm{C})$ & 109.5 \\
\hline $\mathrm{C}(21)-\mathrm{C}(20)-\mathrm{H}(20)$ & 120.3 & $\mathrm{C}(15)-\mathrm{O}(1)-\mathrm{H}(1)$ & 109.5 \\
\hline$C(16)-C(21)-C(20)$ & $119.0(3)$ & $\mathrm{C}(22)-\mathrm{O}(3)-\mathrm{C}(23)$ & $121.21(15)$ \\
\hline $\mathrm{C}(16)-\mathrm{C}(21)-\mathrm{H}(21)$ & 120.5 & $\mathrm{C}(7)-\mathrm{P}(1)-\mathrm{C}(1)$ & $103.65(8)$ \\
\hline $\mathrm{C}(20)-\mathrm{C}(21)-\mathrm{H}(21)$ & 120.5 & $C(7)-P(1)-C(13)$ & $102.47(9)$ \\
\hline $\mathrm{O}(2)-\mathrm{C}(22)-\mathrm{O}(3)$ & $125.23(17)$ & $\mathrm{C}(1)-\mathrm{P}(1)-\mathrm{C}(13)$ & $99.46(8)$ \\
\hline $\mathrm{O}(2)-\mathrm{C}(22)-\mathrm{C}(14)$ & $122.82(17)$ & & \\
\hline $\mathrm{O}(3)-\mathrm{C}(22)-\mathrm{C}(14)$ & $111.95(15)$ & $\begin{array}{l}\text { Symmetry transformations } \\
\text { equivalent atoms: }\end{array}$ & used to \\
\hline $\mathrm{O}(3)-\mathrm{C}(23)-\mathrm{C}(24)$ & $109.72(17)$ & & \\
\hline $\mathrm{O}(3)-\mathrm{C}(23)-\mathrm{C}(26)$ & $110.33(17)$ & & \\
\hline $\mathrm{C}(24)-\mathrm{C}(23)-\mathrm{C}(26)$ & $112.8(2)$ & & \\
\hline $\mathrm{O}(3)-\mathrm{C}(23)-\mathrm{C}(25)$ & $102.16(17)$ & & \\
\hline$C(24)-C(23)-C(25)$ & $109.7(2)$ & & \\
\hline$C(26)-C(23)-C(25)$ & $111.6(2)$ & & \\
\hline $\mathrm{C}(23)-\mathrm{C}(24)-\mathrm{H}(24 \mathrm{~A})$ & 109.5 & & \\
\hline $\mathrm{C}(23)-\mathrm{C}(24)-\mathrm{H}(24 \mathrm{~B})$ & 109.5 & & \\
\hline $\mathrm{H}(24 \mathrm{~A})-\mathrm{C}(24)-\mathrm{H}(24 \mathrm{~B})$ & 109.5 & & \\
\hline $\mathrm{C}(23)-\mathrm{C}(24)-\mathrm{H}(24 \mathrm{C})$ & 109.5 & & \\
\hline $\mathrm{H}(24 \mathrm{~A})-\mathrm{C}(24)-\mathrm{H}(24 \mathrm{C})$ & 109.5 & & \\
\hline $\mathrm{H}(24 \mathrm{~B})-\mathrm{C}(24)-\mathrm{H}(24 \mathrm{C})$ & 109.5 & & \\
\hline
\end{tabular}


Table 4. Anisotropic displacement parameters $\left(\AA^{2} \times 10^{3}\right)$ for $\mathrm{Ph}_{2} \mathrm{PCH}_{2} \mathrm{CH}\left(\mathrm{CO}_{2} t-\mathrm{Bu}\right)(\mathrm{CHPh}(\mathrm{OH}))$, glu237. The anisotropic displacement factor exponent takes the form: $-2 \pi^{2}\left[\mathrm{~h}^{2} \mathrm{a}^{* 2} \mathrm{U}^{11}+\ldots+2 \mathrm{~h} \mathrm{k} \mathrm{a} \mathrm{a}^{*} \mathrm{~b}^{*} \mathrm{U}^{12}\right]$

\begin{tabular}{|c|c|c|c|c|c|c|}
\hline & $\mathrm{U}^{11}$ & $\mathrm{U}^{22}$ & $\mathrm{U}^{33}$ & $\mathrm{U}^{23}$ & $\mathrm{U}^{13}$ & $\mathrm{U}^{12}$ \\
\hline$C(1)$ & $14(1)$ & $24(1)$ & $26(1)$ & $6(1)$ & $0(1)$ & $-2(1)$ \\
\hline$C(2)$ & $22(1)$ & $28(1)$ & $34(1)$ & 2(1) & $-2(1)$ & 1(1) \\
\hline$C(3)$ & $27(1)$ & $28(1)$ & $53(1)$ & $10(1)$ & $0(1)$ & $4(1)$ \\
\hline$C(4)$ & $22(1)$ & $38(1)$ & $44(1)$ & $18(1)$ & $-2(1)$ & 1(1) \\
\hline$C(5)$ & $24(1)$ & $45(1)$ & $27(1)$ & 11(1) & $-3(1)$ & $-5(1)$ \\
\hline$C(6)$ & $23(1)$ & $30(1)$ & $26(1)$ & $5(1)$ & $2(1)$ & $-3(1)$ \\
\hline$C(7)$ & $20(1)$ & $21(1)$ & $21(1)$ & $4(1)$ & $-1(1)$ & $0(1)$ \\
\hline $\mathrm{C}(8)$ & $21(1)$ & $30(1)$ & $38(1)$ & $-4(1)$ & $-3(1)$ & $-2(1)$ \\
\hline$C(9)$ & $34(1)$ & $32(1)$ & $40(1)$ & $-11(1)$ & $-6(1)$ & $0(1)$ \\
\hline$C(10)$ & $35(1)$ & $33(1)$ & $29(1)$ & $-5(1)$ & $0(1)$ & $10(1)$ \\
\hline $\mathrm{C}(11)$ & $22(1)$ & $34(1)$ & $38(1)$ & $0(1)$ & $4(1)$ & $5(1)$ \\
\hline$C(12)$ & $21(1)$ & $22(1)$ & $29(1)$ & $4(1)$ & $0(1)$ & 1(1) \\
\hline$C(13)$ & $16(1)$ & $27(1)$ & $22(1)$ & $4(1)$ & $0(1)$ & $1(1)$ \\
\hline$C(14)$ & $15(1)$ & $23(1)$ & $20(1)$ & 2(1) & $1(1)$ & $-1(1)$ \\
\hline$C(15)$ & $19(1)$ & $27(1)$ & $22(1)$ & 1(1) & $2(1)$ & $-3(1)$ \\
\hline$C(16)$ & $15(1)$ & $38(1)$ & $34(1)$ & $14(1)$ & $-1(1)$ & $-7(1)$ \\
\hline$C(17)$ & $28(1)$ & $48(1)$ & $27(1)$ & $10(1)$ & $-1(1)$ & $-15(1)$ \\
\hline C(18) & $31(1)$ & $68(2)$ & $35(1)$ & $17(1)$ & $-3(1)$ & $-16(1)$ \\
\hline$C(19)$ & $32(1)$ & $67(2)$ & $50(1)$ & $31(2)$ & $0(1)$ & $-9(1)$ \\
\hline$C(20)$ & $29(1)$ & $50(2)$ & $97(2)$ & $24(2)$ & $-1(1)$ & $9(1)$ \\
\hline $\mathrm{C}(21)$ & $29(1)$ & $40(1)$ & $62(2)$ & $14(1)$ & $9(1)$ & $0(1)$ \\
\hline$C(22)$ & $17(1)$ & $24(1)$ & $21(1)$ & 2(1) & $2(1)$ & $0(1)$ \\
\hline$C(23)$ & $25(1)$ & $26(1)$ & $30(1)$ & $-5(1)$ & $-8(1)$ & $-1(1)$ \\
\hline$C(24)$ & $49(1)$ & $29(1)$ & $44(1)$ & $-2(1)$ & $-9(1)$ & $-11(1)$ \\
\hline$C(25)$ & $45(2)$ & $56(2)$ & $89(2)$ & $-35(2)$ & $-41(2)$ & $22(1)$ \\
\hline$C(26)$ & $57(2)$ & $74(2)$ & $25(1)$ & $0(1)$ & $-8(1)$ & $-30(2)$ \\
\hline
\end{tabular}




\begin{tabular}{lllllll}
$\mathrm{O}(1)$ & $31(1)$ & $41(1)$ & $23(1)$ & $0(1)$ & $1(1)$ & $-17(1)$ \\
$\mathrm{O}(2)$ & $23(1)$ & $38(1)$ & $36(1)$ & $-12(1)$ & $-1(1)$ & $10(1)$ \\
$\mathrm{O}(3)$ & $20(1)$ & $21(1)$ & $25(1)$ & $-2(1)$ & $-5(1)$ & $2(1)$ \\
$\mathrm{P}(1)$ & $16(1)$ & $20(1)$ & $21(1)$ & $2(1)$ & $-1(1)$ & $-1(1)$ \\
\hline
\end{tabular}


Table 5. Hydrogen coordinates ( $\left.\times 10^{4}\right)$ and isotropic displacement parameters $\left(\AA^{2} \times 10^{3}\right)$ for $\mathrm{Ph}_{2} \mathrm{PCH}_{2} \mathrm{CH}\left(\mathrm{CO}_{2} t-\mathrm{Bu}\right)(\mathrm{CHPh}(\mathrm{OH}))$, glu237.

\begin{tabular}{|c|c|c|c|c|}
\hline & $\mathrm{X}$ & $\mathrm{y}$ & z & $\mathrm{U}(\mathrm{eq})$ \\
\hline $\mathrm{H}(2)$ & 6970 & 8036 & 2576 & 34 \\
\hline $\mathrm{H}(3)$ & 5883 & 9582 & 1313 & 43 \\
\hline $\mathrm{H}(4)$ & 5292 & 8886 & -607 & 42 \\
\hline $\mathrm{H}(5)$ & 5838 & 6680 & -1283 & 38 \\
\hline $\mathrm{H}(6)$ & 6929 & 5140 & -28 & 31 \\
\hline $\mathrm{H}(8)$ & 7093 & 2638 & 1303 & 36 \\
\hline $\mathrm{H}(9)$ & 8183 & 1015 & 202 & 42 \\
\hline $\mathrm{H}(10)$ & 10283 & 1373 & -156 & 39 \\
\hline $\mathrm{H}(11)$ & 11326 & 3286 & 664 & 38 \\
\hline $\mathrm{H}(12)$ & 10264 & 4865 & 1826 & 29 \\
\hline $\mathrm{H}(13 \mathrm{~A})$ & 5860 & 5101 & 3400 & 26 \\
\hline $\mathrm{H}(13 \mathrm{~B})$ & 6043 & 3859 & 2474 & 26 \\
\hline $\mathrm{H}(14)$ & 7527 & 2799 & 3869 & 23 \\
\hline $\mathrm{H}(15)$ & 5074 & 3188 & 4805 & 27 \\
\hline $\mathrm{H}(17)$ & 5518 & 2982 & 6852 & 41 \\
\hline $\mathrm{H}(18)$ & 6192 & 1712 & 8511 & 54 \\
\hline $\mathrm{H}(19)$ & 7245 & -343 & 8246 & 59 \\
\hline $\mathrm{H}(20)$ & 7641 & -1178 & 6383 & 71 \\
\hline $\mathrm{H}(21)$ & 6953 & 105 & 4677 & 52 \\
\hline $\mathrm{H}(24 \mathrm{~A})$ & 9865 & 6132 & 5406 & 62 \\
\hline $\mathrm{H}(24 \mathrm{~B})$ & 10008 & 6548 & 6777 & 62 \\
\hline $\mathrm{H}(24 \mathrm{C})$ & 8673 & 6635 & 6100 & 62 \\
\hline $\mathrm{H}(25 \mathrm{~A})$ & 10227 & 2848 & 6794 & 96 \\
\hline $\mathrm{H}(25 \mathrm{~B})$ & 10954 & 4224 & 7221 & 96 \\
\hline $\mathrm{H}(25 \mathrm{C})$ & 10814 & 3862 & 5838 & 96 \\
\hline
\end{tabular}




\begin{tabular}{llccc}
$\mathrm{H}(26 \mathrm{~A})$ & 7708 & 5054 & 7549 & 79 \\
$\mathrm{H}(26 \mathrm{~B})$ & 8995 & 4896 & 8302 & 79 \\
$\mathrm{H}(26 \mathrm{C})$ & 8291 & 3543 & 7791 & 79 \\
$\mathrm{H}(1)$ & 4736 & 1234 & 3767 & 47 \\
\hline
\end{tabular}


Table 1. Crystal data and structure refinement for $\mathrm{Pt}((\mathrm{R}, \mathrm{R})-\mathrm{Me}-\mathrm{Duphos})(\mathrm{Me})\left(\mathrm{PPh}_{2}\right)$, glu164.

Identification code

Empirical formula

Formula weight

Temperature

Wavelength

Crystal system

Space group

Unit cell dimensions

Volume

Z

Density (calculated)

Absorption coefficient

$\mathrm{F}(000)$

Crystal size

Theta range for data collection

Index ranges

Reflections collected

Independent reflections

Completeness to theta $=24.00^{\circ}$

Absorption correction

Max. and min. transmission

Refinement method

Data / restraints / parameters

Goodness-of-fit on $\mathrm{F}^{2}$

Final $\mathrm{R}$ indices $[\mathrm{I}>2 \operatorname{sigma}(\mathrm{I})]$

$\mathrm{R}$ indices (all data)

Absolute structure parameter

Largest diff. peak and hole glu 164

C31 H41 P3 Pt

701.64

213(2) K

$0.71073 \AA$

Monoclinic

P2(1)

$$
\begin{array}{ll}
\mathrm{a}=9.7369(14) \AA & \alpha=90^{\circ} \\
\mathrm{b}=19.017(3) \AA & \beta=91.064(3)^{\circ} \\
\mathrm{c}=15.778(2) \AA & \gamma=90^{\circ}
\end{array}
$$

2921.0(7) $\AA^{3}$

4

$1.595 \mathrm{~g} / \mathrm{cm}^{3}$

$4.986 \mathrm{~mm}^{-1}$

1400

$0.30 \times 0.15 \times 0.10 \mathrm{~mm}^{3}$

1.68 to $24.00^{\circ}$

$-10<=\mathrm{h}<=11,-21<=\mathrm{k}<=21,-17<=\mathrm{l}<=18$

15985

$9041[\mathrm{R}(\mathrm{int})=0.0290]$

$99.9 \%$

Semi-empirical from equivalents

1.000 and 0.686

Full-matrix least-squares on $\mathrm{F}^{2}$

$9041 / 1 / 631$

0.961

$\mathrm{R} 1=0.0287, \mathrm{wR} 2=0.0640$

$\mathrm{R} 1=0.0302, \mathrm{wR} 2=0.0646$

$0.012(5)$

1.602 and -0.823 e $\AA^{-3}$ 
Table 2. Atomic coordinates $\left(\mathrm{x} 10^{4}\right)$ and equivalent isotropic displacement parameters $\left(\AA^{2} \times 10^{3}\right)$ for $\operatorname{Pt}(\mathrm{R}, \mathrm{R})-\mathrm{Me}-$ Duphos) $(\mathrm{Me})\left(\mathrm{PPh}_{2}\right)$, glu164. $\mathrm{U}(\mathrm{eq})$ is defined as one third of the trace of the orthogonalized $\mathrm{U}^{\mathrm{ij}}$ tensor.

\begin{tabular}{|c|c|c|c|c|}
\hline & $\mathrm{x}$ & $\mathrm{y}$ & $\mathrm{z}$ & $\mathrm{U}(\mathrm{eq})$ \\
\hline $\mathrm{Pt}(1)$ & $5455(1)$ & $9502(1)$ & 9536(1) & $26(1)$ \\
\hline $\mathrm{P}(1)$ & $5585(2)$ & $8430(1)$ & 8931(1) & $27(1)$ \\
\hline $\mathrm{P}(2)$ & $3302(2)$ & 9554(1) & $8985(1)$ & $25(1)$ \\
\hline $\mathrm{P}(3)$ & $5176(2)$ & $10648(1)$ & 10119(1) & $29(1)$ \\
\hline $\mathrm{C}(1)$ & 7446(7) & 9416(4) & $10062(5)$ & $51(2)$ \\
\hline$C(2)$ & 4027(7) & $8257(4)$ & $8307(3)$ & $25(1)$ \\
\hline$C(3)$ & $3817(7)$ & 7653(4) & 7831(4) & $33(2)$ \\
\hline C(4) & $2585(8)$ & 7541(4) & 7394(4) & $43(2)$ \\
\hline$C(5)$ & $1555(8)$ & $8036(4)$ & 7448(4) & $39(2)$ \\
\hline$C(6)$ & $1720(7)$ & $8630(4)$ & 7926(4) & $34(2)$ \\
\hline$C(7)$ & 2946(7) & 8761(3) & $8367(3)$ & $27(1)$ \\
\hline$C(8)$ & $7109(7)$ & $8247(4)$ & $8295(4)$ & $40(2)$ \\
\hline $\mathrm{C}(9)$ & $7725(8)$ & $7558(4)$ & $8642(5)$ & $55(2)$ \\
\hline $\mathrm{C}(10)$ & $7348(8)$ & 7489(4) & 9552(5) & $50(2)$ \\
\hline $\mathrm{C}(11)$ & $5804(7)$ & 7644(4) & 9603(4) & $34(2)$ \\
\hline$C(12)$ & 6917(9) & $8263(5)$ & $7315(5)$ & $64(2)$ \\
\hline$C(13)$ & $5242(8)$ & 7712(4) & 10493(4) & $44(2)$ \\
\hline$C(14)$ & 2666(7) & 10279(3) & $8278(4)$ & $31(2)$ \\
\hline$C(15)$ & 1667(7) & $10678(4)$ & 8833(4) & $41(2)$ \\
\hline$C(16)$ & $862(7)$ & 10157(4) & 9340(4) & $39(2)$ \\
\hline $\mathrm{C}(17)$ & $1900(6)$ & $9647(4)$ & 9759(4) & $32(2)$ \\
\hline$C(18)$ & $3787(8)$ & 10722(4) & 7875(4) & $41(2)$ \\
\hline $\mathrm{C}(19)$ & 1301(7) & 8973(4) & 10094(4) & $43(2)$ \\
\hline$C(20)$ & $6772(6)$ & 11135(3) & 9945(4) & $26(1)$ \\
\hline $\mathrm{C}(21)$ & 7297(7) & 11153(3) & 9134(4) & $34(2)$ \\
\hline $\mathrm{C}(22)$ & $8352(7)$ & $11595(4)$ & $8928(4)$ & $39(2)$ \\
\hline
\end{tabular}




\begin{tabular}{|c|c|c|c|c|}
\hline $\mathrm{C}(23)$ & $8934(7)$ & $12029(4)$ & 9519(4) & $40(2)$ \\
\hline$C(24)$ & $8440(7)$ & $12014(4)$ & $10335(4)$ & $39(2)$ \\
\hline$C(25)$ & 7390(7) & $11575(3)$ & $10542(4)$ & $34(2)$ \\
\hline$C(26)$ & $5135(7)$ & 10553(4) & $11285(4)$ & $35(2)$ \\
\hline $\mathrm{C}(27)$ & $6276(8)$ & 10368(4) & 11792(4) & $49(2)$ \\
\hline$C(28)$ & $6159(9)$ & $10331(5)$ & $12660(5)$ & $59(2)$ \\
\hline$C(29)$ & $4924(10)$ & 10467(6) & $13037(5)$ & $72(3)$ \\
\hline$C(30)$ & $3794(9)$ & $10648(6)$ & $12529(5)$ & $67(3)$ \\
\hline $\mathrm{C}(31)$ & $3907(8)$ & 10695(4) & $11659(5)$ & $48(2)$ \\
\hline $\operatorname{Pt}(2)$ & $9529(1)$ & 6392(1) & $4412(1)$ & $29(1)$ \\
\hline $\mathrm{P}(4)$ & $9509(2)$ & $7458(1)$ & $3782(1)$ & $28(1)$ \\
\hline $\mathrm{P}(5)$ & $11721(2)$ & $6298(1)$ & $3984(1)$ & $27(1)$ \\
\hline $\mathrm{P}(6)$ & $9735(2)$ & $5296(1)$ & $5123(1)$ & $30(1)$ \\
\hline$C(32)$ & 7496(7) & $6496(4)$ & 4794(5) & $54(2)$ \\
\hline$C(33)$ & $11136(7)$ & 7595(4) & $3255(4)$ & $30(2)$ \\
\hline$C(34)$ & $11444(7)$ & $8193(4)$ & $2776(4)$ & $36(2)$ \\
\hline $\mathrm{C}(35)$ & $12712(8)$ & $8276(4)$ & $2422(4)$ & $45(2)$ \\
\hline$C(36)$ & $13710(7)$ & 7772(4) & $2550(4)$ & $41(2)$ \\
\hline$C(37)$ & $13457(7)$ & 7182(4) & $3017(4)$ & $36(2)$ \\
\hline$C(38)$ & $12165(7)$ & 7078(3) & $3381(4)$ & $28(2)$ \\
\hline C(39) & $8067(7)$ & $7675(4)$ & $3046(4)$ & $37(2)$ \\
\hline $\mathrm{C}(40)$ & 7618(8) & $8420(4)$ & $3276(5)$ & $50(2)$ \\
\hline $\mathrm{C}(41)$ & 7836(8) & $8510(4)$ & $4219(5)$ & $48(2)$ \\
\hline $\mathrm{C}(42)$ & $9307(7)$ & $8253(4)$ & $4443(4)$ & $35(2)$ \\
\hline $\mathrm{C}(43)$ & $8335(8)$ & $7570(4)$ & 2097(4) & $51(2)$ \\
\hline $\mathrm{C}(44)$ & $9590(8)$ & $8162(5)$ & $5375(4)$ & $53(2)$ \\
\hline $\mathrm{C}(45)$ & $12295(6)$ & $5560(3)$ & $3306(4)$ & $30(2)$ \\
\hline $\mathrm{C}(46)$ & 13281(7) & $5128(4)$ & $3888(4)$ & $39(2)$ \\
\hline $\mathrm{C}(47)$ & $14106(7)$ & $5654(4)$ & $4422(4)$ & $40(2)$ \\
\hline $\mathrm{C}(48)$ & 13087(7) & $6157(4)$ & $4813(4)$ & $34(2)$ \\
\hline $\mathrm{C}(49)$ & $11178(7)$ & $5115(4)$ & $2903(4)$ & $44(2)$ \\
\hline
\end{tabular}




\begin{tabular}{lrrrr}
$\mathrm{C}(50)$ & $13687(8)$ & $6832(4)$ & $5183(4)$ & $48(2)$ \\
$\mathrm{C}(51)$ & $8133(7)$ & $4797(3)$ & $4986(4)$ & $30(2)$ \\
$\mathrm{C}(52)$ & $7706(7)$ & $4642(4)$ & $4159(4)$ & $43(2)$ \\
$\mathrm{C}(53)$ & $6637(8)$ & $4198(4)$ & $3976(4)$ & $46(2)$ \\
$\mathrm{C}(54)$ & $5921(8)$ & $3895(4)$ & $4616(5)$ & $47(2)$ \\
$\mathrm{C}(55)$ & $6284(9)$ & $4049(5)$ & $5440(5)$ & $59(2)$ \\
$\mathrm{C}(56)$ & $7380(7)$ & $4501(4)$ & $5617(4)$ & $44(2)$ \\
$\mathrm{C}(57)$ & $9757(7)$ & $5513(3)$ & $6267(4)$ & $31(2)$ \\
$\mathrm{C}(58)$ & $9718(8)$ & $6200(4)$ & $6573(5)$ & $46(2)$ \\
$\mathrm{C}(59)$ & $9845(8)$ & $6319(5)$ & $7455(5)$ & $61(2)$ \\
$\mathrm{C}(60)$ & $9978(8)$ & $5779(5)$ & $8017(5)$ & $53(2)$ \\
$\mathrm{C}(61)$ & $10043(9)$ & $5105(5)$ & $7717(5)$ & $59(2)$ \\
$\mathrm{C}(62)$ & $9950(8)$ & $4980(4)$ & $6858(4)$ & $48(2)$ \\
\hline
\end{tabular}


Table 3. Bond lengths $[\AA ̊]$ and angles [deg] for $\mathrm{Pt}((\mathrm{R}, \mathrm{R})-\mathrm{Me}-\mathrm{Duphos})(\mathrm{Me})\left(\mathrm{PPh}_{2}\right)$, glu164.

\begin{tabular}{|c|c|c|c|}
\hline $\operatorname{Pt}(1)-\mathrm{C}(1)$ & $2.101(6)$ & $C(20)-C(25)$ & $1.389(8)$ \\
\hline $\mathrm{Pt}(1)-\mathrm{P}(1)$ & $2.2555(17)$ & $\mathrm{C}(21)-\mathrm{C}(22)$ & $1.371(9)$ \\
\hline $\mathrm{Pt}(1)-\mathrm{P}(2)$ & $2.2567(14)$ & $\mathrm{C}(22)-\mathrm{C}(23)$ & 1.361(9) \\
\hline $\mathrm{Pt}(1)-\mathrm{P}(3)$ & $2.3822(18)$ & $\mathrm{C}(23)-\mathrm{C}(24)$ & $1.384(10)$ \\
\hline$P(1)-C(2)$ & $1.822(6)$ & $C(24)-C(25)$ & $1.365(10)$ \\
\hline $\mathrm{P}(1)-\mathrm{C}(8)$ & $1.840(7)$ & $\mathrm{C}(26)-\mathrm{C}(31)$ & $1.371(10)$ \\
\hline$P(1)-C(11)$ & $1.843(7)$ & $C(26)-C(27)$ & $1.403(9)$ \\
\hline$P(2)-C(7)$ & $1.825(6)$ & $\mathrm{C}(27)-\mathrm{C}(28)$ & $1.378(10)$ \\
\hline$P(2)-C(17)$ & $1.857(7)$ & $C(28)-C(29)$ & $1.376(12)$ \\
\hline$P(2)-C(14)$ & $1.872(6)$ & $\mathrm{C}(29)-\mathrm{C}(30)$ & $1.391(12)$ \\
\hline$P(3)-C(20)$ & $1.834(7)$ & $\mathrm{C}(30)-\mathrm{C}(31)$ & $1.383(10)$ \\
\hline$P(3)-C(26)$ & $1.849(7)$ & $\operatorname{Pt}(2)-C(32)$ & $2.089(7)$ \\
\hline $\mathrm{C}(2)-\mathrm{C}(3)$ & $1.386(9)$ & $\mathrm{Pt}(2)-\mathrm{P}(5)$ & $2.2573(17)$ \\
\hline$C(2)-C(7)$ & $1.427(9)$ & $\mathrm{Pt}(2)-\mathrm{P}(4)$ & $2.2573(18)$ \\
\hline $\mathrm{C}(3)-\mathrm{C}(4)$ & $1.389(9)$ & $\mathrm{Pt}(2)-\mathrm{P}(6)$ & $2.3735(18)$ \\
\hline $\mathrm{C}(4)-\mathrm{C}(5)$ & $1.380(10)$ & $\mathrm{P}(4)-\mathrm{C}(33)$ & $1.821(7)$ \\
\hline$C(5)-C(6)$ & $1.365(9)$ & $\mathrm{P}(4)-\mathrm{C}(42)$ & $1.849(7)$ \\
\hline $\mathrm{C}(6)-\mathrm{C}(7)$ & $1.393(8)$ & $\mathrm{P}(4)-\mathrm{C}(39)$ & $1.852(6)$ \\
\hline $\mathrm{C}(8)-\mathrm{C}(9)$ & $1.536(10)$ & $\mathrm{P}(5)-\mathrm{C}(38)$ & $1.818(7)$ \\
\hline $\mathrm{C}(8)-\mathrm{C}(12)$ & $1.555(10)$ & $\mathrm{P}(5)-\mathrm{C}(45)$ & $1.858(6)$ \\
\hline $\mathrm{C}(9)-\mathrm{C}(10)$ & $1.494(11)$ & $\mathrm{P}(5)-\mathrm{C}(48)$ & $1.867(5)$ \\
\hline $\mathrm{C}(10)-\mathrm{C}(11)$ & $1.535(10)$ & $\mathrm{P}(6)-\mathrm{C}(51)$ & $1.835(7)$ \\
\hline $\mathrm{C}(11)-\mathrm{C}(13)$ & $1.523(9)$ & $\mathrm{P}(6)-\mathrm{C}(57)$ & $1.851(6)$ \\
\hline $\mathrm{C}(14)-\mathrm{C}(15)$ & $1.525(10)$ & $\mathrm{C}(33)-\mathrm{C}(34)$ & 1.401(9) \\
\hline $\mathrm{C}(14)-\mathrm{C}(18)$ & $1.527(10)$ & $\mathrm{C}(33)-\mathrm{C}(38)$ & 1.416(9) \\
\hline$C(15)-C(16)$ & $1.503(10)$ & $\mathrm{C}(34)-\mathrm{C}(35)$ & $1.374(10)$ \\
\hline$C(16)-C(17)$ & $1.541(9)$ & $C(35)-C(36)$ & $1.376(10)$ \\
\hline $\mathrm{C}(17)-\mathrm{C}(19)$ & $1.508(9)$ & $\mathrm{C}(36)-\mathrm{C}(37)$ & $1.367(10)$ \\
\hline $\mathrm{C}(20)-\mathrm{C}(21)$ & $1.387(9)$ & $\mathrm{C}(37)-\mathrm{C}(38)$ & $1.406(9)$ \\
\hline
\end{tabular}




\begin{tabular}{|c|c|c|c|}
\hline C(39)-C(40) & $1.528(10)$ & $\mathrm{C}(8)-\mathrm{P}(1)-\mathrm{C}(11)$ & $94.2(3)$ \\
\hline C(39)-C(43) & $1.537(9)$ & $\mathrm{C}(2)-\mathrm{P}(1)-\mathrm{Pt}(1)$ & $109.8(2)$ \\
\hline$C(40)-C(41)$ & $1.509(10)$ & $\mathrm{C}(8)-\mathrm{P}(1)-\mathrm{Pt}(1)$ & $117.0(3)$ \\
\hline $\mathrm{C}(41)-\mathrm{C}(42)$ & $1.547(10)$ & $\mathrm{C}(11)-\mathrm{P}(1)-\mathrm{Pt}(1)$ & $119.8(2)$ \\
\hline$C(42)-C(44)$ & $1.501(8)$ & $\mathrm{C}(7)-\mathrm{P}(2)-\mathrm{C}(17)$ & 107.2(3) \\
\hline $\mathrm{C}(45)-\mathrm{C}(49)$ & $1.509(9)$ & $\mathrm{C}(7)-\mathrm{P}(2)-\mathrm{C}(14)$ & $103.5(3)$ \\
\hline $\mathrm{C}(45)-\mathrm{C}(46)$ & $1.552(8)$ & $\mathrm{C}(17)-\mathrm{P}(2)-\mathrm{C}(14)$ & $94.7(3)$ \\
\hline C(46)-C(47) & $1.526(9)$ & $\mathrm{C}(7)-\mathrm{P}(2)-\mathrm{Pt}(1)$ & $109.6(2)$ \\
\hline $\mathrm{C}(47)-\mathrm{C}(48)$ & $1.519(10)$ & $\mathrm{C}(17)-\mathrm{P}(2)-\mathrm{Pt}(1)$ & $116.10(17)$ \\
\hline C(48)-C(50) & $1.522(10)$ & $\mathrm{C}(14)-\mathrm{P}(2)-\mathrm{Pt}(1)$ & $123.8(2)$ \\
\hline$C(51)-C(56)$ & $1.368(9)$ & $\mathrm{C}(20)-\mathrm{P}(3)-\mathrm{C}(26)$ & $103.4(3)$ \\
\hline$C(51)-C(52)$ & $1.394(8)$ & $\mathrm{C}(20)-\mathrm{P}(3)-\mathrm{Pt}(1)$ & $107.5(2)$ \\
\hline $\mathrm{C}(52)-\mathrm{C}(53)$ & $1.367(10)$ & $\mathrm{C}(26)-\mathrm{P}(3)-\mathrm{Pt}(1)$ & $107.5(2)$ \\
\hline$C(53)-C(54)$ & $1.365(10)$ & $\mathrm{C}(3)-\mathrm{C}(2)-\mathrm{C}(7)$ & $119.4(6)$ \\
\hline $\mathrm{C}(54)-\mathrm{C}(55)$ & $1.372(10)$ & $\mathrm{C}(3)-\mathrm{C}(2)-\mathrm{P}(1)$ & $123.7(5)$ \\
\hline$C(55)-C(56)$ & $1.394(10)$ & $\mathrm{C}(7)-\mathrm{C}(2)-\mathrm{P}(1)$ & $116.8(5)$ \\
\hline$C(57)-C(62)$ & $1.388(10)$ & $\mathrm{C}(2)-\mathrm{C}(3)-\mathrm{C}(4)$ & $120.9(7)$ \\
\hline $\mathrm{C}(57)-\mathrm{C}(58)$ & $1.393(10)$ & $\mathrm{C}(5)-\mathrm{C}(4)-\mathrm{C}(3)$ & 119.1(7) \\
\hline $\mathrm{C}(58)-\mathrm{C}(59)$ & $1.412(10)$ & $\mathrm{C}(6)-\mathrm{C}(5)-\mathrm{C}(4)$ & 121.3(6) \\
\hline$C(59)-C(60)$ & $1.361(12)$ & $C(5)-C(6)-C(7)$ & $121.0(7)$ \\
\hline$C(60)-C(61)$ & $1.369(12)$ & $C(6)-C(7)-C(2)$ & $118.2(6)$ \\
\hline \multirow[t]{2}{*}{$C(61)-C(62)$} & $1.376(10)$ & $\mathrm{C}(6)-\mathrm{C}(7)-\mathrm{P}(2)$ & $124.5(5)$ \\
\hline & & $\mathrm{C}(2)-\mathrm{C}(7)-\mathrm{P}(2)$ & $117.2(4)$ \\
\hline$C(1)-\operatorname{Pt}(1)-P(1)$ & $92.2(2)$ & $\mathrm{C}(9)-\mathrm{C}(8)-\mathrm{C}(12)$ & 114.2(7) \\
\hline $\mathrm{C}(1)-\operatorname{Pt}(1)-\mathrm{P}(2)$ & $177.9(2)$ & $\mathrm{C}(9)-\mathrm{C}(8)-\mathrm{P}(1)$ & $106.3(5)$ \\
\hline $\mathrm{P}(1)-\mathrm{Pt}(1)-\mathrm{P}(2)$ & $86.27(6)$ & $\mathrm{C}(12)-\mathrm{C}(8)-\mathrm{P}(1)$ & $117.1(5)$ \\
\hline $\mathrm{C}(1)-\operatorname{Pt}(1)-\mathrm{P}(3)$ & $91.7(2)$ & $\mathrm{C}(10)-\mathrm{C}(9)-\mathrm{C}(8)$ & $108.4(6)$ \\
\hline $\mathrm{P}(1)-\mathrm{Pt}(1)-\mathrm{P}(3)$ & $176.03(6)$ & $C(9)-C(10)-C(11)$ & $106.9(6)$ \\
\hline $\mathrm{P}(2)-\mathrm{Pt}(1)-\mathrm{P}(3)$ & $89.84(6)$ & $\mathrm{C}(13)-\mathrm{C}(11)-\mathrm{C}(10)$ & $115.7(6)$ \\
\hline $\mathrm{C}(2)-\mathrm{P}(1)-\mathrm{C}(8)$ & 110.1(3) & $\mathrm{C}(13)-\mathrm{C}(11)-\mathrm{P}(1)$ & $114.9(5)$ \\
\hline $\mathrm{C}(2)-\mathrm{P}(1)-\mathrm{C}(11)$ & $104.5(3)$ & $\mathrm{C}(10)-\mathrm{C}(11)-\mathrm{P}(1)$ & $103.3(5)$ \\
\hline
\end{tabular}




\begin{tabular}{|c|c|c|c|}
\hline $\mathrm{C}(15)-\mathrm{C}(14)-\mathrm{C}(18)$ & $115.7(6)$ & $\mathrm{C}(33)-\mathrm{P}(4)-\mathrm{C}(39)$ & 109.7(3) \\
\hline $\mathrm{C}(15)-\mathrm{C}(14)-\mathrm{P}(2)$ & $103.4(4)$ & $\mathrm{C}(42)-\mathrm{P}(4)-\mathrm{C}(39)$ & $94.8(3)$ \\
\hline $\mathrm{C}(18)-\mathrm{C}(14)-\mathrm{P}(2)$ & $115.1(5)$ & $\mathrm{C}(33)-\mathrm{P}(4)-\mathrm{Pt}(2)$ & $109.2(2)$ \\
\hline $\mathrm{C}(16)-\mathrm{C}(15)-\mathrm{C}(14)$ & $108.8(6)$ & $\mathrm{C}(42)-\mathrm{P}(4)-\mathrm{Pt}(2)$ & 119.1(2) \\
\hline$C(15)-C(16)-C(17)$ & $107.4(6)$ & $\mathrm{C}(39)-\mathrm{P}(4)-\mathrm{Pt}(2)$ & $118.5(3)$ \\
\hline$C(19)-C(17)-C(16)$ & $115.5(5)$ & $\mathrm{C}(38)-\mathrm{P}(5)-\mathrm{C}(45)$ & 103.7(3) \\
\hline C(19)-C(17)-P(2) & $116.3(5)$ & $\mathrm{C}(38)-\mathrm{P}(5)-\mathrm{C}(48)$ & 108.1(3) \\
\hline $\mathrm{C}(16)-\mathrm{C}(17)-\mathrm{P}(2)$ & $105.2(4)$ & $\mathrm{C}(45)-\mathrm{P}(5)-\mathrm{C}(48)$ & $94.4(3)$ \\
\hline $\mathrm{C}(21)-\mathrm{C}(20)-\mathrm{C}(25)$ & $116.6(6)$ & $\mathrm{C}(38)-\mathrm{P}(5)-\mathrm{Pt}(2)$ & 109.1(2) \\
\hline $\mathrm{C}(21)-\mathrm{C}(20)-\mathrm{P}(3)$ & $118.6(4)$ & $\mathrm{C}(45)-\mathrm{P}(5)-\mathrm{Pt}(2)$ & $121.9(2)$ \\
\hline $\mathrm{C}(25)-\mathrm{C}(20)-\mathrm{P}(3)$ & $124.1(5)$ & $\mathrm{C}(48)-\mathrm{P}(5)-\mathrm{Pt}(2)$ & $117.8(2)$ \\
\hline $\mathrm{C}(22)-\mathrm{C}(21)-\mathrm{C}(20)$ & 121.7(6) & $\mathrm{C}(51)-\mathrm{P}(6)-\mathrm{C}(57)$ & $103.0(3)$ \\
\hline $\mathrm{C}(23)-\mathrm{C}(22)-\mathrm{C}(21)$ & $120.9(7)$ & $\mathrm{C}(51)-\mathrm{P}(6)-\mathrm{Pt}(2)$ & $109.5(2)$ \\
\hline $\mathrm{C}(22)-\mathrm{C}(23)-\mathrm{C}(24)$ & $118.5(7)$ & $\mathrm{C}(57)-\mathrm{P}(6)-\mathrm{Pt}(2)$ & $105.3(2)$ \\
\hline $\mathrm{C}(25)-\mathrm{C}(24)-\mathrm{C}(23)$ & $120.7(6)$ & $\mathrm{C}(34)-\mathrm{C}(33)-\mathrm{C}(38)$ & $118.8(6)$ \\
\hline $\mathrm{C}(24)-\mathrm{C}(25)-\mathrm{C}(20)$ & $121.6(6)$ & $\mathrm{C}(34)-\mathrm{C}(33)-\mathrm{P}(4)$ & $124.0(5)$ \\
\hline$C(31)-C(26)-C(27)$ & $119.5(7)$ & $\mathrm{C}(38)-\mathrm{C}(33)-\mathrm{P}(4)$ & $117.1(5)$ \\
\hline $\mathrm{C}(31)-\mathrm{C}(26)-\mathrm{P}(3)$ & $116.4(5)$ & $\mathrm{C}(35)-\mathrm{C}(34)-\mathrm{C}(33)$ & $121.0(7)$ \\
\hline $\mathrm{C}(27)-\mathrm{C}(26)-\mathrm{P}(3)$ & $124.1(6)$ & $\mathrm{C}(34)-\mathrm{C}(35)-\mathrm{C}(36)$ & $119.8(7)$ \\
\hline $\mathrm{C}(28)-\mathrm{C}(27)-\mathrm{C}(26)$ & $120.1(8)$ & $\mathrm{C}(37)-\mathrm{C}(36)-\mathrm{C}(35)$ & 121.1(7) \\
\hline $\mathrm{C}(29)-\mathrm{C}(28)-\mathrm{C}(27)$ & $120.6(7)$ & $\mathrm{C}(36)-\mathrm{C}(37)-\mathrm{C}(38)$ & $120.5(7)$ \\
\hline $\mathrm{C}(28)-\mathrm{C}(29)-\mathrm{C}(30)$ & $119.1(7)$ & $\mathrm{C}(37)-\mathrm{C}(38)-\mathrm{C}(33)$ & $118.7(6)$ \\
\hline $\mathrm{C}(31)-\mathrm{C}(30)-\mathrm{C}(29)$ & $120.8(8)$ & $\mathrm{C}(37)-\mathrm{C}(38)-\mathrm{P}(5)$ & $123.5(5)$ \\
\hline $\mathrm{C}(26)-\mathrm{C}(31)-\mathrm{C}(30)$ & $120.0(7)$ & $\mathrm{C}(33)-\mathrm{C}(38)-\mathrm{P}(5)$ & $117.7(5)$ \\
\hline $\mathrm{C}(32)-\mathrm{Pt}(2)-\mathrm{P}(5)$ & $178.9(2)$ & $\mathrm{C}(40)-\mathrm{C}(39)-\mathrm{C}(43)$ & $114.0(6)$ \\
\hline $\mathrm{C}(32)-\mathrm{Pt}(2)-\mathrm{P}(4)$ & $92.4(2)$ & C(40)-C(39)-P(4) & $105.9(5)$ \\
\hline $\mathrm{P}(5)-\mathrm{Pt}(2)-\mathrm{P}(4)$ & $86.55(6)$ & $\mathrm{C}(43)-\mathrm{C}(39)-\mathrm{P}(4)$ & $116.2(5)$ \\
\hline $\mathrm{C}(32)-\mathrm{Pt}(2)-\mathrm{P}(6)$ & 91.1(2) & $\mathrm{C}(41)-\mathrm{C}(40)-\mathrm{C}(39)$ & $107.7(6)$ \\
\hline $\mathrm{P}(5)-\mathrm{Pt}(2)-\mathrm{P}(6)$ & $90.01(6)$ & $\mathrm{C}(40)-\mathrm{C}(41)-\mathrm{C}(42)$ & $107.6(6)$ \\
\hline $\mathrm{P}(4)-\mathrm{Pt}(2)-\mathrm{P}(6)$ & $175.19(6)$ & $C(44)-C(42)-C(41)$ & $114.3(6)$ \\
\hline $\mathrm{C}(33)-\mathrm{P}(4)-\mathrm{C}(42)$ & $104.0(3)$ & C(44)-C(42)-P(4) & $116.0(5)$ \\
\hline
\end{tabular}




$\begin{array}{llll}\mathrm{C}(41)-\mathrm{C}(42)-\mathrm{P}(4) & 103.7(4) & \mathrm{C}(54)-\mathrm{C}(53)-\mathrm{C}(52) & 120.1(6) \\ \mathrm{C}(49)-\mathrm{C}(45)-\mathrm{C}(46) & 112.7(6) & \mathrm{C}(53)-\mathrm{C}(54)-\mathrm{C}(55) & 119.0(7) \\ \mathrm{C}(49)-\mathrm{C}(45)-\mathrm{P}(5) & 116.4(5) & \mathrm{C}(54)-\mathrm{C}(55)-\mathrm{C}(56) & 120.2(7) \\ \mathrm{C}(46)-\mathrm{C}(45)-\mathrm{P}(5) & 104.4(4) & \mathrm{C}(51)-\mathrm{C}(56)-\mathrm{C}(55) & 121.8(6) \\ \mathrm{C}(47)-\mathrm{C}(46)-\mathrm{C}(45) & 107.1(5) & \mathrm{C}(62)-\mathrm{C}(57)-\mathrm{C}(58) & 117.1(6) \\ \mathrm{C}(48)-\mathrm{C}(47)-\mathrm{C}(46) & 107.2(6) & \mathrm{C}(62)-\mathrm{C}(57)-\mathrm{P}(6) & 119.5(5) \\ \mathrm{C}(47)-\mathrm{C}(48)-\mathrm{C}(50) & 116.0(6) & \mathrm{C}(58)-\mathrm{C}(57)-\mathrm{P}(6) & 123.2(5) \\ \mathrm{C}(47)-\mathrm{C}(48)-\mathrm{P}(5) & 105.5(4) & \mathrm{C}(57)-\mathrm{C}(58)-\mathrm{C}(59) & 119.3(7) \\ \mathrm{C}(50)-\mathrm{C}(48)-\mathrm{P}(5) & 114.3(5) & \mathrm{C}(60)-\mathrm{C}(59)-\mathrm{C}(58) & 121.8(8) \\ \mathrm{C}(56)-\mathrm{C}(51)-\mathrm{C}(52) & 116.1(6) & \mathrm{C}(59)-\mathrm{C}(60)-\mathrm{C}(61) & 119.0(7) \\ \mathrm{C}(56)-\mathrm{C}(51)-\mathrm{P}(6) & 126.4(5) & \mathrm{C}(60)-\mathrm{C}(61)-\mathrm{C}(62) & 120.0(8) \\ \mathrm{C}(52)-\mathrm{C}(51)-\mathrm{P}(6) & 117.2(5) & \mathrm{C}(61)-\mathrm{C}(62)-\mathrm{C}(57) & 122.8(8) \\ \mathrm{C}(53)-\mathrm{C}(52)-\mathrm{C}(51) & 122.7(7) & & \end{array}$

Symmetry transformations used to generate equivalent atoms: 
Table 4. Anisotropic displacement parameters $\left(\AA^{2} x \quad 10^{3}\right)$ for $\left.\mathrm{Pt}(\mathrm{R}, \mathrm{R})-\mathrm{Me}-\mathrm{Duphos}\right)(\mathrm{Me})\left(\mathrm{PPh}_{2}\right)$, glu164. The anisotropic displacement factor exponent takes the form: $-2 \pi^{2}\left[h^{2} a^{* 2} U^{11}+\ldots+2 h k a^{*} b^{*} U^{12}\right]$

\begin{tabular}{|c|c|c|c|c|c|c|}
\hline & $\mathrm{U}^{11}$ & $\mathrm{U}^{22}$ & $\mathrm{U}^{33}$ & $\mathrm{U}^{23}$ & $\mathrm{U}^{13}$ & $\mathrm{U}^{12}$ \\
\hline $\operatorname{Pt}(1)$ & $24(1)$ & $24(1)$ & $30(1)$ & $-2(1)$ & $-1(1)$ & $0(1)$ \\
\hline $\mathrm{P}(1)$ & $27(1)$ & $23(1)$ & $30(1)$ & $0(1)$ & $2(1)$ & $3(1)$ \\
\hline $\mathrm{P}(2)$ & $26(1)$ & $25(1)$ & $23(1)$ & $1(1)$ & $-1(1)$ & $1(1)$ \\
\hline $\mathrm{P}(3)$ & $29(1)$ & $29(1)$ & $28(1)$ & $-3(1)$ & 1(1) & $2(1)$ \\
\hline $\mathrm{C}(1)$ & $26(4)$ & $39(4)$ & $85(5)$ & $-17(4)$ & $-19(4)$ & $7(4)$ \\
\hline $\mathrm{C}(2)$ & $33(4)$ & $28(4)$ & $13(3)$ & $2(3)$ & $4(2)$ & $3(3)$ \\
\hline$C(3)$ & $46(4)$ & $25(4)$ & $28(3)$ & $1(3)$ & $3(3)$ & $-3(3)$ \\
\hline $\mathrm{C}(4)$ & $59(5)$ & $43(4)$ & $26(3)$ & $-1(3)$ & $2(3)$ & $-11(4)$ \\
\hline $\mathrm{C}(5)$ & $48(5)$ & $44(5)$ & $26(3)$ & $3(3)$ & $-11(3)$ & $-7(4)$ \\
\hline$C(6)$ & $32(4)$ & $39(4)$ & $30(3)$ & $3(3)$ & $-4(3)$ & $2(3)$ \\
\hline$C(7)$ & $34(4)$ & $27(4)$ & $20(3)$ & $8(3)$ & $3(3)$ & $-2(3)$ \\
\hline$C(8)$ & $29(4)$ & $42(4)$ & $50(4)$ & $1(4)$ & $9(3)$ & $5(3)$ \\
\hline $\mathrm{C}(9)$ & $40(5)$ & $43(5)$ & $82(6)$ & $-11(5)$ & $12(4)$ & $16(4)$ \\
\hline$C(10)$ & $42(5)$ & $44(5)$ & $64(5)$ & $11(4)$ & $-3(4)$ & $13(4)$ \\
\hline $\mathrm{C}(11)$ & $41(4)$ & $26(4)$ & $35(4)$ & $0(3)$ & $0(3)$ & $-4(3)$ \\
\hline$C(12)$ & $59(6)$ & $69(6)$ & $66(5)$ & $13(5)$ & $27(4)$ & $5(5)$ \\
\hline$C(13)$ & $58(5)$ & $41(5)$ & $31(4)$ & $9(3)$ & $-5(3)$ & $-7(4)$ \\
\hline$C(14)$ & $34(4)$ & $23(3)$ & $35(4)$ & $4(3)$ & $-6(3)$ & $3(3)$ \\
\hline$C(15)$ & $40(4)$ & $35(4)$ & $48(4)$ & $0(3)$ & $-6(3)$ & $7(3)$ \\
\hline$C(16)$ & $29(4)$ & $35(4)$ & $52(4)$ & $-5(3)$ & $7(3)$ & $6(3)$ \\
\hline$C(17)$ & $29(4)$ & $39(4)$ & $26(3)$ & $-6(3)$ & $0(2)$ & $3(3)$ \\
\hline$C(18)$ & $54(5)$ & $38(4)$ & $32(4)$ & $10(3)$ & $3(3)$ & $7(4)$ \\
\hline$C(19)$ & $43(4)$ & $47(5)$ & $39(4)$ & $12(3)$ & $0(3)$ & $5(4)$ \\
\hline$C(20)$ & $27(4)$ & $23(3)$ & $28(3)$ & $0(3)$ & $-3(3)$ & 2(3) \\
\hline$C(21)$ & $39(4)$ & $32(4)$ & $31(3)$ & $-5(3)$ & $-7(3)$ & 1(3) \\
\hline$C(22)$ & $31(4)$ & $52(5)$ & $36(4)$ & 13(3) & $4(3)$ & $-2(3)$ \\
\hline
\end{tabular}




\begin{tabular}{|c|c|c|c|c|c|c|}
\hline$C(23)$ & $33(4)$ & $38(4)$ & $50(5)$ & $9(4)$ & $3(3)$ & $-10(3)$ \\
\hline$C(24)$ & $38(4)$ & 41(4) & $37(4)$ & $-11(3)$ & $-7(3)$ & $-3(3)$ \\
\hline$C(25)$ & $40(4)$ & $37(4)$ & $23(3)$ & $-8(3)$ & $3(3)$ & 1(3) \\
\hline$C(26)$ & $34(4)$ & $32(4)$ & $39(4)$ & $0(3)$ & $-3(3)$ & $-2(3)$ \\
\hline$C(27)$ & $47(5)$ & $63(6)$ & $37(4)$ & 2(4) & $5(3)$ & $8(4)$ \\
\hline$C(28)$ & $51(6)$ & $78(7)$ & $48(5)$ & $14(4)$ & $-11(4)$ & $-4(5)$ \\
\hline C(29) & $67(7)$ & 117(9) & $31(4)$ & $0(5)$ & $7(4)$ & $-14(6)$ \\
\hline $\mathrm{C}(30)$ & $51(6)$ & 108(9) & $43(5)$ & $-5(5)$ & $17(4)$ & $3(5)$ \\
\hline $\mathrm{C}(31)$ & $35(4)$ & $59(5)$ & $51(5)$ & 1(4) & $-4(3)$ & $0(4)$ \\
\hline $\operatorname{Pt}(2)$ & $26(1)$ & $25(1)$ & $36(1)$ & 2(1) & $5(1)$ & $0(1)$ \\
\hline $\mathrm{P}(4)$ & $28(1)$ & $24(1)$ & $32(1)$ & $-2(1)$ & 1(1) & $3(1)$ \\
\hline $\mathrm{P}(5)$ & $26(1)$ & $30(1)$ & $26(1)$ & $2(1)$ & 2(1) & $3(1)$ \\
\hline $\mathrm{P}(6)$ & $31(1)$ & $25(1)$ & $34(1)$ & $1(1)$ & $4(1)$ & $0(1)$ \\
\hline$C(32)$ & $31(4)$ & $37(5)$ & $94(6)$ & $17(5)$ & 24(4) & $3(4)$ \\
\hline $\mathrm{C}(33)$ & $37(4)$ & 21(3) & $30(3)$ & $-5(3)$ & $-4(3)$ & $-8(3)$ \\
\hline$C(34)$ & $48(5)$ & $31(4)$ & 29(3) & 2(3) & $6(3)$ & $-7(3)$ \\
\hline$C(35)$ & $66(6)$ & $34(4)$ & $35(4)$ & $3(3)$ & $9(3)$ & $-8(4)$ \\
\hline$C(36)$ & $38(4)$ & $45(5)$ & $41(4)$ & $-5(3)$ & $15(3)$ & $-13(4)$ \\
\hline $\mathrm{C}(37)$ & $35(4)$ & $38(4)$ & $35(4)$ & $-3(3)$ & $6(3)$ & $0(3)$ \\
\hline C(38) & $28(4)$ & $31(4)$ & $25(3)$ & $-8(3)$ & $-5(3)$ & $-3(3)$ \\
\hline C(39) & $36(4)$ & $35(4)$ & 41(4) & $-3(3)$ & $-3(3)$ & $6(3)$ \\
\hline $\mathrm{C}(40)$ & $46(5)$ & $39(4)$ & $66(5)$ & $-2(4)$ & $-20(4)$ & $12(4)$ \\
\hline $\mathrm{C}(41)$ & $42(5)$ & $40(5)$ & $62(5)$ & $-19(4)$ & $5(4)$ & $7(4)$ \\
\hline $\mathrm{C}(42)$ & $37(4)$ & $30(4)$ & $38(4)$ & $-2(3)$ & $2(3)$ & $3(3)$ \\
\hline $\mathrm{C}(43)$ & $54(5)$ & $53(5)$ & $45(4)$ & $-8(4)$ & $-17(4)$ & $9(4)$ \\
\hline $\mathrm{C}(44)$ & $77(6)$ & $42(5)$ & $39(4)$ & $-1(3)$ & $6(4)$ & $-2(4)$ \\
\hline $\mathrm{C}(45)$ & $29(4)$ & $34(4)$ & $27(3)$ & $-1(3)$ & $6(3)$ & 7(3) \\
\hline $\mathrm{C}(46)$ & $32(4)$ & $35(4)$ & $51(4)$ & $-7(3)$ & $-7(3)$ & $8(3)$ \\
\hline C(47) & $38(4)$ & $48(5)$ & $33(4)$ & $-5(3)$ & $-5(3)$ & $15(4)$ \\
\hline C(48) & $29(4)$ & 44(4) & $28(3)$ & $0(3)$ & $-6(3)$ & $-1(3)$ \\
\hline $\mathrm{C}(49)$ & $44(5)$ & $44(5)$ & $45(4)$ & $-12(4)$ & $-8(3)$ & $7(4)$ \\
\hline
\end{tabular}




\begin{tabular}{lllllll}
$\mathrm{C}(50)$ & $50(5)$ & $65(5)$ & $29(4)$ & $-14(3)$ & $-7(3)$ & $6(4)$ \\
$\mathrm{C}(51)$ & $32(4)$ & $25(3)$ & $33(3)$ & $2(3)$ & $0(3)$ & $5(3)$ \\
$\mathrm{C}(52)$ & $44(4)$ & $56(5)$ & $31(3)$ & $2(3)$ & $9(3)$ & $-11(4)$ \\
$\mathrm{C}(53)$ & $41(5)$ & $62(5)$ & $35(4)$ & $-13(4)$ & $-2(3)$ & $7(4)$ \\
$\mathrm{C}(54)$ & $48(5)$ & $34(4)$ & $57(5)$ & $10(4)$ & $-11(4)$ & $-7(4)$ \\
$\mathrm{C}(55)$ & $53(6)$ & $75(6)$ & $49(5)$ & $22(4)$ & $-7(4)$ & $-27(5)$ \\
$\mathrm{C}(56)$ & $52(4)$ & $52(4)$ & $27(3)$ & $8(4)$ & $0(3)$ & $-8(4)$ \\
$\mathrm{C}(57)$ & $32(4)$ & $25(4)$ & $35(4)$ & $-7(3)$ & $7(3)$ & $-5(3)$ \\
$\mathrm{C}(58)$ & $41(5)$ & $46(5)$ & $51(4)$ & $-1(4)$ & $16(3)$ & $-9(4)$ \\
$\mathrm{C}(59)$ & $72(6)$ & $58(6)$ & $55(5)$ & $-26(5)$ & $27(4)$ & $-24(5)$ \\
$\mathrm{C}(60)$ & $58(5)$ & $70(6)$ & $32(4)$ & $-8(4)$ & $6(3)$ & $-20(5)$ \\
$\mathrm{C}(61)$ & $72(6)$ & $59(6)$ & $46(5)$ & $5(4)$ & $-12(4)$ & $-12(5)$ \\
$\mathrm{C}(62)$ & $63(5)$ & $44(5)$ & $36(4)$ & $-10(3)$ & $-13(4)$ & $3(4)$ \\
& & & & & & \\
\hline
\end{tabular}


Table 5. Hydrogen coordinates $\left(\mathrm{x} 10^{4}\right)$ and isotropic displacement parameters $\left(\AA^{2} \times 10^{3}\right)$ for $\mathrm{Pt}((\mathrm{R}, \mathrm{R})-\mathrm{Me}-$ Duphos)(Me)( $\left(\mathrm{PPh}_{2}\right)$, glu164.

\begin{tabular}{|c|c|c|c|c|}
\hline & $\mathrm{x}$ & $\mathrm{y}$ & $\mathrm{z}$ & $\mathrm{U}(\mathrm{eq})$ \\
\hline $\mathrm{H}(1 \mathrm{~A})$ & 7834 & 8964 & 9913 & 76 \\
\hline $\mathrm{H}(1 \mathrm{~B})$ & 7406 & 9455 & 10674 & 76 \\
\hline $\mathrm{H}(1 \mathrm{C})$ & 8018 & 9790 & 9843 & 76 \\
\hline $\mathrm{H}(3 \mathrm{~A})$ & 4517 & 7314 & 7805 & 40 \\
\hline $\mathrm{H}(4 \mathrm{~A})$ & 2456 & 7133 & 7065 & 51 \\
\hline $\mathrm{H}(5 \mathrm{~A})$ & 723 & 7964 & 7149 & 47 \\
\hline $\mathrm{H}(6 \mathrm{~A})$ & 996 & 8955 & 7958 & 41 \\
\hline $\mathrm{H}(8 \mathrm{~A})$ & 7783 & 8620 & 8438 & 48 \\
\hline $\mathrm{H}(9 \mathrm{~A})$ & 7365 & 7158 & 8317 & 66 \\
\hline $\mathrm{H}(9 \mathrm{~B})$ & 8727 & 7565 & 8592 & 66 \\
\hline $\mathrm{H}(10 \mathrm{~A})$ & 7873 & 7823 & 9901 & 60 \\
\hline $\mathrm{H}(10 \mathrm{~B})$ & 7546 & 7012 & 9756 & 60 \\
\hline $\mathrm{H}(11 \mathrm{~A})$ & 5310 & 7252 & 9319 & 40 \\
\hline $\mathrm{H}(12 \mathrm{~A})$ & 7787 & 8161 & 7051 & 97 \\
\hline $\mathrm{H}(12 \mathrm{~B})$ & 6601 & 8725 & 7140 & 97 \\
\hline $\mathrm{H}(12 \mathrm{C})$ & 6244 & 7912 & 7143 & 97 \\
\hline $\mathrm{H}(13 \mathrm{~A})$ & 5402 & 7277 & 10802 & 65 \\
\hline $\mathrm{H}(13 \mathrm{~B})$ & 4264 & 7806 & 10459 & 65 \\
\hline $\mathrm{H}(13 \mathrm{C})$ & 5703 & 8096 & 10787 & 65 \\
\hline $\mathrm{H}(14 \mathrm{~A})$ & 2123 & 10060 & 7812 & 37 \\
\hline $\mathrm{H}(15 \mathrm{~A})$ & 2174 & 10995 & 9216 & 49 \\
\hline $\mathrm{H}(15 \mathrm{~B})$ & 1042 & 10961 & 8479 & 49 \\
\hline $\mathrm{H}(16 \mathrm{~A})$ & 222 & 9898 & 8968 & 46 \\
\hline $\mathrm{H}(16 \mathrm{~B})$ & 334 & 10400 & 9774 & 46 \\
\hline $\mathrm{H}(17 \mathrm{~A})$ & 2301 & 9898 & 10254 & 38 \\
\hline $\mathrm{H}(18 \mathrm{~A})$ & 3367 & 11081 & 7518 & 62 \\
\hline
\end{tabular}




\begin{tabular}{|c|c|c|c|c|}
\hline $\mathrm{H}(18 \mathrm{~B})$ & 4364 & 10423 & 7533 & 62 \\
\hline $\mathrm{H}(18 \mathrm{C})$ & 4341 & 10944 & 8316 & 62 \\
\hline $\mathrm{H}(19 \mathrm{~A})$ & 588 & 9082 & 10494 & 65 \\
\hline $\mathrm{H}(19 \mathrm{~B})$ & 2018 & 8701 & 10376 & 65 \\
\hline $\mathrm{H}(19 \mathrm{C})$ & 911 & 8702 & 9627 & 65 \\
\hline $\mathrm{H}(21 \mathrm{~A})$ & 6921 & 10855 & 8716 & 41 \\
\hline $\mathrm{H}(22 \mathrm{~A})$ & 8678 & 11598 & 8371 & 47 \\
\hline $\mathrm{H}(23 \mathrm{~A})$ & 9655 & 12333 & 9375 & 48 \\
\hline $\mathrm{H}(24 \mathrm{~A})$ & 8832 & 12310 & 10752 & 47 \\
\hline $\mathrm{H}(25 \mathrm{~A})$ & 7077 & 11570 & 11102 & 40 \\
\hline $\mathrm{H}(27 \mathrm{~A})$ & 7122 & 10269 & 11540 & 59 \\
\hline $\mathrm{H}(28 \mathrm{~A})$ & 6929 & 10212 & 12998 & 71 \\
\hline $\mathrm{H}(29 \mathrm{~A})$ & 4844 & 10439 & 13629 & 86 \\
\hline $\mathrm{H}(30 \mathrm{~A})$ & 2945 & 10738 & 12781 & 81 \\
\hline $\mathrm{H}(31 \mathrm{~A})$ & 3140 & 10825 & 11323 & 58 \\
\hline $\mathrm{H}(32 \mathrm{~A})$ & 7117 & 6932 & 4573 & 81 \\
\hline $\mathrm{H}(32 \mathrm{~B})$ & 7469 & 6501 & 5408 & 81 \\
\hline $\mathrm{H}(32 \mathrm{C})$ & 6959 & 6103 & 4578 & 81 \\
\hline $\mathrm{H}(34 \mathrm{~A})$ & 10772 & 8543 & 2695 & 43 \\
\hline $\mathrm{H}(35 \mathrm{~A})$ & 12899 & 8676 & 2094 & 54 \\
\hline $\mathrm{H}(36 \mathrm{~A})$ & 14579 & 7834 & 2311 & 49 \\
\hline $\mathrm{H}(37 \mathrm{~A})$ & 14152 & 6844 & 3096 & 43 \\
\hline $\mathrm{H}(39 \mathrm{~A})$ & 7298 & 7359 & 3190 & 45 \\
\hline $\mathrm{H}(40 \mathrm{~A})$ & 8163 & 8766 & 2969 & 61 \\
\hline $\mathrm{H}(40 \mathrm{~B})$ & 6647 & 8489 & 3124 & 61 \\
\hline $\mathrm{H}(41 \mathrm{~A})$ & 7159 & 8233 & 4528 & 57 \\
\hline $\mathrm{H}(41 \mathrm{~B})$ & 7732 & 9005 & 4377 & 57 \\
\hline $\mathrm{H}(42 \mathrm{~A})$ & 9955 & 8611 & 4234 & 42 \\
\hline $\mathrm{H}(43 \mathrm{~A})$ & 7516 & 7692 & 1771 & 76 \\
\hline $\mathrm{H}(43 \mathrm{~B})$ & 8571 & 7083 & 1994 & 76 \\
\hline $\mathrm{H}(43 \mathrm{C})$ & 9089 & 7870 & 1929 & 76 \\
\hline
\end{tabular}




\begin{tabular}{|c|c|c|c|c|}
\hline $\mathrm{H}(44 \mathrm{~A})$ & 9462 & 8608 & 5662 & 79 \\
\hline $\mathrm{H}(44 \mathrm{~B})$ & 10528 & 8003 & 5464 & 79 \\
\hline $\mathrm{H}(44 \mathrm{C})$ & 8963 & 7816 & 5601 & 79 \\
\hline $\mathrm{H}(45 \mathrm{~A})$ & 12846 & 5762 & 2844 & 36 \\
\hline $\mathrm{H}(46 \mathrm{~A})$ & 12757 & 4815 & 4254 & 47 \\
\hline $\mathrm{H}(46 \mathrm{~B})$ & 13898 & 4841 & 3546 & 47 \\
\hline $\mathrm{H}(47 \mathrm{~A})$ & 14632 & 5408 & 4867 & 48 \\
\hline $\mathrm{H}(47 \mathrm{~B})$ & 14749 & 5911 & 4066 & 48 \\
\hline $\mathrm{H}(48 \mathrm{~A})$ & 12653 & 5903 & 5286 & 40 \\
\hline $\mathrm{H}(49 \mathrm{~A})$ & 11590 & 4748 & 2563 & 66 \\
\hline $\mathrm{H}(49 \mathrm{~B})$ & 10595 & 5408 & 2543 & 66 \\
\hline $\mathrm{H}(49 \mathrm{C})$ & 10631 & 4903 & 3342 & 66 \\
\hline $\mathrm{H}(50 \mathrm{~A})$ & 14375 & 6717 & 5613 & 72 \\
\hline $\mathrm{H}(50 \mathrm{~B})$ & 12961 & 7107 & 5435 & 72 \\
\hline $\mathrm{H}(50 \mathrm{C})$ & 14108 & 7103 & 4736 & 72 \\
\hline $\mathrm{H}(52 \mathrm{~A})$ & 8173 & 4851 & 3708 & 52 \\
\hline $\mathrm{H}(53 \mathrm{~A})$ & 6395 & 4100 & 3409 & 55 \\
\hline $\mathrm{H}(54 \mathrm{~A})$ & 5191 & 3586 & 4495 & 56 \\
\hline $\mathrm{H}(55 \mathrm{~A})$ & 5793 & 3848 & 5887 & 71 \\
\hline $\mathrm{H}(56 \mathrm{~A})$ & 7607 & 4605 & 6185 & 52 \\
\hline $\mathrm{H}(58 \mathrm{~A})$ & 9607 & 6580 & 6197 & 55 \\
\hline $\mathrm{H}(59 \mathrm{~A})$ & 9838 & 6783 & 7659 & 73 \\
\hline $\mathrm{H}(60 \mathrm{~A})$ & 10025 & 5868 & 8603 & 64 \\
\hline $\mathrm{H}(61 \mathrm{~A})$ & 10151 & 4727 & 8097 & 71 \\
\hline $\mathrm{H}(62 \mathrm{~A})$ & 10019 & 4514 & 6665 & 57 \\
\hline
\end{tabular}


Table 1. Crystal data and structure refinement for $\mathrm{Pt}(\mathrm{dppe})\left(\mathrm{CH}(\mathrm{Me}) \mathrm{CO}_{2} \mathrm{t}-\mathrm{Bu}\right)(\mathrm{Cl}) \cdot \mathrm{CH}_{2} \mathrm{Cl}_{2}$, glu167a.

Identification code

Empirical formula

Formula weight

Temperature

Wavelength

Crystal system

Space group

Unit cell dimensions

Volume

Z

Density (calculated)

Absorption coefficient

$\mathrm{F}(000)$

Crystal size

Theta range for data collection

Index ranges

Reflections collected

Independent reflections

Completeness to theta $=28.25^{\circ}$

Absorption correction

Max. and min. transmission

Refinement method

Data / restraints / parameters

Goodness-of-fit on $\mathrm{F}^{2}$

Final $\mathrm{R}$ indices [I $>2 \operatorname{sigma}(\mathrm{I})]$

$\mathrm{R}$ indices (all data)

Absolute structure parameter

Largest diff. peak and hole glu167a

$\mathrm{C}_{34} \mathrm{H}_{39} \mathrm{Cl}_{3} \mathrm{O}_{2} \mathrm{P}_{2} \mathrm{Pt}$

843.03

213(2) K

$0.71073 \AA$

Orthorhombic

Pna2(1)

$\mathrm{a}=17.3082(10) \AA$

$\alpha=90^{\circ}$.

$\mathrm{b}=10.2818(6) \AA$

$\beta=90^{\circ}$.

$c=19.0494(11) \AA$

$\gamma=90^{\circ}$.
4

$1.652 \mathrm{Mg} / \mathrm{m}^{3}$

$4.500 \mathrm{~mm}^{-1}$

1672

$0.15 \times 0.08 \times 0.06 \mathrm{~mm}^{3}$

2.14 to $28.25^{\circ}$.

$-22 \leq \mathrm{h} \leq 22,-13 \leq \mathrm{k} \leq 13,-25 \leq 1 \leq 23$

24635

$7635[\mathrm{R}(\mathrm{int})=0.0364]$

$97.2 \%$

Semi-empirical from equivalents

1.000 and 0.690

Full-matrix least-squares on $\mathrm{F}^{2}$

7635 / 3 / 379

1.007

$\mathrm{R} 1=0.0422, \mathrm{wR} 2=0.1037$

$\mathrm{R} 1=0.0494, \mathrm{wR} 2=0.1075$

$0.047(9)$

2.025 and -0.885 e. $\AA^{-3}$ 
Table 2. Atomic coordinates $\left(\mathrm{x} 10^{4}\right)$ and equivalent isotropic displacement parameters $\left(\AA^{2} \mathrm{x} 10^{3}\right)$ for $\mathrm{Pt}(\mathrm{dppe})\left(\mathrm{CH}(\mathrm{Me}) \mathrm{CO}_{2} \mathrm{t}-\mathrm{Bu}\right)(\mathrm{Cl}) \cdot \mathrm{CH}_{2} \mathrm{Cl}_{2}$,glu167a. U(eq) is defined as one third of the trace of the orthogonalized $\mathrm{U}^{\mathrm{ij}}$ tensor.

\begin{tabular}{|c|c|c|c|c|}
\hline & $\mathrm{x}$ & $\mathrm{y}$ & $\mathrm{z}$ & $\mathrm{U}(\mathrm{eq})$ \\
\hline $\operatorname{Pt}(1)$ & $6487(1)$ & 1187(1) & $5370(1)$ & $31(1)$ \\
\hline $\mathrm{Cl}(1)$ & $6814(2)$ & $225(2)$ & $4284(1)$ & $59(1)$ \\
\hline $\mathrm{P}(1)$ & 6092(1) & $2080(2)$ & $6362(1)$ & $32(1)$ \\
\hline $\mathrm{P}(2)$ & $6747(1)$ & $3262(2)$ & $5008(1)$ & $33(1)$ \\
\hline $\mathrm{O}(1)$ & 7653(3) & $-980(5)$ & $5898(3)$ & $43(1)$ \\
\hline $\mathrm{O}(2)$ & 7015(3) & $-2506(4)$ & $5258(4)$ & $50(2)$ \\
\hline$C(1)$ & 6306(4) & $-718(7)$ & $5772(5)$ & $40(2)$ \\
\hline$C(2)$ & 6997(5) & $-1513(8)$ & $5599(4)$ & $44(2)$ \\
\hline$C(3)$ & $5576(4)$ & $-1375(7)$ & $5478(10)$ & $62(4)$ \\
\hline$C(4)$ & $8397(5)$ & $-1607(11)$ & $5821(6)$ & $46(2)$ \\
\hline$C(5)$ & $8426(6)$ & $-2926(10)$ & $6115(6)$ & $71(3)$ \\
\hline$C(6)$ & $8924(6)$ & $-655(11)$ & $6252(7)$ & $72(3)$ \\
\hline$C(7)$ & $8637(6)$ & $-1562(13)$ & $5072(7)$ & $62(3)$ \\
\hline $\mathrm{C}(8)$ & $5884(5)$ & 3792(6) & 6172(4) & $38(2)$ \\
\hline $\mathrm{C}(9)$ & $6589(4)$ & $4317(6)$ & $5764(4)$ & $35(2)$ \\
\hline$C(10)$ & $5218(5)$ & 1453(8) & 6751(4) & $42(2)$ \\
\hline$C(11)$ & $4538(6)$ & $1556(12)$ & $6407(7)$ & $69(3)$ \\
\hline$C(12)$ & $3869(6)$ & $1075(12)$ & $6717(9)$ & $85(4)$ \\
\hline$C(13)$ & $3889(7)$ & $454(12)$ & 7341(7) & $78(3)$ \\
\hline$C(14)$ & $4533(8)$ & $383(14)$ & 7683(7) & $95(4)$ \\
\hline$C(15)$ & $5204(6)$ & $814(12)$ & 7399(6) & $68(3)$ \\
\hline$C(16)$ & $6768(4)$ & 2151(7) & 7088(4) & $34(1)$ \\
\hline$C(17)$ & $6623(6)$ & 2973(11) & $7647(5)$ & $60(3)$ \\
\hline$C(18)$ & $7119(6)$ & $3015(9)$ & $8213(4)$ & $59(2)$ \\
\hline$C(19)$ & $7757(6)$ & $2250(9)$ & $8220(5)$ & $59(2)$ \\
\hline$C(20)$ & $7890(8)$ & $1450(11)$ & $7680(6)$ & $76(4)$ \\
\hline
\end{tabular}




\begin{tabular}{lllll}
$\mathrm{C}(21)$ & $7414(6)$ & $1391(8)$ & $7099(5)$ & $57(2)$ \\
$\mathrm{C}(22)$ & $6141(5)$ & $3959(7)$ & $4313(5)$ & $37(2)$ \\
$\mathrm{C}(23)$ & $5933(5)$ & $3157(8)$ & $3756(4)$ & $46(2)$ \\
$\mathrm{C}(24)$ & $5482(6)$ & $3606(10)$ & $3216(5)$ & $58(2)$ \\
$\mathrm{C}(25)$ & $5202(5)$ & $4834(11)$ & $3235(5)$ & $67(3)$ \\
$\mathrm{C}(26)$ & $5385(6)$ & $5652(10)$ & $3781(6)$ & $64(3)$ \\
$\mathrm{C}(27)$ & $5867(5)$ & $5207(8)$ & $4324(5)$ & $54(2)$ \\
$\mathrm{C}(28)$ & $7744(4)$ & $3553(8)$ & $4747(4)$ & $38(2)$ \\
$\mathrm{C}(29)$ & $8264(5)$ & $2543(10)$ & $4783(5)$ & $54(2)$ \\
$\mathrm{C}(30)$ & $9040(5)$ & $2755(10)$ & $4613(5)$ & $61(3)$ \\
$\mathrm{C}(31)$ & $9281(7)$ & $3941(10)$ & $4387(6)$ & $62(3)$ \\
$\mathrm{C}(32)$ & $8759(5)$ & $4934(10)$ & $4361(6)$ & $61(2)$ \\
$\mathrm{C}(33)$ & $7989(5)$ & $4788(8)$ & $4520(5)$ & $52(2)$ \\
$\mathrm{C}(1 \mathrm{~S})$ & $6977(12)$ & $6892(13)$ & $7936(6)$ & $147(9)$ \\
$\mathrm{Cl}(1 \mathrm{~S})$ & $6346(5)$ & $6621(7)$ & $7113(3)$ & $224(5)$ \\
$\mathrm{Cl}(2 \mathrm{~S})$ & $7259(5)$ & $8492(7)$ & $7816(3)$ & $186(3)$ \\
& & & & \\
\hline
\end{tabular}


Table 3. Bond lengths $[\AA]$ and angles $[\mathrm{deg}]$ for $\mathrm{Pt}(\mathrm{dppe})\left(\mathrm{CH}(\mathrm{Me}) \mathrm{CO}_{2} \mathrm{t}-\mathrm{Bu}\right)(\mathrm{Cl}) \cdot \mathrm{CH}_{2} \mathrm{Cl}_{2}$, glu167a.

\begin{tabular}{|c|c|c|c|}
\hline $\operatorname{Pt}(1)-C(1)$ & $2.126(8)$ & $C(19)-C(20)$ & $1.337(14)$ \\
\hline $\operatorname{Pt}(1)-\mathrm{P}(1)$ & $2.2105(19)$ & $C(20)-C(21)$ & $1.381(13)$ \\
\hline $\mathrm{Pt}(1)-\mathrm{P}(2)$ & $2.2872(18)$ & $C(22)-C(27)$ & $1.368(11)$ \\
\hline $\mathrm{Pt}(1)-\mathrm{Cl}(1)$ & $2.361(2)$ & $C(22)-C(23)$ & $1.391(12)$ \\
\hline $\mathrm{P}(1)-\mathrm{C}(10)$ & $1.804(8)$ & $C(23)-C(24)$ & $1.371(12)$ \\
\hline $\mathrm{P}(1)-\mathrm{C}(16)$ & $1.814(7)$ & $C(24)-C(25)$ & $1.353(14)$ \\
\hline $\mathrm{P}(1)-\mathrm{C}(8)$ & $1.833(7)$ & $C(25)-C(26)$ & $1.375(16)$ \\
\hline $\mathrm{P}(2)-\mathrm{C}(28)$ & $1.821(8)$ & $C(26)-C(27)$ & $1.406(13)$ \\
\hline $\mathrm{P}(2)-\mathrm{C}(9)$ & $1.823(7)$ & $C(28)-C(29)$ & $1.376(11)$ \\
\hline $\mathrm{P}(2)-\mathrm{C}(22)$ & $1.835(8)$ & $C(28)-C(33)$ & $1.406(11)$ \\
\hline $\mathrm{O}(1)-\mathrm{C}(2)$ & $1.383(10)$ & $C(29)-C(30)$ & $1.399(13)$ \\
\hline $\mathrm{O}(1)-\mathrm{C}(4)$ & $1.448(10)$ & $\mathrm{C}(30)-\mathrm{C}(31)$ & $1.358(13)$ \\
\hline $\mathrm{O}(2)-\mathrm{C}(2)$ & $1.211(9)$ & $\mathrm{C}(31)-\mathrm{C}(32)$ & $1.364(14)$ \\
\hline $\mathrm{C}(1)-\mathrm{C}(2)$ & $1.486(11)$ & $C(32)-C(33)$ & $1.376(12)$ \\
\hline$C(1)-C(3)$ & $1.539(12)$ & $\mathrm{C}(1 \mathrm{~S})-\mathrm{Cl}(2 \mathrm{~S})$ & $1.731(13)$ \\
\hline$C(4)-C(5)$ & $1.469(14)$ & $\mathrm{C}(1 \mathrm{~S})-\mathrm{Cl}(1 \mathrm{~S})$ & $1.931(15)$ \\
\hline$C(4)-C(7)$ & $1.487(14)$ & & \\
\hline$C(4)-C(6)$ & $1.570(14)$ & $\mathrm{C}(1)-\mathrm{Pt}(1)-\mathrm{P}(1)$ & $91.7(2)$ \\
\hline $\mathrm{C}(8)-\mathrm{C}(9)$ & $1.545(11)$ & $\mathrm{C}(1)-\mathrm{Pt}(1)-\mathrm{P}(2)$ & $175.6(2)$ \\
\hline $\mathrm{C}(10)-\mathrm{C}(11)$ & $1.351(14)$ & $\mathrm{P}(1)-\mathrm{Pt}(1)-\mathrm{P}(2)$ & $86.01(6)$ \\
\hline$C(10)-C(15)$ & $1.399(14)$ & $\mathrm{C}(1)-\mathrm{Pt}(1)-\mathrm{Cl}(1)$ & $88.0(2)$ \\
\hline$C(11)-C(12)$ & $1.391(16)$ & $\mathrm{P}(1)-\mathrm{Pt}(1)-\mathrm{Cl}(1)$ & $175.79(8)$ \\
\hline$C(12)-C(13)$ & $1.350(19)$ & $\mathrm{P}(2)-\mathrm{Pt}(1)-\mathrm{Cl}(1)$ & $94.58(8)$ \\
\hline$C(13)-C(14)$ & $1.294(18)$ & $C(10)-P(1)-C(16)$ & $104.1(4)$ \\
\hline$C(14)-C(15)$ & $1.354(15)$ & $\mathrm{C}(10)-\mathrm{P}(1)-\mathrm{C}(8)$ & $105.1(4)$ \\
\hline$C(16)-C(21)$ & $1.364(11)$ & $C(16)-P(1)-C(8)$ & $103.8(4)$ \\
\hline$C(16)-C(17)$ & $1.382(11)$ & $\mathrm{C}(10)-\mathrm{P}(1)-\mathrm{Pt}(1)$ & $117.5(3)$ \\
\hline $\mathrm{C}(17)-\mathrm{C}(18)$ & $1.380(13)$ & $\mathrm{C}(16)-\mathrm{P}(1)-\mathrm{Pt}(1)$ & $118.0(2)$ \\
\hline$C(18)-C(19)$ & $1.355(14)$ & $\mathrm{C}(8)-\mathrm{P}(1)-\mathrm{Pt}(1)$ & $106.9(2)$ \\
\hline
\end{tabular}




\begin{tabular}{|c|c|c|c|}
\hline $\mathrm{C}(28)-\mathrm{P}(2)-\mathrm{C}(9)$ & 105.1(3) & $\mathrm{C}(17)-\mathrm{C}(16)-\mathrm{P}(1)$ & $119.6(6)$ \\
\hline C(28)-P(2)-C(22) & $106.3(4)$ & $\mathrm{C}(18)-\mathrm{C}(17)-\mathrm{C}(16)$ & $120.5(9)$ \\
\hline C(9)-P(2)-C(22) & $104.6(4)$ & $\mathrm{C}(17)-\mathrm{C}(18)-\mathrm{C}(19)$ & 119.8(9) \\
\hline $\mathrm{C}(28)-\mathrm{P}(2)-\mathrm{Pt}(1)$ & $114.9(3)$ & $\mathrm{C}(20)-\mathrm{C}(19)-\mathrm{C}(18)$ & 119.3(9) \\
\hline $\mathrm{C}(9)-\mathrm{P}(2)-\mathrm{Pt}(1)$ & $106.7(2)$ & $C(19)-C(20)-C(21)$ & $122.8(11)$ \\
\hline $\mathrm{C}(22)-\mathrm{P}(2)-\mathrm{Pt}(1)$ & $118.0(3)$ & $C(16)-C(21)-C(20)$ & 118.3(9) \\
\hline $\mathrm{C}(2)-\mathrm{O}(1)-\mathrm{C}(4)$ & $120.8(7)$ & $\mathrm{C}(27)-\mathrm{C}(22)-\mathrm{C}(23)$ & $118.6(8)$ \\
\hline $\mathrm{C}(2)-\mathrm{C}(1)-\mathrm{C}(3)$ & $109.8(7)$ & $\mathrm{C}(27)-\mathrm{C}(22)-\mathrm{P}(2)$ & $123.5(7)$ \\
\hline $\mathrm{C}(2)-\mathrm{C}(1)-\mathrm{Pt}(1)$ & $108.0(5)$ & $\mathrm{C}(23)-\mathrm{C}(22)-\mathrm{P}(2)$ & $117.8(6)$ \\
\hline $\mathrm{C}(3)-\mathrm{C}(1)-\mathrm{Pt}(1)$ & $113.3(6)$ & $\mathrm{C}(22)-\mathrm{C}(23)-\mathrm{C}(24)$ & $121.3(8)$ \\
\hline $\mathrm{O}(2)-\mathrm{C}(2)-\mathrm{O}(1)$ & $122.3(8)$ & $\mathrm{C}(25)-\mathrm{C}(24)-\mathrm{C}(23)$ & $119.9(9)$ \\
\hline $\mathrm{O}(2)-\mathrm{C}(2)-\mathrm{C}(1)$ & $127.2(7)$ & $\mathrm{C}(24)-\mathrm{C}(25)-\mathrm{C}(26)$ & $120.6(9)$ \\
\hline $\mathrm{O}(1)-\mathrm{C}(2)-\mathrm{C}(1)$ & $110.6(7)$ & $\mathrm{C}(25)-\mathrm{C}(26)-\mathrm{C}(27)$ & 119.6(9) \\
\hline $\mathrm{O}(1)-\mathrm{C}(4)-\mathrm{C}(5)$ & $113.8(8)$ & $\mathrm{C}(22)-\mathrm{C}(27)-\mathrm{C}(26)$ & $120.0(9)$ \\
\hline $\mathrm{O}(1)-\mathrm{C}(4)-\mathrm{C}(7)$ & $109.4(9)$ & $\mathrm{C}(29)-\mathrm{C}(28)-\mathrm{C}(33)$ & $120.0(8)$ \\
\hline $\mathrm{C}(5)-\mathrm{C}(4)-\mathrm{C}(7)$ & $112.7(11)$ & $\mathrm{C}(29)-\mathrm{C}(28)-\mathrm{P}(2)$ & $118.8(7)$ \\
\hline $\mathrm{O}(1)-\mathrm{C}(4)-\mathrm{C}(6)$ & $100.8(7)$ & $\mathrm{C}(33)-\mathrm{C}(28)-\mathrm{P}(2)$ & $121.2(6)$ \\
\hline$C(5)-C(4)-C(6)$ & $110.8(10)$ & $\mathrm{C}(28)-\mathrm{C}(29)-\mathrm{C}(30)$ & $119.9(9)$ \\
\hline$C(7)-C(4)-C(6)$ & $108.7(10)$ & $\mathrm{C}(31)-\mathrm{C}(30)-\mathrm{C}(29)$ & $120.5(9)$ \\
\hline $\mathrm{C}(9)-\mathrm{C}(8)-\mathrm{P}(1)$ & $106.2(5)$ & $\mathrm{C}(32)-\mathrm{C}(31)-\mathrm{C}(30)$ & $118.7(10)$ \\
\hline $\mathrm{C}(8)-\mathrm{C}(9)-\mathrm{P}(2)$ & $107.9(5)$ & $\mathrm{C}(31)-\mathrm{C}(32)-\mathrm{C}(33)$ & $123.5(9)$ \\
\hline$C(11)-C(10)-C(15)$ & $116.7(9)$ & $\mathrm{C}(32)-\mathrm{C}(33)-\mathrm{C}(28)$ & $117.3(8)$ \\
\hline $\mathrm{C}(11)-\mathrm{C}(10)-\mathrm{P}(1)$ & $120.2(7)$ & $\mathrm{Cl}(2 \mathrm{~S})-\mathrm{C}(1 \mathrm{~S})-\mathrm{Cl}(1 \mathrm{~S})$ & $100.9(7)$ \\
\hline$C(15)-C(10)-P(1)$ & $123.0(7)$ & & \\
\hline$C(10)-C(11)-C(12)$ & $119.4(12)$ & $\begin{array}{l}\text { Symmetry transformations } \\
\text { equivalent atoms: }\end{array}$ & used to \\
\hline$C(13)-C(12)-C(11)$ & $121.4(12)$ & & \\
\hline $\mathrm{C}(14)-\mathrm{C}(13)-\mathrm{C}(12)$ & $119.4(11)$ & & \\
\hline$C(13)-C(14)-C(15)$ & $121.4(12)$ & & \\
\hline $\mathrm{C}(14)-\mathrm{C}(15)-\mathrm{C}(10)$ & $121.4(11)$ & & \\
\hline$C(21)-C(16)-C(17)$ & $119.2(7)$ & & \\
\hline $\mathrm{C}(21)-\mathrm{C}(16)-\mathrm{P}(1)$ & $121.2(6)$ & & \\
\hline
\end{tabular}


Table 4. Anisotropic displacement parameters $\left(\AA^{2} \times 10^{3}\right)$ for $\mathrm{Pt}(\mathrm{dppe})\left(\mathrm{CH}(\mathrm{Me}) \mathrm{CO}_{2} \mathrm{t}-\mathrm{Bu}\right)(\mathrm{Cl}) \cdot \mathrm{CH}_{2} \mathrm{Cl}_{2}$, glu167a. The anisotropic displacement factor exponent takes the form: $-2 \pi^{2}\left[\mathrm{~h}^{2} \mathrm{a}^{* 2} \mathrm{U}^{11}+\ldots+2 \mathrm{~h} \mathrm{k} \mathrm{a} \mathrm{a}^{*} \mathrm{~b}^{*} \mathrm{U}^{12}\right]$

\begin{tabular}{|c|c|c|c|c|c|c|}
\hline & $\mathrm{U}^{11}$ & $\mathrm{U}^{22}$ & $\mathrm{U}^{33}$ & $\mathrm{U}^{23}$ & $\mathrm{U}^{13}$ & $\mathrm{U}^{12}$ \\
\hline $\operatorname{Pt}(1)$ & $33(1)$ & $31(1)$ & $29(1)$ & $-4(1)$ & $-4(1)$ & $4(1)$ \\
\hline $\mathrm{Cl}(1)$ & $90(2)$ & $53(1)$ & $35(1)$ & $-13(1)$ & $-4(1)$ & $19(1)$ \\
\hline $\mathrm{P}(1)$ & $29(1)$ & $36(1)$ & $30(1)$ & $-1(1)$ & $0(1)$ & 2(1) \\
\hline $\mathrm{P}(2)$ & $33(1)$ & $37(1)$ & $29(1)$ & $-2(1)$ & $-1(1)$ & $4(1)$ \\
\hline $\mathrm{O}(1)$ & $37(3)$ & $45(3)$ & $47(3)$ & $-8(2)$ & $1(2)$ & 1(2) \\
\hline $\mathrm{O}(2)$ & $52(3)$ & $31(2)$ & $67(4)$ & $-10(3)$ & $2(3)$ & 1(2) \\
\hline$C(1)$ & $36(4)$ & $33(3)$ & $53(5)$ & $-6(3)$ & $7(4)$ & $-1(3)$ \\
\hline$C(2)$ & $43(4)$ & $47(4)$ & $42(4)$ & $-1(3)$ & $0(3)$ & $-7(3)$ \\
\hline$C(3)$ & $30(3)$ & 41(3) & $116(12)$ & $-12(6)$ & 11(6) & $-6(3)$ \\
\hline$C(4)$ & $41(5)$ & $44(4)$ & $52(5)$ & $-3(4)$ & $-3(4)$ & 6(4) \\
\hline $\mathrm{C}(5)$ & $67(7)$ & $65(6)$ & 81(7) & $31(6)$ & $15(5)$ & $33(5)$ \\
\hline$C(6)$ & $44(5)$ & $75(6)$ & $95(8)$ & $-17(6)$ & $-12(5)$ & $2(5)$ \\
\hline$C(7)$ & $59(6)$ & $62(6)$ & $65(7)$ & $5(5)$ & $16(5)$ & $7(5)$ \\
\hline$C(8)$ & $49(5)$ & $35(4)$ & $31(4)$ & $-8(3)$ & $6(4)$ & $8(3)$ \\
\hline$C(9)$ & $51(4)$ & 24(3) & $30(3)$ & $-6(3)$ & 1(3) & 2(3) \\
\hline$C(10)$ & $35(4)$ & $47(4)$ & $44(4)$ & $-8(3)$ & $7(3)$ & $-3(3)$ \\
\hline $\mathrm{C}(11)$ & $48(6)$ & $74(6)$ & $84(8)$ & $14(6)$ & $-1(6)$ & $-12(5)$ \\
\hline$C(12)$ & $32(5)$ & 99(9) & $123(12)$ & $-14(8)$ & $0(6)$ & $-16(5)$ \\
\hline$C(13)$ & $52(7)$ & $88(8)$ & $94(9)$ & $-12(7)$ & $36(6)$ & $-14(6)$ \\
\hline$C(14)$ & $87(9)$ & $122(11)$ & $77(8)$ & $12(7)$ & $29(7)$ & $-51(8)$ \\
\hline$C(15)$ & $55(6)$ & $87(7)$ & $63(6)$ & $4(5)$ & $0(5)$ & $-17(5)$ \\
\hline$C(16)$ & $29(3)$ & $36(3)$ & $36(4)$ & 1(3) & $-3(3)$ & $-3(3)$ \\
\hline $\mathrm{C}(17)$ & $62(6)$ & $74(6)$ & $45(5)$ & $-20(5)$ & $-7(4)$ & $20(5)$ \\
\hline$C(18)$ & $83(7)$ & $67(5)$ & $28(4)$ & $-5(4)$ & $1(4)$ & $-13(5)$ \\
\hline$C(19)$ & $66(6)$ & $60(5)$ & $50(5)$ & 2(4) & $-25(5)$ & $-7(5)$ \\
\hline$C(20)$ & $81(8)$ & $77(7)$ & $70(7)$ & $-24(6)$ & $-44(6)$ & $17(6)$ \\
\hline
\end{tabular}




\begin{tabular}{lllllll}
$\mathrm{C}(21)$ & $69(6)$ & $52(5)$ & $50(5)$ & $-16(4)$ & $-28(5)$ & $14(4)$ \\
$\mathrm{C}(22)$ & $29(4)$ & $45(4)$ & $38(4)$ & $7(3)$ & $-3(3)$ & $4(3)$ \\
$\mathrm{C}(23)$ & $43(4)$ & $53(4)$ & $43(4)$ & $8(4)$ & $-7(4)$ & $-1(4)$ \\
$\mathrm{C}(24)$ & $51(5)$ & $77(6)$ & $47(5)$ & $9(4)$ & $-14(4)$ & $8(5)$ \\
$\mathrm{C}(25)$ & $44(5)$ & $100(8)$ & $57(6)$ & $31(6)$ & $-7(4)$ & $7(5)$ \\
$\mathrm{C}(26)$ & $57(6)$ & $72(6)$ & $63(6)$ & $16(5)$ & $1(5)$ & $24(5)$ \\
$\mathrm{C}(27)$ & $62(6)$ & $53(4)$ & $47(5)$ & $3(4)$ & $4(4)$ & $17(4)$ \\
$\mathrm{C}(28)$ & $32(4)$ & $52(4)$ & $30(3)$ & $-1(3)$ & $1(3)$ & $-1(3)$ \\
$\mathrm{C}(29)$ & $45(4)$ & $64(5)$ & $52(5)$ & $-2(4)$ & $5(4)$ & $15(4)$ \\
$\mathrm{C}(30)$ & $39(5)$ & $83(6)$ & $61(6)$ & $6(5)$ & $7(4)$ & $21(5)$ \\
$\mathrm{C}(31)$ & $48(6)$ & $82(7)$ & $58(6)$ & $4(5)$ & $4(5)$ & $-2(5)$ \\
$\mathrm{C}(32)$ & $41(5)$ & $65(5)$ & $76(7)$ & $-6(5)$ & $12(5)$ & $-10(4)$ \\
$\mathrm{C}(33)$ & $46(5)$ & $45(4)$ & $64(5)$ & $2(4)$ & $5(4)$ & $6(4)$ \\
$\mathrm{C}(1 \mathrm{~S})$ & $300(30)$ & $95(10)$ & $45(7)$ & $3(7)$ & $51(11)$ & $-51(14)$ \\
$\mathrm{Cl}(1 \mathrm{~S})$ & $368(10)$ & $192(5)$ & $113(4)$ & $-76(4)$ & $110(5)$ & $-197(6)$ \\
$\mathrm{Cl}(2 \mathrm{~S})$ & $221(7)$ & $219(6)$ & $117(4)$ & $-38(4)$ & $-12(5)$ & $-76(6)$ \\
& & & & & & \\
\hline
\end{tabular}


Table 5. Hydrogen coordinates ( $\left.\times 10^{4}\right)$ and isotropic displacement parameters $\left(\AA^{2} \times 10^{3}\right)$ for $\mathrm{Pt}(\mathrm{dppe})\left(\mathrm{CH}(\mathrm{Me}) \mathrm{CO}_{2} \mathrm{t}-\mathrm{Bu}\right)(\mathrm{Cl}) \cdot \mathrm{CH}_{2} \mathrm{Cl}_{2}$, glu167a.

\begin{tabular}{|c|c|c|c|c|}
\hline & $\mathrm{X}$ & $\mathrm{y}$ & $\mathrm{z}$ & $\mathrm{U}(\mathrm{eq})$ \\
\hline $\mathrm{H}(1 \mathrm{~A})$ & 6259 & -663 & 6289 & 49 \\
\hline $\mathrm{H}(3 \mathrm{~A})$ & 5522 & -2234 & 5683 & 94 \\
\hline $\mathrm{H}(3 \mathrm{~B})$ & 5619 & -1451 & 4972 & 94 \\
\hline $\mathrm{H}(3 \mathrm{C})$ & 5126 & -854 & 5594 & 94 \\
\hline $\mathrm{H}(5 \mathrm{~A})$ & 8098 & -3497 & 5841 & 106 \\
\hline $\mathrm{H}(5 \mathrm{~B})$ & 8247 & -2908 & 6598 & 106 \\
\hline $\mathrm{H}(5 \mathrm{C})$ & 8954 & -3244 & 6101 & 106 \\
\hline $\mathrm{H}(6 \mathrm{~A})$ & 8775 & -679 & 6742 & 107 \\
\hline $\mathrm{H}(6 \mathrm{~B})$ & 8864 & 223 & 6073 & 107 \\
\hline $\mathrm{H}(6 \mathrm{C})$ & 9460 & -920 & 6206 & 107 \\
\hline $\mathrm{H}(7 \mathrm{~A})$ & 8321 & -2158 & 4801 & 93 \\
\hline $\mathrm{H}(7 \mathrm{~B})$ & 9176 & -1814 & 5033 & 93 \\
\hline $\mathrm{H}(7 \mathrm{C})$ & 8572 & -686 & 4892 & 93 \\
\hline $\mathrm{H}(8 \mathrm{~A})$ & 5414 & 3870 & 5888 & 46 \\
\hline $\mathrm{H}(8 \mathrm{~B})$ & 5811 & 4281 & 6608 & 46 \\
\hline $\mathrm{H}(9 \mathrm{~A})$ & 7047 & 4318 & 6066 & 42 \\
\hline $\mathrm{H}(9 \mathrm{~B})$ & 6492 & 5210 & 5608 & 42 \\
\hline $\mathrm{H}(11 \mathrm{~A})$ & 4517 & 1949 & 5962 & 83 \\
\hline $\mathrm{H}(12 \mathrm{~A})$ & 3393 & 1184 & 6487 & 102 \\
\hline $\mathrm{H}(13 \mathrm{~A})$ & 3438 & 78 & 7527 & 93 \\
\hline $\mathrm{H}(14 \mathrm{~A})$ & 4537 & 25 & 8137 & 114 \\
\hline $\mathrm{H}(15 \mathrm{~A})$ & 5669 & 681 & 7643 & 82 \\
\hline $\mathrm{H}(17 \mathrm{~A})$ & 6182 & 3507 & 7641 & 72 \\
\hline $\mathrm{H}(18 \mathrm{~A})$ & 7015 & 3572 & 8593 & 71 \\
\hline $\mathrm{H}(19 \mathrm{~A})$ & 8103 & 2283 & 8600 & 70 \\
\hline
\end{tabular}




\begin{tabular}{lrrrr}
$\mathrm{H}(20 \mathrm{~A})$ & 8325 & 905 & 7697 & 92 \\
$\mathrm{H}(21 \mathrm{~A})$ & 7532 & 840 & 6720 & 68 \\
$\mathrm{H}(23 \mathrm{~A})$ & 6105 & 2290 & 3749 & 55 \\
$\mathrm{H}(24 \mathrm{~A})$ & 5367 & 3062 & 2834 & 70 \\
$\mathrm{H}(25 \mathrm{~A})$ & 4880 & 5131 & 2871 & 80 \\
$\mathrm{H}(26 \mathrm{~A})$ & 5189 & 6505 & 3791 & 77 \\
$\mathrm{H}(27 \mathrm{~A})$ & 6001 & 5766 & 4695 & 65 \\
$\mathrm{H}(29 \mathrm{~A})$ & 8098 & 1712 & 4922 & 64 \\
$\mathrm{H}(30 \mathrm{~A})$ & 9398 & 2072 & 4656 & 73 \\
$\mathrm{H}(31 \mathrm{~A})$ & 9797 & 4074 & 4251 & 75 \\
$\mathrm{H}(32 \mathrm{~A})$ & 8936 & 5763 & 4227 & 73 \\
$\mathrm{H}(33 \mathrm{~A})$ & 7640 & 5485 & 4479 & 62 \\
$\mathrm{H}(1 \mathrm{SA})$ & 6678 & 6784 & 8369 & 176 \\
$\mathrm{H}(1 \mathrm{SB})$ & 7422 & 6303 & 7944 & 176 \\
& & & & \\
\hline
\end{tabular}


Table 1. Crystal data and structure refinement for $\mathrm{Pt}(\mathrm{dppe})(\mathrm{Me})\left(\mathrm{CH}(\mathrm{Me}) \mathrm{CO}_{2} \mathrm{t}-\mathrm{Bu}\right) \cdot \mathrm{THF}$, glu168.

Identification code

Empirical formula

Formula weight

Temperature

Wavelength

Crystal system

Space group

Unit cell dimensions

Volume

Z

Density (calculated)

Absorption coefficient

$\mathrm{F}(000)$

Crystal size

Theta range for data collection

Index ranges

Reflections collected

Independent reflections

Completeness to theta $=26.00^{\circ}$

Absorption correction

Max. and min. transmission

Refinement method

Data / restraints / parameters

Goodness-of-fit on $\mathrm{F}^{2}$

Final $\mathrm{R}$ indices [I $>2 \operatorname{sigma}(\mathrm{I})]$

$\mathrm{R}$ indices (all data)

Largest diff. peak and hole glu168

$\mathrm{C}_{38} \mathrm{H}_{48} \mathrm{O}_{3} \mathrm{P}_{2} \mathrm{Pt}$

809.79

100(2) K

$0.71073 \AA$

Monoclinic

$\mathrm{P} 2(1) / \mathrm{n}$

$a=10.2780(4) \AA$

$\alpha=90^{\circ}$.

$\mathrm{b}=17.6054(7) \AA$

$\beta=99.7550(10)^{\circ}$.

$\mathrm{c}=20.0000(8) \AA$

$\gamma=90^{\circ}$.

$3566.6(2) \AA^{3}$

4

$1.508 \mathrm{Mg} / \mathrm{m}^{3}$

$4.058 \mathrm{~mm}^{-1}$

1632

$0.20 \times 0.20 \times 0.05 \mathrm{~mm}^{3}$

1.55 to $26.00^{\circ}$.

$-10 \leq \mathrm{h} \leq 12,-21 \leq \mathrm{k} \leq 21,-24 \leq \mathrm{l} \leq 23$

20199

$6997[\mathrm{R}(\mathrm{int})=0.0259]$

$99.9 \%$

Semi-empirical from equivalents

1.000 and 0.727

Full-matrix least-squares on $\mathrm{F}^{2}$

6997 / 0 / 397

1.038

$\mathrm{R} 1=0.0279, \mathrm{wR} 2=0.0650$

$\mathrm{R} 1=0.0322, \mathrm{wR} 2=0.0670$

1.566 and -0.550 e. $\AA^{-3}$ 
Table 2. Atomic coordinates ( $\left.\mathrm{x} 10^{4}\right)$ and equivalent isotropic displacement parameters $\left(\AA^{2} \mathrm{x} 10^{3}\right)$ for $\mathrm{Pt}(\mathrm{dppe})(\mathrm{Me})\left(\mathrm{CH}(\mathrm{Me}) \mathrm{CO}_{2} \mathrm{t}-\mathrm{Bu}\right) \cdot \mathrm{THF}$, glu168. U(eq) is defined as one third of the trace of the orthogonalized $\mathrm{U}^{\mathrm{ij}}$ tensor.

\begin{tabular}{|c|c|c|c|c|}
\hline & $\mathrm{x}$ & $\mathrm{y}$ & $\mathrm{z}$ & $\mathrm{U}(\mathrm{eq})$ \\
\hline $\operatorname{Pt}(1)$ & $6288(1)$ & $1829(1)$ & 1109(1) & $15(1)$ \\
\hline $\mathrm{P}(1)$ & $8278(1)$ & 2123(1) & $862(1)$ & $16(1)$ \\
\hline $\mathrm{P}(2)$ & $7407(1)$ & $1170(1)$ & 1993(1) & $17(1)$ \\
\hline $\mathrm{O}(1)$ & $2630(2)$ & $2463(2)$ & $996(1)$ & $27(1)$ \\
\hline $\mathrm{O}(2)$ & $4346(2)$ & 2873(1) & 1774(1) & $23(1)$ \\
\hline $\mathrm{C}(1)$ & $5235(3)$ & $2333(2)$ & $206(2)$ & $17(1)$ \\
\hline $\mathrm{C}(2)$ & 4414(4) & $1608(2)$ & $1410(2)$ & $21(1)$ \\
\hline$C(3)$ & $3681(4)$ & $2334(2)$ & $1360(2)$ & $21(1)$ \\
\hline$C(4)$ & 3611(4) & $970(2)$ & 1004(2) & $25(1)$ \\
\hline$C(5)$ & $3900(4)$ & $3666(2)$ & 1783(2) & $26(1)$ \\
\hline$C(6)$ & $5035(4)$ & $4044(2)$ & $2250(2)$ & $33(1)$ \\
\hline$C(7)$ & 2636(4) & $3708(2)$ & 2083(2) & $32(1)$ \\
\hline $\mathrm{C}(8)$ & $3735(4)$ & 4019(2) & 1081(2) & $29(1)$ \\
\hline $\mathrm{C}(9)$ & $8555(4)$ & $3130(2)$ & $732(2)$ & 19(1) \\
\hline$C(10)$ & $7535(4)$ & $3629(2)$ & $801(2)$ & $23(1)$ \\
\hline$C(11)$ & 7715(4) & $4407(2)$ & $763(2)$ & $26(1)$ \\
\hline$C(12)$ & $8920(4)$ & 4698(2) & $670(2)$ & $29(1)$ \\
\hline$C(13)$ & 9940(4) & $4207(2)$ & $606(2)$ & $31(1)$ \\
\hline$C(14)$ & 9764(4) & $3426(2)$ & $632(2)$ & $26(1)$ \\
\hline$C(15)$ & 8797(4) & 1592(2) & $169(2)$ & 19(1) \\
\hline$C(16)$ & 7812(4) & $1254(2)$ & $-308(2)$ & $24(1)$ \\
\hline$C(17)$ & $8153(4)$ & $832(2)$ & $-837(2)$ & $31(1)$ \\
\hline$C(18)$ & $9457(4)$ & $729(2)$ & $-888(2)$ & $31(1)$ \\
\hline$C(19)$ & $10438(4)$ & 1051(2) & $-416(2)$ & $29(1)$ \\
\hline$C(20)$ & $10115(4)$ & $1486(2)$ & 111(2) & $25(1)$ \\
\hline$C(21)$ & $9520(4)$ & $1890(2)$ & $1609(2)$ & $20(1)$ \\
\hline
\end{tabular}




\begin{tabular}{lllll}
$\mathrm{C}(22)$ & $9156(3)$ & $1125(2)$ & $1892(2)$ & $20(1)$ \\
$\mathrm{C}(23)$ & $7445(3)$ & $1504(2)$ & $2859(2)$ & $18(1)$ \\
$\mathrm{C}(24)$ & $6617(4)$ & $2094(2)$ & $2968(2)$ & $23(1)$ \\
$\mathrm{C}(25)$ & $6561(4)$ & $2335(2)$ & $3628(2)$ & $28(1)$ \\
$\mathrm{C}(26)$ & $7356(4)$ & $2000(2)$ & $4175(2)$ & $25(1)$ \\
$\mathrm{C}(27)$ & $8215(4)$ & $1421(2)$ & $4064(2)$ & $25(1)$ \\
$\mathrm{C}(28)$ & $8260(4)$ & $1172(2)$ & $3414(2)$ & $23(1)$ \\
$\mathrm{C}(29)$ & $6858(3)$ & $185(2)$ & $1993(2)$ & $20(1)$ \\
$\mathrm{C}(30)$ & $6284(4)$ & $-124(2)$ & $2509(2)$ & $23(1)$ \\
$\mathrm{C}(31)$ & $5790(4)$ & $-859(2)$ & $2455(2)$ & $30(1)$ \\
$\mathrm{C}(32)$ & $5860(4)$ & $-1286(2)$ & $1881(2)$ & $33(1)$ \\
$\mathrm{C}(33)$ & $6413(4)$ & $-977(2)$ & $1360(2)$ & $33(1)$ \\
$\mathrm{C}(34)$ & $6905(4)$ & $-245(2)$ & $1412(2)$ & $27(1)$ \\
$\mathrm{O}(1 \mathrm{~S})$ & $3245(4)$ & $5799(2)$ & $1718(2)$ & $53(1)$ \\
$\mathrm{C}(1 \mathrm{~S})$ & $2322(5)$ & $6035(3)$ & $2134(2)$ & $43(1)$ \\
$\mathrm{C}(2 \mathrm{~S})$ & $2149(6)$ & $6851(3)$ & $2011(3)$ & $60(2)$ \\
$\mathrm{C}(3 \mathrm{~S})$ & $2281(10)$ & $6915(4)$ & $1272(4)$ & $105(3)$ \\
$\mathrm{C}(4 \mathrm{~S})$ & $2970(6)$ & $6250(3)$ & $1114(2)$ & $54(1)$ \\
\hline
\end{tabular}


Table 3. Bond lengths $[\AA]$ and angles $[\mathrm{deg}]$ for $\mathrm{Pt}(\mathrm{dppe})(\mathrm{Me})\left(\mathrm{CH}(\mathrm{Me}) \mathrm{CO}_{2} \mathrm{t}-\mathrm{Bu}\right) \cdot \mathrm{THF}$, glu168.

\begin{tabular}{|c|c|c|c|}
\hline $\operatorname{Pt}(1)-C(1)$ & $2.135(3)$ & $C(19)-C(20)$ & $1.388(5)$ \\
\hline $\mathrm{Pt}(1)-\mathrm{C}(2)$ & $2.149(4)$ & $C(21)-C(22)$ & $1.531(5)$ \\
\hline $\mathrm{Pt}(1)-\mathrm{P}(1)$ & $2.2432(9)$ & $C(23)-C(24)$ & $1.383(5)$ \\
\hline $\mathrm{Pt}(1)-\mathrm{P}(2)$ & $2.2604(9)$ & $C(23)-C(28)$ & $1.401(5)$ \\
\hline $\mathrm{P}(1)-\mathrm{C}(9)$ & $1.822(4)$ & $C(24)-C(25)$ & $1.398(5)$ \\
\hline $\mathrm{P}(1)-\mathrm{C}(15)$ & $1.825(4)$ & $C(25)-C(26)$ & $1.381(5)$ \\
\hline $\mathrm{P}(1)-\mathrm{C}(21)$ & $1.839(4)$ & $C(26)-C(27)$ & $1.391(6)$ \\
\hline $\mathrm{P}(2)-\mathrm{C}(23)$ & $1.822(3)$ & $\mathrm{C}(27)-\mathrm{C}(28)$ & $1.380(5)$ \\
\hline $\mathrm{P}(2)-\mathrm{C}(29)$ & $1.823(4)$ & $C(29)-C(30)$ & $1.385(5)$ \\
\hline $\mathrm{P}(2)-\mathrm{C}(22)$ & $1.844(4)$ & $C(29)-C(34)$ & $1.394(5)$ \\
\hline $\mathrm{O}(1)-\mathrm{C}(3)$ & $1.218(4)$ & $\mathrm{C}(30)-\mathrm{C}(31)$ & $1.388(5)$ \\
\hline $\mathrm{O}(2)-\mathrm{C}(3)$ & $1.364(4)$ & $\mathrm{C}(31)-\mathrm{C}(32)$ & $1.384(6)$ \\
\hline $\mathrm{O}(2)-\mathrm{C}(5)$ & $1.470(4)$ & $\mathrm{C}(32)-\mathrm{C}(33)$ & $1.381(6)$ \\
\hline$C(2)-C(3)$ & $1.478(5)$ & $\mathrm{C}(33)-\mathrm{C}(34)$ & $1.382(5)$ \\
\hline$C(2)-C(4)$ & $1.540(5)$ & $\mathrm{O}(1 \mathrm{~S})-\mathrm{C}(1 \mathrm{~S})$ & $1.425(5)$ \\
\hline$C(5)-C(6)$ & $1.518(5)$ & $\mathrm{O}(1 \mathrm{~S})-\mathrm{C}(4 \mathrm{~S})$ & $1.434(6)$ \\
\hline$C(5)-C(8)$ & $1.519(5)$ & $\mathrm{C}(1 \mathrm{~S})-\mathrm{C}(2 \mathrm{~S})$ & $1.464(7)$ \\
\hline $\mathrm{C}(5)-\mathrm{C}(7)$ & $1.523(5)$ & $\mathrm{C}(2 \mathrm{~S})-\mathrm{C}(3 \mathrm{~S})$ & $1.510(8)$ \\
\hline$C(9)-C(14)$ & $1.393(5)$ & $\mathrm{C}(3 \mathrm{~S})-\mathrm{C}(4 \mathrm{~S})$ & $1.432(8)$ \\
\hline $\mathrm{C}(9)-\mathrm{C}(10)$ & $1.393(5)$ & & \\
\hline$C(10)-C(11)$ & $1.386(5)$ & $C(1)-\operatorname{Pt}(1)-C(2)$ & $87.82(14)$ \\
\hline$C(11)-C(12)$ & $1.381(6)$ & $\mathrm{C}(1)-\mathrm{Pt}(1)-\mathrm{P}(1)$ & $93.95(9)$ \\
\hline$C(12)-C(13)$ & $1.381(6)$ & $\mathrm{C}(2)-\mathrm{Pt}(1)-\mathrm{P}(1)$ & $175.59(10)$ \\
\hline$C(13)-C(14)$ & $1.389(5)$ & $\mathrm{C}(1)-\mathrm{Pt}(1)-\mathrm{P}(2)$ & 173.30(9) \\
\hline$C(15)-C(20)$ & $1.392(5)$ & $\mathrm{C}(2)-\mathrm{Pt}(1)-\mathrm{P}(2)$ & $92.80(10)$ \\
\hline$C(15)-C(16)$ & $1.401(5)$ & $P(1)-P t(1)-P(2)$ & $85.91(3)$ \\
\hline$C(16)-C(17)$ & $1.385(5)$ & $\mathrm{C}(9)-\mathrm{P}(1)-\mathrm{C}(15)$ & $108.49(16)$ \\
\hline $\mathrm{C}(17)-\mathrm{C}(18)$ & $1.373(6)$ & $\mathrm{C}(9)-\mathrm{P}(1)-\mathrm{C}(21)$ & $103.24(16)$ \\
\hline $\mathrm{C}(18)-\mathrm{C}(19)$ & $1.380(6)$ & $\mathrm{C}(15)-\mathrm{P}(1)-\mathrm{C}(21)$ & $104.50(16)$ \\
\hline
\end{tabular}




\begin{tabular}{|c|c|}
\hline $\mathrm{C}(9)-\mathrm{P}(1)-\mathrm{Pt}(1)$ & $115.24(13)$ \\
\hline C(15)-P(1)-Pt(1) & $116.34(12)$ \\
\hline $\mathrm{C}(21)-\mathrm{P}(1)-\mathrm{Pt}(1)$ & $107.65(12)$ \\
\hline C(23)-P(2)-C(29) & $105.33(16)$ \\
\hline $\mathrm{C}(23)-\mathrm{P}(2)-\mathrm{C}(22)$ & $104.71(16)$ \\
\hline $\mathrm{C}(29)-\mathrm{P}(2)-\mathrm{C}(22)$ & $105.45(16)$ \\
\hline $\mathrm{C}(23)-\mathrm{P}(2)-\mathrm{Pt}(1)$ & $120.24(12)$ \\
\hline C(29)-P(2)-Pt(1) & $111.82(11)$ \\
\hline $\mathrm{C}(22)-\mathrm{P}(2)-\mathrm{Pt}(1)$ & $108.18(11)$ \\
\hline $\mathrm{C}(3)-\mathrm{O}(2)-\mathrm{C}(5)$ & $122.9(3)$ \\
\hline$C(3)-C(2)-C(4)$ & $111.9(3)$ \\
\hline$C(3)-C(2)-P t(1)$ & $107.2(2)$ \\
\hline $\mathrm{C}(4)-\mathrm{C}(2)-\mathrm{Pt}(1)$ & $114.2(2)$ \\
\hline $\mathrm{O}(1)-\mathrm{C}(3)-\mathrm{O}(2)$ & $122.4(3)$ \\
\hline $\mathrm{O}(1)-\mathrm{C}(3)-\mathrm{C}(2)$ & $126.3(3)$ \\
\hline $\mathrm{O}(2)-\mathrm{C}(3)-\mathrm{C}(2)$ & $111.3(3)$ \\
\hline $\mathrm{O}(2)-\mathrm{C}(5)-\mathrm{C}(6)$ & $102.5(3)$ \\
\hline $\mathrm{O}(2)-\mathrm{C}(5)-\mathrm{C}(8)$ & $111.4(3)$ \\
\hline$C(6)-C(5)-C(8)$ & $110.0(3)$ \\
\hline $\mathrm{O}(2)-\mathrm{C}(5)-\mathrm{C}(7)$ & $109.8(3)$ \\
\hline$C(6)-C(5)-C(7)$ & $110.7(3)$ \\
\hline $\mathrm{C}(8)-\mathrm{C}(5)-\mathrm{C}(7)$ & $112.0(3)$ \\
\hline C(14)-C(9)-C(10) & $118.9(3)$ \\
\hline $\mathrm{C}(14)-\mathrm{C}(9)-\mathrm{P}(1)$ & $123.3(3)$ \\
\hline $\mathrm{C}(10)-\mathrm{C}(9)-\mathrm{P}(1)$ & $117.5(3)$ \\
\hline $\mathrm{C}(11)-\mathrm{C}(10)-\mathrm{C}(9)$ & $120.5(4)$ \\
\hline $\mathrm{C}(12)-\mathrm{C}(11)-\mathrm{C}(10)$ & $120.3(4)$ \\
\hline $\mathrm{C}(13)-\mathrm{C}(12)-\mathrm{C}(11)$ & $119.5(4)$ \\
\hline $\mathrm{C}(12)-\mathrm{C}(13)-\mathrm{C}(14)$ & $120.7(4)$ \\
\hline C(13)-C(14)-C(9) & $120.0(4)$ \\
\hline$C(20)-C(15)-C(16)$ & $119.2(3)$ \\
\hline
\end{tabular}

\begin{tabular}{|c|c|}
\hline $\mathrm{C}(20)-\mathrm{C}(15)-\mathrm{P}(1)$ & $123.1(3)$ \\
\hline $\mathrm{C}(16)-\mathrm{C}(15)-\mathrm{P}(1)$ & $117.7(3)$ \\
\hline$C(17)-C(16)-C(15)$ & $120.1(4)$ \\
\hline $\mathrm{C}(18)-\mathrm{C}(17)-\mathrm{C}(16)$ & $120.2(4)$ \\
\hline$C(17)-C(18)-C(19)$ & $120.3(4)$ \\
\hline $\mathrm{C}(18)-\mathrm{C}(19)-\mathrm{C}(20)$ & $120.3(4)$ \\
\hline$C(19)-C(20)-C(15)$ & $120.0(4)$ \\
\hline $\mathrm{C}(22)-\mathrm{C}(21)-\mathrm{P}(1)$ & $108.5(2)$ \\
\hline $\mathrm{C}(21)-\mathrm{C}(22)-\mathrm{P}(2)$ & $107.8(2)$ \\
\hline $\mathrm{C}(24)-\mathrm{C}(23)-\mathrm{C}(28)$ & 119.3(3) \\
\hline $\mathrm{C}(24)-\mathrm{C}(23)-\mathrm{P}(2)$ & $118.5(3)$ \\
\hline $\mathrm{C}(28)-\mathrm{C}(23)-\mathrm{P}(2)$ & $122.2(3)$ \\
\hline $\mathrm{C}(23)-\mathrm{C}(24)-\mathrm{C}(25)$ & $120.2(3)$ \\
\hline$C(26)-C(25)-C(24)$ & $120.3(4)$ \\
\hline$C(25)-C(26)-C(27)$ & $119.5(3)$ \\
\hline $\mathrm{C}(28)-\mathrm{C}(27)-\mathrm{C}(26)$ & $120.4(3)$ \\
\hline $\mathrm{C}(27)-\mathrm{C}(28)-\mathrm{C}(23)$ & $120.3(3)$ \\
\hline $\mathrm{C}(30)-\mathrm{C}(29)-\mathrm{C}(34)$ & 119.1(3) \\
\hline $\mathrm{C}(30)-\mathrm{C}(29)-\mathrm{P}(2)$ & $123.0(3)$ \\
\hline $\mathrm{C}(34)-\mathrm{C}(29)-\mathrm{P}(2)$ & $117.6(3)$ \\
\hline $\mathrm{C}(29)-\mathrm{C}(30)-\mathrm{C}(31)$ & $120.4(3)$ \\
\hline $\mathrm{C}(32)-\mathrm{C}(31)-\mathrm{C}(30)$ & $120.0(4)$ \\
\hline $\mathrm{C}(33)-\mathrm{C}(32)-\mathrm{C}(31)$ & $119.9(4)$ \\
\hline $\mathrm{C}(32)-\mathrm{C}(33)-\mathrm{C}(34)$ & $120.2(4)$ \\
\hline C(33)-C(34)-C(29) & $120.4(4)$ \\
\hline $\mathrm{C}(1 \mathrm{~S})-\mathrm{O}(1 \mathrm{~S})-\mathrm{C}(4 \mathrm{~S})$ & $105.9(3)$ \\
\hline $\mathrm{O}(1 \mathrm{~S})-\mathrm{C}(1 \mathrm{~S})-\mathrm{C}(2 \mathrm{~S})$ & $105.2(4)$ \\
\hline $\mathrm{C}(1 \mathrm{~S})-\mathrm{C}(2 \mathrm{~S})-\mathrm{C}(3 \mathrm{~S})$ & $102.0(5)$ \\
\hline $\mathrm{C}(4 \mathrm{~S})-\mathrm{C}(3 \mathrm{~S})-\mathrm{C}(2 \mathrm{~S})$ & $106.6(5)$ \\
\hline $\mathrm{C}(3 \mathrm{~S})-\mathrm{C}(4 \mathrm{~S})-\mathrm{O}(1 \mathrm{~S})$ & $107.5(4)$ \\
\hline
\end{tabular}

Symmetry transformations used to generate equivalent atoms: 
Table 4. Anisotropic displacement parameters $\left(\AA^{2} \times 10^{3}\right)$ for $\mathrm{Pt}(\mathrm{dppe})(\mathrm{Me})\left(\mathrm{CH}(\mathrm{Me}) \mathrm{CO}_{2} \mathrm{t}-\mathrm{Bu}\right) \cdot \mathrm{THF}$, glu168. The anisotropic displacement factor exponent takes the form: $-2 \pi^{2}\left[\mathrm{~h}^{2} \mathrm{a}^{* 2} \mathrm{U}^{11}+\ldots+2 \mathrm{~h} \mathrm{k} \mathrm{a} \mathrm{b}^{*}\right.$ $\mathrm{U}^{12}$ ]

\begin{tabular}{|c|c|c|c|c|c|c|}
\hline & $\mathrm{U}^{11}$ & $\mathrm{U}^{22}$ & $\mathrm{U}^{33}$ & $\mathrm{U}^{23}$ & $\mathrm{U}^{13}$ & $\mathrm{U}^{12}$ \\
\hline $\operatorname{Pt}(1)$ & $16(1)$ & $15(1)$ & $15(1)$ & $0(1)$ & $5(1)$ & $0(1)$ \\
\hline $\mathrm{P}(1)$ & $18(1)$ & $16(1)$ & $16(1)$ & 1(1) & $5(1)$ & $0(1)$ \\
\hline $\mathrm{P}(2)$ & $20(1)$ & $15(1)$ & $16(1)$ & $0(1)$ & $5(1)$ & $1(1)$ \\
\hline $\mathrm{O}(1)$ & $19(1)$ & $26(1)$ & $35(2)$ & $-6(1)$ & $3(1)$ & $1(1)$ \\
\hline $\mathrm{O}(2)$ & $22(1)$ & $20(1)$ & $27(1)$ & $-4(1)$ & $3(1)$ & $2(1)$ \\
\hline$C(1)$ & $17(2)$ & $17(2)$ & $17(2)$ & $-2(1)$ & $5(1)$ & $0(1)$ \\
\hline$C(2)$ & 21(2) & $19(2)$ & $24(2)$ & $-4(2)$ & $9(2)$ & $-5(2)$ \\
\hline$C(3)$ & $20(2)$ & $24(2)$ & $21(2)$ & $-2(1)$ & $9(2)$ & $-2(2)$ \\
\hline$C(4)$ & $23(2)$ & $16(2)$ & $39(2)$ & $-5(2)$ & $10(2)$ & $-3(2)$ \\
\hline$C(5)$ & $27(2)$ & $20(2)$ & $31(2)$ & $-5(2)$ & $8(2)$ & $4(2)$ \\
\hline$C(6)$ & $38(2)$ & $21(2)$ & $37(2)$ & $-8(2)$ & $1(2)$ & $1(2)$ \\
\hline$C(7)$ & $36(2)$ & $25(2)$ & $36(2)$ & $-4(2)$ & $13(2)$ & $4(2)$ \\
\hline$C(8)$ & $32(2)$ & $24(2)$ & $34(2)$ & $0(2)$ & $10(2)$ & $1(2)$ \\
\hline$C(9)$ & $23(2)$ & $21(2)$ & $15(2)$ & $0(1)$ & $3(1)$ & $1(1)$ \\
\hline$C(10)$ & $26(2)$ & $22(2)$ & $23(2)$ & $-3(2)$ & $9(2)$ & $-1(2)$ \\
\hline$C(11)$ & $32(2)$ & $20(2)$ & $29(2)$ & $-3(2)$ & $9(2)$ & $6(2)$ \\
\hline$C(12)$ & $40(2)$ & $16(2)$ & $33(2)$ & $1(2)$ & $10(2)$ & $-1(2)$ \\
\hline$C(13)$ & $30(2)$ & $25(2)$ & $40(2)$ & $8(2)$ & $12(2)$ & $-6(2)$ \\
\hline$C(14)$ & $23(2)$ & $22(2)$ & $34(2)$ & $6(2)$ & $10(2)$ & $3(2)$ \\
\hline$C(15)$ & $25(2)$ & $15(2)$ & $18(2)$ & $2(1)$ & $10(1)$ & $1(1)$ \\
\hline$C(16)$ & $28(2)$ & $22(2)$ & $25(2)$ & $-3(2)$ & $10(2)$ & $-2(2)$ \\
\hline$C(17)$ & $44(3)$ & $23(2)$ & $26(2)$ & $-7(2)$ & $9(2)$ & $-7(2)$ \\
\hline$C(18)$ & $49(3)$ & $21(2)$ & $28(2)$ & $-2(2)$ & $22(2)$ & $1(2)$ \\
\hline$C(19)$ & $31(2)$ & $24(2)$ & $35(2)$ & $-1(2)$ & $18(2)$ & $3(2)$ \\
\hline$C(20)$ & $30(2)$ & $20(2)$ & $27(2)$ & $1(2)$ & $12(2)$ & $-1(2)$ \\
\hline $\mathrm{C}(21)$ & $20(2)$ & $22(2)$ & $19(2)$ & $0(1)$ & $6(1)$ & $2(1)$ \\
\hline
\end{tabular}




\begin{tabular}{lllllll}
$\mathrm{C}(22)$ & $20(2)$ & $19(2)$ & $20(2)$ & $3(1)$ & $5(1)$ & $2(1)$ \\
$\mathrm{C}(23)$ & $23(2)$ & $16(2)$ & $16(2)$ & $-1(1)$ & $8(1)$ & $-5(1)$ \\
$\mathrm{C}(24)$ & $26(2)$ & $20(2)$ & $22(2)$ & $-1(2)$ & $3(2)$ & $-1(2)$ \\
$\mathrm{C}(25)$ & $29(2)$ & $25(2)$ & $30(2)$ & $-7(2)$ & $7(2)$ & $2(2)$ \\
$\mathrm{C}(26)$ & $29(2)$ & $27(2)$ & $21(2)$ & $-8(2)$ & $8(2)$ & $-8(2)$ \\
$\mathrm{C}(27)$ & $30(2)$ & $24(2)$ & $20(2)$ & $1(2)$ & $1(2)$ & $-5(2)$ \\
$\mathrm{C}(28)$ & $26(2)$ & $19(2)$ & $22(2)$ & $-2(2)$ & $4(2)$ & $0(2)$ \\
$\mathrm{C}(29)$ & $20(2)$ & $18(2)$ & $22(2)$ & $3(1)$ & $4(1)$ & $3(1)$ \\
$\mathrm{C}(30)$ & $26(2)$ & $25(2)$ & $20(2)$ & $-1(2)$ & $7(2)$ & $-2(2)$ \\
$\mathrm{C}(31)$ & $31(2)$ & $31(2)$ & $31(2)$ & $4(2)$ & $10(2)$ & $-9(2)$ \\
$\mathrm{C}(32)$ & $36(2)$ & $17(2)$ & $44(2)$ & $-2(2)$ & $4(2)$ & $-6(2)$ \\
$\mathrm{C}(33)$ & $40(2)$ & $23(2)$ & $38(2)$ & $-14(2)$ & $9(2)$ & $-3(2)$ \\
$\mathrm{C}(34)$ & $35(2)$ & $22(2)$ & $26(2)$ & $-1(2)$ & $11(2)$ & $0(2)$ \\
$\mathrm{O}(1 \mathrm{~S})$ & $66(2)$ & $55(2)$ & $42(2)$ & $12(2)$ & $22(2)$ & $31(2)$ \\
$\mathrm{C}(1 \mathrm{~S})$ & $47(3)$ & $45(3)$ & $43(3)$ & $9(2)$ & $23(2)$ & $4(2)$ \\
$\mathrm{C}(2 \mathrm{~S})$ & $66(4)$ & $58(4)$ & $61(4)$ & $-2(3)$ & $20(3)$ & $18(3)$ \\
$\mathrm{C}(3 \mathrm{~S})$ & $182(9)$ & $83(5)$ & $68(4)$ & $38(4)$ & $72(5)$ & $72(5)$ \\
$\mathrm{C}(4 \mathrm{~S})$ & $60(3)$ & $68(4)$ & $39(3)$ & $16(2)$ & $18(2)$ & $23(3)$ \\
\hline & & & & & & \\
\hline
\end{tabular}


Table 5. Hydrogen coordinates ( $\left.\times 10^{4}\right)$ and isotropic displacement parameters $\left(\AA^{2} \times 10^{3}\right)$ for $\mathrm{Pt}(\mathrm{dppe})(\mathrm{Me})\left(\mathrm{CH}(\mathrm{Me}) \mathrm{CO}_{2} \mathrm{t}-\mathrm{Bu}\right) \cdot \mathrm{THF}$, glu168.

\begin{tabular}{|c|c|c|c|c|}
\hline & $\mathrm{x}$ & $\mathrm{y}$ & z & $\mathrm{U}(\mathrm{eq})$ \\
\hline $\mathrm{H}(1 \mathrm{~A})$ & 5039 & 2865 & 295 & 25 \\
\hline $\mathrm{H}(1 \mathrm{~B})$ & 4407 & 2057 & 62 & 25 \\
\hline $\mathrm{H}(1 \mathrm{C})$ & 5774 & 2309 & -153 & 25 \\
\hline $\mathrm{H}(2 \mathrm{~A})$ & 4586 & 1452 & 1898 & 25 \\
\hline $\mathrm{H}(4 \mathrm{~A})$ & 2776 & 901 & 1171 & 38 \\
\hline $\mathrm{H}(4 \mathrm{~B})$ & 4117 & 496 & 1060 & 38 \\
\hline $\mathrm{H}(4 \mathrm{C})$ & 3430 & 1108 & 522 & 38 \\
\hline $\mathrm{H}(6 \mathrm{~A})$ & 5838 & 4011 & 2048 & 49 \\
\hline $\mathrm{H}(6 \mathrm{~B})$ & 5178 & 3787 & 2691 & 49 \\
\hline $\mathrm{H}(6 \mathrm{C})$ & 4820 & 4579 & 2311 & 49 \\
\hline $\mathrm{H}(7 \mathrm{~A})$ & 1916 & 3463 & 1775 & 48 \\
\hline $\mathrm{H}(7 \mathrm{~B})$ & 2411 & 4241 & 2146 & 48 \\
\hline $\mathrm{H}(7 \mathrm{C})$ & 2767 & 3447 & 2523 & 48 \\
\hline $\mathrm{H}(8 \mathrm{~A})$ & 4568 & 3981 & 906 & 44 \\
\hline $\mathrm{H}(8 \mathrm{~B})$ & 3490 & 4555 & 1108 & 44 \\
\hline $\mathrm{H}(8 \mathrm{C})$ & 3040 & 3750 & 776 & 44 \\
\hline $\mathrm{H}(10 \mathrm{~A})$ & 6708 & 3435 & 874 & 27 \\
\hline $\mathrm{H}(11 \mathrm{~A})$ & 7007 & 4742 & 801 & 32 \\
\hline $\mathrm{H}(12 \mathrm{~A})$ & 9046 & 5232 & 650 & 35 \\
\hline $\mathrm{H}(13 \mathrm{~A})$ & 10770 & 4406 & 544 & 37 \\
\hline $\mathrm{H}(14 \mathrm{~A})$ & 10469 & 3093 & 581 & 31 \\
\hline $\mathrm{H}(16 \mathrm{~A})$ & 6909 & 1315 & -270 & 29 \\
\hline $\mathrm{H}(17 \mathrm{~A})$ & 7482 & 612 & -1165 & 37 \\
\hline $\mathrm{H}(18 \mathrm{~A})$ & 9685 & 435 & -1249 & 37 \\
\hline $\mathrm{H}(19 \mathrm{~A})$ & 11339 & 975 & -453 & 34 \\
\hline
\end{tabular}




\begin{tabular}{|c|c|c|c|c|}
\hline $\mathrm{H}(20 \mathrm{~A})$ & 10792 & 1710 & 433 & 30 \\
\hline $\mathrm{H}(21 \mathrm{~A})$ & 10406 & 1859 & 1479 & 24 \\
\hline $\mathrm{H}(21 \mathrm{~B})$ & 9539 & 2290 & 1958 & 24 \\
\hline $\mathrm{H}(22 \mathrm{~A})$ & 9721 & 1025 & 2335 & 24 \\
\hline $\mathrm{H}(22 \mathrm{~B})$ & 9289 & 710 & 1576 & 24 \\
\hline $\mathrm{H}(24 \mathrm{~A})$ & 6085 & 2335 & 2593 & 27 \\
\hline $\mathrm{H}(25 \mathrm{~A})$ & 5975 & 2731 & 3701 & 33 \\
\hline $\mathrm{H}(26 \mathrm{~A})$ & 7315 & 2163 & 4624 & 30 \\
\hline $\mathrm{H}(27 \mathrm{~A})$ & 8776 & 1196 & 4438 & 30 \\
\hline $\mathrm{H}(28 \mathrm{~A})$ & 8846 & 775 & 3343 & 27 \\
\hline $\mathrm{H}(30 \mathrm{~A})$ & 6228 & 168 & 2903 & 28 \\
\hline $\mathrm{H}(31 \mathrm{~A})$ & 5404 & -1070 & 2813 & 36 \\
\hline $\mathrm{H}(32 \mathrm{~A})$ & 5527 & -1790 & 1845 & 39 \\
\hline $\mathrm{H}(33 \mathrm{~A})$ & 6455 & -1268 & 964 & 40 \\
\hline $\mathrm{H}(34 \mathrm{~A})$ & 7278 & -34 & 1050 & 33 \\
\hline $\mathrm{H}(1 \mathrm{SA})$ & 2669 & 5935 & 2618 & 52 \\
\hline $\mathrm{H}(1 \mathrm{SB})$ & 1472 & 5764 & 2006 & 52 \\
\hline $\mathrm{H}(2 \mathrm{SA})$ & 2841 & 7148 & 2303 & 72 \\
\hline $\mathrm{H}(2 \mathrm{SB})$ & 1270 & 7025 & 2087 & 72 \\
\hline $\mathrm{H}(3 \mathrm{SA})$ & 1398 & 6941 & 983 & 126 \\
\hline $\mathrm{H}(3 \mathrm{SB})$ & 2782 & 7377 & 1195 & 126 \\
\hline $\mathrm{H}(4 \mathrm{SA})$ & 3803 & 6393 & 960 & 65 \\
\hline $\mathrm{H}(4 \mathrm{SB})$ & 2420 & 5959 & 747 & 65 \\
\hline
\end{tabular}


Table S3. Selected Bond Lengths $(\AA)$ and Bond Angles (deg) for Pt(S,SDiop $)(\mathrm{Cl})\left(\mathrm{CH}(\mathrm{Me})\left(\mathrm{CO}_{2} \mathrm{Et}\right) \quad(20\right.$ - Diop-Et $), \quad \mathrm{Pt}(\mathrm{dppe})(\mathrm{Cl})\left(\mathrm{CH}(\mathrm{Me})\left(\mathrm{CO}_{2} t-\mathrm{Bu}\right)\right)$ $\left(\mathbf{2 0} \bullet \mathbf{C H}_{2} \mathbf{C l}_{2}\right)$, and $\mathrm{Pt}(\mathrm{dppe})(\mathrm{Me})\left(\mathrm{CH}(\mathrm{Me})\left(\mathrm{CO}_{2} t-\mathrm{Bu}\right)\right)(\mathbf{2 1} \cdot \mathbf{T H F})$.

\begin{tabular}{|c|c|c|c|}
\hline Compound & 20-Diop-Et ${ }^{\mathrm{a},}$, & $20^{\circ} \mathrm{CH}_{2} \mathrm{Cl}_{2}{ }^{\mathrm{a}}$ & $21 \cdot \mathrm{THF}^{\mathrm{b}}$ \\
\hline$\overline{\mathrm{Pt}-\mathrm{C}}$ & $2.171(14)$ & $2.126(8)$ & $2.149(4)$ \\
\hline Pt-X & $2.364(4)$ & $2.361(2)$ & $2.135(3)$ \\
\hline$\overline{\mathrm{Pt}-\mathrm{P}(\operatorname{trans} \mathrm{C})}$ & $2.331(4)$ & $2.2872(18)$ & $2.2432(9)$ \\
\hline $\mathrm{Pt}-\mathrm{P}(\operatorname{trans} \mathrm{X})$ & $2.227(4)$ & $2.2105(19)$ & $2.2604(9)$ \\
\hline P-Pt-P & $100.1(1)$ & $86.01(6)$ & $85.91(3)$ \\
\hline $\mathrm{P}(\operatorname{trans} \mathrm{C})-\mathrm{Pt}-\mathrm{C}$ & $172.6(4)$ & $175.6(2)$ & $175.59(10)$ \\
\hline$\overline{\mathrm{P}(\operatorname{trans} \mathrm{Cl})-\mathrm{Pt}-\mathrm{C}}$ & 87.2(4) & $91.7(2)$ & $92.80(10)$ \\
\hline C-Pt-X & $86.4(4)$ & $88.0(2)$ & $87.82(14)$ \\
\hline$\overline{\mathrm{P}(\operatorname{trans} \mathrm{X})-\mathrm{Pt}-\mathrm{X}}$ & $167.4(2)$ & $175.79(8)$ & $173.30(9)$ \\
\hline$\overline{\mathrm{P}(\operatorname{trans} \mathrm{C})-\mathrm{Pt}-\mathrm{X}}$ & $86.5(1)$ & $94.58(8)$ & $93.95(9)$ \\
\hline
\end{tabular}

$$
{ }^{\mathrm{a}} \mathrm{X}=\mathrm{Cl},{ }^{\mathrm{b}} \mathrm{X}=\mathrm{Me}
$$

Figure S1. ORTEP diagram of $\left[(\mathrm{Pt}(\mathrm{dppe})(\mathrm{Me}))_{2}\left(\mu-\mathrm{PPh}_{2}\right)\right][\mathrm{OH}] \cdot 2.5 \mathrm{C}_{7} \mathrm{H}_{8}$. The anion and solvent molecules are not shown.

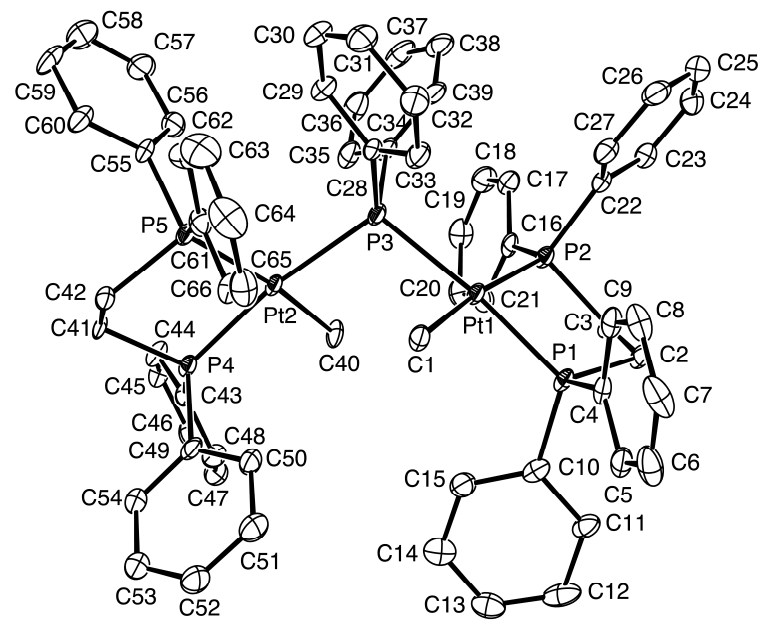


Table 1. Crystal data and structure refinement for $\left[\left(\mathrm{Pt}(\mathrm{dppe})(\mathrm{Me})_{2}\left(\mu-\mathrm{PPh}_{2}\right)\right][\mathrm{OH}] \cdot 2.5 \mathrm{C}_{7} \mathrm{H}_{8}\right.$, glu241.

Identification code

Empirical formula

Formula weight

Temperature

Wavelength

Crystal system

Space group

Unit cell dimensions

Volume

Z

Density (calculated)

Absorption coefficient

$\mathrm{F}(000)$

Crystal size

Theta range for data collection

Index ranges

Reflections collected

Independent reflections

Completeness to theta $=25.00^{\circ}$

Absorption correction

Max. and min. transmission

Refinement method

Data / restraints / parameters

Goodness-of-fit on $\mathrm{F}^{2}$

Final $\mathrm{R}$ indices [I $>2 \operatorname{sigma}(\mathrm{I})]$

$\mathrm{R}$ indices (all data)

Largest diff. peak and hole glu241 CS615

$\mathrm{C}_{83.50} \mathrm{H}_{85} \mathrm{O} \mathrm{P}_{5} \mathrm{Pt}_{2}\left(\mathrm{C}_{66} \mathrm{H}_{64} \mathrm{P}_{5} \mathrm{Pt}, 2.5\left(\mathrm{C}_{7} \mathrm{H}_{8}\right), \mathrm{OH}\right)$

1649.54

$100(2) \mathrm{K}$

$0.71073 \AA$

Triclinic

P-1

$\mathrm{a}=13.0614(12) \AA \quad \alpha=105.3270(10)^{\circ}$.

$\mathrm{b}=16.8404(15) \AA \quad \beta=110.0810(10)^{\circ}$.

$\mathrm{c}=17.8216(16) \AA \quad \gamma=91.9990(10)^{\circ}$.

3516.4(5) $\AA^{3}$

2

$1.558 \mathrm{Mg} / \mathrm{m}^{3}$

$4.135 \mathrm{~mm}^{-1}$

1650

$0.20 \times 0.20 \times 0.10 \mathrm{~mm}^{3}$

1.27 to $28.28^{\circ}$.

$-17<=\mathrm{h}<=16,-21<=\mathrm{k}<=21,-22<=1<=23$

30110

$15539[\mathrm{R}$ (int) $=0.0268]$

$99.1 \%$

multi-scan (sadabs)

0.6826 and 0.4919

Full-matrix least-squares on $\mathrm{F}^{2}$

$15539 / 2 / 683$

1.045

$\mathrm{R} 1=0.0387, \mathrm{wR} 2=0.1057$

$\mathrm{R} 1=0.0474, \mathrm{wR} 2=0.1104$

3.184 and -1.384 e. $\AA^{-3}$ 
Table 2. Atomic coordinates $\left(\mathrm{x} 10^{4}\right)$ and equivalent isotropic displacement parameters $\left(\AA^{2} \times 10^{3}\right)$ for $\left[\left(\mathrm{Pt}(\mathrm{dppe})(\mathrm{Me})_{2}\left(\mu-\mathrm{PPh}_{2}\right)\right][\mathrm{OH}] \cdot 2.5 \mathrm{C}_{7} \mathrm{H}_{8}\right.$, glu241. U(eq) is defined as one third of the trace of the orthogonalized $\mathrm{U}^{\mathrm{ij}}$ tensor.

\begin{tabular}{|c|c|c|c|c|}
\hline & $\mathrm{x}$ & $\mathrm{y}$ & $\mathrm{z}$ & $\mathrm{U}(\mathrm{eq})$ \\
\hline $\operatorname{Pt}(1)$ & $151(1)$ & $6369(1)$ & $2726(1)$ & $24(1)$ \\
\hline $\operatorname{Pt}(2)$ & $-2485(1)$ & $6839(1)$ & $3102(1)$ & $24(1)$ \\
\hline $\mathrm{P}(1)$ & $928(1)$ & $5240(1)$ & $2287(1)$ & $28(1)$ \\
\hline $\mathrm{P}(2)$ & $465(1)$ & $6812(1)$ & $1698(1)$ & $26(1)$ \\
\hline $\mathrm{P}(3)$ & $-729(1)$ & $7438(1)$ & $3276(1)$ & $26(1)$ \\
\hline $\mathrm{P}(4)$ & $-4116(1)$ & $6082(1)$ & $2826(1)$ & $26(1)$ \\
\hline $\mathrm{P}(5)$ & $-2578(1)$ & $7415(1)$ & $4400(1)$ & $27(1)$ \\
\hline$C(1)$ & $-7(4)$ & $5856(3)$ & $3648(3)$ & $31(1)$ \\
\hline$C(2)$ & $1570(4)$ & $5435(3)$ & $1577(3)$ & $33(1)$ \\
\hline$C(3)$ & $812(4)$ & $5897(3)$ & 1027(3) & $31(1)$ \\
\hline$C(4)$ & 2015(4) & 4994(3) & $3114(3)$ & $31(1)$ \\
\hline$C(5)$ & 2157(4) & $4176(3)$ & $3143(3)$ & $38(1)$ \\
\hline$C(6)$ & $2985(5)$ & 4064(4) & $3833(4)$ & $54(2)$ \\
\hline$C(7)$ & $3632(4)$ & $4725(4)$ & $4463(4)$ & $56(2)$ \\
\hline$C(8)$ & $3512(4)$ & $5533(4)$ & $4445(3)$ & $46(1)$ \\
\hline$C(9)$ & 2705(4) & $5659(3)$ & $3766(3)$ & $36(1)$ \\
\hline$C(10)$ & $-42(4)$ & $4293(3)$ & $1702(3)$ & $33(1)$ \\
\hline$C(11)$ & $252(5)$ & $3587(3)$ & $1256(3)$ & $42(1)$ \\
\hline$C(12)$ & $-507(6)$ & 2851(3) & $860(3)$ & $55(2)$ \\
\hline$C(13)$ & $-1551(5)$ & $2845(4)$ & $906(3)$ & $48(1)$ \\
\hline$C(14)$ & $-1846(5)$ & $3538(3)$ & $1335(3)$ & $48(1)$ \\
\hline$C(15)$ & $-1103(4)$ & $4257(3)$ & $1722(3)$ & $39(1)$ \\
\hline$C(16)$ & $-596(4)$ & $7152(3)$ & $912(3)$ & $29(1)$ \\
\hline$C(17)$ & $-535(4)$ & $7965(3)$ & $856(3)$ & $37(1)$ \\
\hline$C(18)$ & $-1356(5)$ & $8178(4)$ & $246(4)$ & $47(1)$ \\
\hline$C(19)$ & $-2244(5)$ & $7602(4)$ & $-309(4)$ & $48(1)$ \\
\hline
\end{tabular}




\begin{tabular}{|c|c|c|c|c|}
\hline$C(20)$ & $-2331(5)$ & 6803(4) & $-252(3)$ & $44(1)$ \\
\hline$C(21)$ & $-1503(4)$ & $6579(3)$ & $359(3)$ & $35(1)$ \\
\hline$C(22)$ & $1702(4)$ & $7550(3)$ & 2066(3) & $31(1)$ \\
\hline$C(23)$ & 2039(4) & 7873(3) & $1519(3)$ & $38(1)$ \\
\hline$C(24)$ & $3015(5)$ & 8394(4) & $1810(4)$ & $48(1)$ \\
\hline$C(25)$ & $3678(5)$ & $8611(4)$ & 2638(4) & $51(2)$ \\
\hline$C(26)$ & $3390(5)$ & 8291(3) & $3190(4)$ & $49(1)$ \\
\hline$C(27)$ & $2385(4)$ & 7761(3) & 2901(3) & $37(1)$ \\
\hline$C(28)$ & $161(4)$ & 8013(3) & 4351(3) & $29(1)$ \\
\hline$C(29)$ & $-125(4)$ & $8760(3)$ & $4749(3)$ & $37(1)$ \\
\hline$C(30)$ & $535(5)$ & 9211(3) & $5563(3)$ & $44(1)$ \\
\hline$C(31)$ & $1472(5)$ & 8941(3) & 5988(3) & $46(1)$ \\
\hline$C(32)$ & 1779(4) & $8209(3)$ & $5592(3)$ & $43(1)$ \\
\hline$C(33)$ & $1123(4)$ & 7758(3) & $4784(3)$ & $37(1)$ \\
\hline$C(34)$ & $-860(4)$ & $8304(3)$ & 2804(3) & $33(1)$ \\
\hline$C(35)$ & $-1851(5)$ & $8436(3)$ & $2295(3)$ & $42(1)$ \\
\hline$C(36)$ & $-1934(6)$ & $9108(4)$ & 1979(4) & $53(2)$ \\
\hline$C(37)$ & $-1011(6)$ & $9648(4)$ & $2165(4)$ & $58(2)$ \\
\hline$C(38)$ & $4(6)$ & 9533(3) & $2677(4)$ & $51(2)$ \\
\hline$C(39)$ & $77(5)$ & $8855(3)$ & 2994(3) & $39(1)$ \\
\hline $\mathrm{C}(40)$ & $-2695(4)$ & $6287(3)$ & $1840(3)$ & $35(1)$ \\
\hline $\mathrm{C}(41)$ & $-4712(4)$ & 6623(3) & $3576(3)$ & $28(1)$ \\
\hline$C(42)$ & $-3784(4)$ & $6828(3)$ & $4430(3)$ & $30(1)$ \\
\hline$C(43)$ & $-5158(4)$ & $5853(3)$ & $1785(3)$ & $28(1)$ \\
\hline$C(44)$ & $-5776(4)$ & 6456(3) & $1552(3)$ & $37(1)$ \\
\hline$C(45)$ & $-6522(4)$ & 6297(3) & $735(3)$ & $41(1)$ \\
\hline$C(46)$ & $-6628(4)$ & $5529(4)$ & $150(3)$ & $40(1)$ \\
\hline $\mathrm{C}(47)$ & $-6012(4)$ & 4935(3) & $378(3)$ & $41(1)$ \\
\hline $\mathrm{C}(48)$ & $-5279(4)$ & $5090(3)$ & 1198(3) & $37(1)$ \\
\hline $\mathrm{C}(49)$ & $-3959(4)$ & 5081(3) & $3017(3)$ & $31(1)$ \\
\hline $\mathrm{C}(50)$ & $-2923(4)$ & $4807(3)$ & $3215(3)$ & $37(1)$ \\
\hline
\end{tabular}




\begin{tabular}{|c|c|c|c|c|}
\hline$C(51)$ & $-2775(5)$ & 4058(3) & $3407(4)$ & $51(1)$ \\
\hline$C(52)$ & $-3669(5)$ & $3584(4)$ & $3397(4)$ & $55(2)$ \\
\hline$C(53)$ & $-4698(5)$ & $3836(4)$ & $3188(4)$ & $52(2)$ \\
\hline$C(54)$ & $-4848(4)$ & $4580(3)$ & $3000(3)$ & $41(1)$ \\
\hline$C(55)$ & $-2941(4)$ & $8468(3)$ & $4576(3)$ & $38(1)$ \\
\hline$C(56)$ & $-3044(4)$ & $8865(3)$ & $3982(4)$ & $39(1)$ \\
\hline$C(57)$ & $-3390(5)$ & $9651(3)$ & 4084(4) & $53(2)$ \\
\hline$C(58)$ & $-3624(6)$ & $10022(4)$ & $4763(5)$ & $74(2)$ \\
\hline$C(59)$ & $-3567(7)$ & $9620(4)$ & $5331(6)$ & $80(2)$ \\
\hline$C(60)$ & $-3213(5)$ & 8839(3) & $5265(4)$ & $58(2)$ \\
\hline$C(61)$ & $-1493(4)$ & $7345(3)$ & $5346(3)$ & $34(1)$ \\
\hline$C(62)$ & $-966(5)$ & 7984(4) & $6058(3)$ & $52(2)$ \\
\hline$C(63)$ & $-170(5)$ & $7850(4)$ & $6731(4)$ & $60(2)$ \\
\hline$C(64)$ & $115(5)$ & $7074(5)$ & $6715(4)$ & $59(2)$ \\
\hline$C(65)$ & $-365(4)$ & 6431(4) & 6017(4) & $48(1)$ \\
\hline$C(66)$ & $-1182(4)$ & 6554(3) & $5315(3)$ & $38(1)$ \\
\hline $\mathrm{O}(1)$ & $4003(5)$ & 7682(3) & $5028(5)$ & $107(3)$ \\
\hline$C(67)$ & $-3054(15)$ & $1159(14)$ & $1471(11)$ & $323(13)$ \\
\hline$C(68)$ & $-3858(7)$ & $1543(6)$ & $999(5)$ & $212(7)$ \\
\hline $\mathrm{C}(69)$ & $-4026(8)$ & $1023(5)$ & $207(7)$ & $163(5)$ \\
\hline$C(70)$ & $-4625(8)$ & $1250(6)$ & $-497(5)$ & $200(7)$ \\
\hline$C(71)$ & $-5056(7)$ & 1998(7) & $-408(5)$ & $139(4)$ \\
\hline$C(72)$ & $-4888(7)$ & $2518(5)$ & $385(6)$ & $180(6)$ \\
\hline$C(73)$ & $-4289(7)$ & 2291(5) & $1089(5)$ & $120(4)$ \\
\hline
\end{tabular}


Table 3. Bond lengths $[\AA]$ and angles $[\mathrm{deg}]$ for $\left[\left(\mathrm{Pt}(\mathrm{dppe})(\mathrm{Me})_{2}\left(\mu-\mathrm{PPh}_{2}\right)\right][\mathrm{OH}] \cdot 2.5 \mathrm{C}_{7} \mathrm{H}_{8}\right.$, glu241.

\begin{tabular}{|c|c|c|c|}
\hline $\operatorname{Pt}(1)-C(1)$ & $2.112(4)$ & $\mathrm{C}(10)-\mathrm{C}(11)$ & $1.391(6)$ \\
\hline $\mathrm{Pt}(1)-\mathrm{P}(1)$ & $2.2701(11)$ & $C(10)-C(15)$ & $1.397(7)$ \\
\hline $\mathrm{Pt}(1)-\mathrm{P}(2)$ & $2.3024(12)$ & $\mathrm{C}(11)-\mathrm{C}(12)$ & $1.411(8)$ \\
\hline $\mathrm{Pt}(1)-\mathrm{P}(3)$ & $2.3470(11)$ & $C(12)-C(13)$ & $1.393(9)$ \\
\hline $\operatorname{Pt}(2)-C(40)$ & $2.109(4)$ & $C(13)-C(14)$ & $1.363(8)$ \\
\hline $\mathrm{Pt}(2)-\mathrm{P}(4)$ & $2.2724(12)$ & $C(14)-C(15)$ & $1.378(7)$ \\
\hline $\mathrm{Pt}(2)-\mathrm{P}(5)$ & $2.3060(11)$ & $C(16)-C(21)$ & $1.386(7)$ \\
\hline $\mathrm{Pt}(2)-\mathrm{P}(3)$ & $2.3584(12)$ & $C(16)-C(17)$ & $1.400(7)$ \\
\hline $\mathrm{P}(1)-\mathrm{C}(4)$ & $1.812(5)$ & $\mathrm{C}(17)-\mathrm{C}(18)$ & $1.376(7)$ \\
\hline$P(1)-C(10)$ & $1.818(5)$ & $\mathrm{C}(18)-\mathrm{C}(19)$ & $1.375(8)$ \\
\hline $\mathrm{P}(1)-\mathrm{C}(2)$ & $1.829(5)$ & $C(19)-C(20)$ & $1.379(8)$ \\
\hline $\mathrm{P}(2)-\mathrm{C}(22)$ & $1.813(5)$ & $C(20)-C(21)$ & $1.391(7)$ \\
\hline $\mathrm{P}(2)-\mathrm{C}(16)$ & $1.831(5)$ & $C(22)-C(27)$ & $1.388(7)$ \\
\hline $\mathrm{P}(2)-\mathrm{C}(3)$ & $1.859(4)$ & $C(22)-C(23)$ & $1.413(7)$ \\
\hline $\mathrm{P}(3)-\mathrm{C}(28)$ & $1.836(5)$ & $C(23)-C(24)$ & $1.376(7)$ \\
\hline $\mathrm{P}(3)-\mathrm{C}(34)$ & $1.851(5)$ & $C(24)-C(25)$ & $1.370(8)$ \\
\hline $\mathrm{P}(4)-C(49)$ & $1.810(5)$ & $C(25)-C(26)$ & $1.386(8)$ \\
\hline $\mathrm{P}(4)-\mathrm{C}(43)$ & $1.815(4)$ & $C(26)-C(27)$ & $1.412(8)$ \\
\hline $\mathrm{P}(4)-\mathrm{C}(41)$ & $1.829(4)$ & $\mathrm{C}(28)-\mathrm{C}(33)$ & $1.381(7)$ \\
\hline $\mathrm{P}(5)-\mathrm{C}(55)$ & $1.826(5)$ & $C(28)-C(29)$ & $1.403(6)$ \\
\hline $\mathrm{P}(5)-\mathrm{C}(61)$ & $1.828(5)$ & $C(29)-C(30)$ & $1.392(7)$ \\
\hline $\mathrm{P}(5)-\mathrm{C}(42)$ & $1.855(5)$ & $\mathrm{C}(30)-\mathrm{C}(31)$ & $1.366(8)$ \\
\hline$C(2)-C(3)$ & $1.528(6)$ & $\mathrm{C}(31)-\mathrm{C}(32)$ & $1.395(8)$ \\
\hline$C(4)-C(9)$ & $1.393(7)$ & $C(32)-C(33)$ & $1.383(7)$ \\
\hline$C(4)-C(5)$ & $1.408(7)$ & $C(34)-C(35)$ & $1.367(7)$ \\
\hline$C(5)-C(6)$ & $1.393(7)$ & $\mathrm{C}(34)-\mathrm{C}(39)$ & $1.393(7)$ \\
\hline$C(6)-C(7)$ & $1.355(9)$ & $C(35)-C(36)$ & $1.382(7)$ \\
\hline$C(7)-C(8)$ & $1.384(9)$ & $C(36)-C(37)$ & $1.371(9)$ \\
\hline $\mathrm{C}(8)-\mathrm{C}(9)$ & $1.377(7)$ & $C(37)-C(38)$ & $1.380(9)$ \\
\hline
\end{tabular}




\begin{tabular}{|c|c|c|c|}
\hline $\mathrm{C}(38)-\mathrm{C}(39)$ & $1.395(7)$ & $C(71)-C(72)$ & 1.3900 \\
\hline $\mathrm{C}(41)-\mathrm{C}(42)$ & $1.526(6)$ & $C(72)-C(73)$ & 1.3900 \\
\hline$C(43)-C(44)$ & $1.381(6)$ & & \\
\hline $\mathrm{C}(43)-\mathrm{C}(48)$ & $1.389(6)$ & $\mathrm{C}(1)-\mathrm{Pt}(1)-\mathrm{P}(1)$ & $87.78(13)$ \\
\hline$C(44)-C(45)$ & $1.393(7)$ & $\mathrm{C}(1)-\mathrm{Pt}(1)-\mathrm{P}(2)$ & $173.05(12)$ \\
\hline$C(45)-C(46)$ & $1.398(7)$ & $\mathrm{P}(1)-\mathrm{Pt}(1)-\mathrm{P}(2)$ & $85.46(4)$ \\
\hline$C(46)-C(47)$ & $1.363(8)$ & $\mathrm{C}(1)-\mathrm{Pt}(1)-\mathrm{P}(3)$ & $85.05(12)$ \\
\hline$C(47)-C(48)$ & $1.393(7)$ & $\mathrm{P}(1)-\mathrm{Pt}(1)-\mathrm{P}(3)$ & $172.59(4)$ \\
\hline $\mathrm{C}(49)-\mathrm{C}(54)$ & $1.398(7)$ & $\mathrm{P}(2)-\mathrm{Pt}(1)-\mathrm{P}(3)$ & $101.75(4)$ \\
\hline$C(49)-C(50)$ & $1.404(6)$ & $\mathrm{C}(40)-\mathrm{Pt}(2)-\mathrm{P}(4)$ & $87.29(13)$ \\
\hline $\mathrm{C}(50)-\mathrm{C}(51)$ & $1.395(7)$ & $\mathrm{C}(40)-\operatorname{Pt}(2)-\mathrm{P}(5)$ & $170.21(13)$ \\
\hline$C(51)-C(52)$ & $1.382(8)$ & $\mathrm{P}(4)-\mathrm{Pt}(2)-\mathrm{P}(5)$ & $84.25(4)$ \\
\hline$C(52)-C(53)$ & $1.380(8)$ & $\mathrm{C}(40)-\mathrm{Pt}(2)-\mathrm{P}(3)$ & $86.60(13)$ \\
\hline $\mathrm{C}(53)-\mathrm{C}(54)$ & $1.384(8)$ & $\mathrm{P}(4)-\mathrm{Pt}(2)-\mathrm{P}(3)$ & $171.39(4)$ \\
\hline$C(55)-C(56)$ & $1.367(7)$ & $\mathrm{P}(5)-\mathrm{Pt}(2)-\mathrm{P}(3)$ & $102.32(4)$ \\
\hline$C(55)-C(60)$ & $1.391(7)$ & $\mathrm{C}(4)-\mathrm{P}(1)-\mathrm{C}(10)$ & $106.9(2)$ \\
\hline$C(56)-C(57)$ & $1.399(7)$ & $\mathrm{C}(4)-\mathrm{P}(1)-\mathrm{C}(2)$ & $105.6(2)$ \\
\hline $\mathrm{C}(57)-\mathrm{C}(58)$ & $1.348(9)$ & $\mathrm{C}(10)-\mathrm{P}(1)-\mathrm{C}(2)$ & $105.7(2)$ \\
\hline $\mathrm{C}(58)-\mathrm{C}(59)$ & $1.342(11)$ & $\mathrm{C}(4)-\mathrm{P}(1)-\mathrm{Pt}(1)$ & $114.53(15)$ \\
\hline $\mathrm{C}(59)-\mathrm{C}(60)$ & $1.398(9)$ & $\mathrm{C}(10)-\mathrm{P}(1)-\mathrm{Pt}(1)$ & $114.34(16)$ \\
\hline $\mathrm{C}(61)-\mathrm{C}(62)$ & $1.367(7)$ & $\mathrm{C}(2)-\mathrm{P}(1)-\mathrm{Pt}(1)$ & $109.06(15)$ \\
\hline$C(61)-C(66)$ & $1.398(7)$ & $\mathrm{C}(22)-\mathrm{P}(2)-\mathrm{C}(16)$ & $107.0(2)$ \\
\hline$C(62)-C(63)$ & $1.369(8)$ & $\mathrm{C}(22)-\mathrm{P}(2)-\mathrm{C}(3)$ & $101.6(2)$ \\
\hline$C(63)-C(64)$ & $1.366(9)$ & $\mathrm{C}(16)-\mathrm{P}(2)-\mathrm{C}(3)$ & $101.2(2)$ \\
\hline$C(64)-C(65)$ & $1.349(9)$ & $\mathrm{C}(22)-\mathrm{P}(2)-\mathrm{Pt}(1)$ & $114.39(16)$ \\
\hline$C(65)-C(66)$ & $1.408(7)$ & $\mathrm{C}(16)-\mathrm{P}(2)-\mathrm{Pt}(1)$ & $123.55(15)$ \\
\hline$C(67)-C(68)$ & $1.398(15)$ & $\mathrm{C}(3)-\mathrm{P}(2)-\operatorname{Pt}(1)$ & $106.01(15)$ \\
\hline$C(68)-C(69)$ & 1.3900 & $\mathrm{C}(28)-\mathrm{P}(3)-\mathrm{C}(34)$ & $98.9(2)$ \\
\hline $\mathrm{C}(68)-\mathrm{C}(73)$ & 1.3900 & $\mathrm{C}(28)-\mathrm{P}(3)-\mathrm{Pt}(1)$ & $109.70(15)$ \\
\hline $\mathrm{C}(69)-\mathrm{C}(70)$ & 1.3900 & $\mathrm{C}(34)-\mathrm{P}(3)-\mathrm{Pt}(1)$ & $114.72(14)$ \\
\hline$C(70)-C(71)$ & 1.3900 & $\mathrm{C}(28)-\mathrm{P}(3)-\mathrm{Pt}(2)$ & $115.95(14)$ \\
\hline
\end{tabular}




\begin{tabular}{|c|c|c|c|}
\hline $\mathrm{C}(34)-\mathrm{P}(3)-\mathrm{Pt}(2)$ & $110.03(16)$ & $\mathrm{C}(14)-\mathrm{C}(15)-\mathrm{C}(10)$ & $121.8(5)$ \\
\hline $\operatorname{Pt}(1)-\mathrm{P}(3)-\mathrm{Pt}(2)$ & $107.61(5)$ & $\mathrm{C}(21)-\mathrm{C}(16)-\mathrm{C}(17)$ & $118.9(4)$ \\
\hline C(49)-P(4)-C(43) & $104.8(2)$ & $\mathrm{C}(21)-\mathrm{C}(16)-\mathrm{P}(2)$ & $118.2(4)$ \\
\hline C(49)-P(4)-C(41) & $103.6(2)$ & $\mathrm{C}(17)-\mathrm{C}(16)-\mathrm{P}(2)$ & $122.9(4)$ \\
\hline C(43)-P(4)-C(41) & $108.0(2)$ & $\mathrm{C}(18)-\mathrm{C}(17)-\mathrm{C}(16)$ & $119.9(5)$ \\
\hline C(49)-P(4)-Pt(2) & $113.00(16)$ & $\mathrm{C}(19)-\mathrm{C}(18)-\mathrm{C}(17)$ & $120.7(5)$ \\
\hline C(43)-P(4)-Pt(2) & $118.27(15)$ & $\mathrm{C}(18)-\mathrm{C}(19)-\mathrm{C}(20)$ & $120.3(5)$ \\
\hline $\mathrm{C}(41)-\mathrm{P}(4)-\mathrm{Pt}(2)$ & $108.05(15)$ & $C(19)-C(20)-C(21)$ & $119.5(5)$ \\
\hline C(55)-P(5)-C(61) & $109.2(2)$ & $C(16)-C(21)-C(20)$ & $120.6(5)$ \\
\hline $\mathrm{C}(55)-\mathrm{P}(5)-\mathrm{C}(42)$ & $101.4(2)$ & $\mathrm{C}(27)-\mathrm{C}(22)-\mathrm{C}(23)$ & $118.5(5)$ \\
\hline $\mathrm{C}(61)-\mathrm{P}(5)-\mathrm{C}(42)$ & $101.1(2)$ & $\mathrm{C}(27)-\mathrm{C}(22)-\mathrm{P}(2)$ & 119.1(4) \\
\hline C(55)-P(5)-Pt(2) & $114.65(17)$ & $\mathrm{C}(23)-\mathrm{C}(22)-\mathrm{P}(2)$ & $122.2(4)$ \\
\hline C(61)-P(5)-Pt(2) & $120.67(16)$ & $\mathrm{C}(24)-\mathrm{C}(23)-\mathrm{C}(22)$ & $120.8(5)$ \\
\hline $\mathrm{C}(42)-\mathrm{P}(5)-\mathrm{Pt}(2)$ & $106.98(15)$ & $\mathrm{C}(25)-\mathrm{C}(24)-\mathrm{C}(23)$ & $120.5(5)$ \\
\hline $\mathrm{C}(3)-\mathrm{C}(2)-\mathrm{P}(1)$ & $107.8(3)$ & $\mathrm{C}(24)-\mathrm{C}(25)-\mathrm{C}(26)$ & $120.5(5)$ \\
\hline $\mathrm{C}(2)-\mathrm{C}(3)-\mathrm{P}(2)$ & $109.5(3)$ & $\mathrm{C}(25)-\mathrm{C}(26)-\mathrm{C}(27)$ & $119.6(5)$ \\
\hline C(9)-C(4)-C(5) & $119.4(4)$ & $\mathrm{C}(22)-\mathrm{C}(27)-\mathrm{C}(26)$ & $120.1(5)$ \\
\hline C(9)-C(4)-P(1) & $117.1(4)$ & $\mathrm{C}(33)-\mathrm{C}(28)-\mathrm{C}(29)$ & $117.8(4)$ \\
\hline C(5)-C(4)-P(1) & $123.4(4)$ & $\mathrm{C}(33)-\mathrm{C}(28)-\mathrm{P}(3)$ & $123.1(3)$ \\
\hline$C(6)-C(5)-C(4)$ & $118.3(5)$ & $\mathrm{C}(29)-\mathrm{C}(28)-\mathrm{P}(3)$ & $119.0(4)$ \\
\hline$C(7)-C(6)-C(5)$ & $120.8(6)$ & $\mathrm{C}(30)-\mathrm{C}(29)-\mathrm{C}(28)$ & $120.4(5)$ \\
\hline $\mathrm{C}(6)-\mathrm{C}(7)-\mathrm{C}(8)$ & $121.9(5)$ & $\mathrm{C}(31)-\mathrm{C}(30)-\mathrm{C}(29)$ & $120.9(5)$ \\
\hline C(9)-C(8)-C(7) & $118.3(6)$ & $\mathrm{C}(30)-\mathrm{C}(31)-\mathrm{C}(32)$ & $119.2(5)$ \\
\hline C(8)-C(9)-C(4) & $121.2(5)$ & $\mathrm{C}(33)-\mathrm{C}(32)-\mathrm{C}(31)$ & $119.9(5)$ \\
\hline $\mathrm{C}(11)-\mathrm{C}(10)-\mathrm{C}(15)$ & $118.5(5)$ & $\mathrm{C}(28)-\mathrm{C}(33)-\mathrm{C}(32)$ & $121.6(5)$ \\
\hline $\mathrm{C}(11)-\mathrm{C}(10)-\mathrm{P}(1)$ & $121.8(4)$ & C(35)-C(34)-C(39) & $118.8(5)$ \\
\hline $\mathrm{C}(15)-\mathrm{C}(10)-\mathrm{P}(1)$ & $119.6(4)$ & $\mathrm{C}(35)-\mathrm{C}(34)-\mathrm{P}(3)$ & $122.2(4)$ \\
\hline $\mathrm{C}(10)-\mathrm{C}(11)-\mathrm{C}(12)$ & $119.6(5)$ & $\mathrm{C}(39)-\mathrm{C}(34)-\mathrm{P}(3)$ & $119.0(4)$ \\
\hline $\mathrm{C}(13)-\mathrm{C}(12)-\mathrm{C}(11)$ & $119.5(5)$ & $\mathrm{C}(34)-\mathrm{C}(35)-\mathrm{C}(36)$ & $121.0(6)$ \\
\hline $\mathrm{C}(14)-\mathrm{C}(13)-\mathrm{C}(12)$ & $121.0(5)$ & $\mathrm{C}(37)-\mathrm{C}(36)-\mathrm{C}(35)$ & $120.1(6)$ \\
\hline$C(13)-C(14)-C(15)$ & $119.4(6)$ & $\mathrm{C}(36)-\mathrm{C}(37)-\mathrm{C}(38)$ & $120.5(5)$ \\
\hline
\end{tabular}




\begin{tabular}{|c|c|c|c|}
\hline $\mathrm{C}(37)-\mathrm{C}(38)-\mathrm{C}(39)$ & $119.0(6)$ & $\mathrm{C}(60)-\mathrm{C}(55)-\mathrm{P}(5)$ & $120.9(4)$ \\
\hline $\mathrm{C}(34)-\mathrm{C}(39)-\mathrm{C}(38)$ & $120.7(5)$ & $\mathrm{C}(55)-\mathrm{C}(56)-\mathrm{C}(57)$ & $119.8(5)$ \\
\hline $\mathrm{C}(42)-\mathrm{C}(41)-\mathrm{P}(4)$ & $105.3(3)$ & $\mathrm{C}(58)-\mathrm{C}(57)-\mathrm{C}(56)$ & $120.7(6)$ \\
\hline $\mathrm{C}(41)-\mathrm{C}(42)-\mathrm{P}(5)$ & $108.9(3)$ & $\mathrm{C}(59)-\mathrm{C}(58)-\mathrm{C}(57)$ & $119.6(6)$ \\
\hline $\mathrm{C}(44)-\mathrm{C}(43)-\mathrm{C}(48)$ & $119.6(4)$ & $\mathrm{C}(58)-\mathrm{C}(59)-\mathrm{C}(60)$ & $122.0(6)$ \\
\hline $\mathrm{C}(44)-\mathrm{C}(43)-\mathrm{P}(4)$ & $120.2(3)$ & $\mathrm{C}(55)-\mathrm{C}(60)-\mathrm{C}(59)$ & $118.2(6)$ \\
\hline $\mathrm{C}(48)-\mathrm{C}(43)-\mathrm{P}(4)$ & $120.0(4)$ & $\mathrm{C}(62)-\mathrm{C}(61)-\mathrm{C}(66)$ & $118.4(5)$ \\
\hline $\mathrm{C}(43)-\mathrm{C}(44)-\mathrm{C}(45)$ & $120.0(5)$ & $\mathrm{C}(62)-\mathrm{C}(61)-\mathrm{P}(5)$ & $126.3(4)$ \\
\hline $\mathrm{C}(44)-\mathrm{C}(45)-\mathrm{C}(46)$ & $119.7(5)$ & $\mathrm{C}(66)-\mathrm{C}(61)-\mathrm{P}(5)$ & $115.3(4)$ \\
\hline $\mathrm{C}(47)-\mathrm{C}(46)-\mathrm{C}(45)$ & $120.3(5)$ & $\mathrm{C}(61)-\mathrm{C}(62)-\mathrm{C}(63)$ & $120.8(6)$ \\
\hline $\mathrm{C}(46)-\mathrm{C}(47)-\mathrm{C}(48)$ & $120.0(5)$ & $\mathrm{C}(64)-\mathrm{C}(63)-\mathrm{C}(62)$ & $121.2(6)$ \\
\hline $\mathrm{C}(43)-\mathrm{C}(48)-\mathrm{C}(47)$ & $120.4(5)$ & $\mathrm{C}(65)-\mathrm{C}(64)-\mathrm{C}(63)$ & $119.9(6)$ \\
\hline $\mathrm{C}(54)-\mathrm{C}(49)-\mathrm{C}(50)$ & $118.7(4)$ & $\mathrm{C}(64)-\mathrm{C}(65)-\mathrm{C}(66)$ & $120.0(6)$ \\
\hline $\mathrm{C}(54)-\mathrm{C}(49)-\mathrm{P}(4)$ & $121.2(4)$ & $\mathrm{C}(61)-\mathrm{C}(66)-\mathrm{C}(65)$ & $119.7(5)$ \\
\hline $\mathrm{C}(50)-\mathrm{C}(49)-\mathrm{P}(4)$ & $120.1(4)$ & $\mathrm{C}(69)-\mathrm{C}(68)-\mathrm{C}(73)$ & 120.0 \\
\hline $\mathrm{C}(51)-\mathrm{C}(50)-\mathrm{C}(49)$ & $120.8(5)$ & $\mathrm{C}(69)-\mathrm{C}(68)-\mathrm{C}(67)$ & $98.8(10)$ \\
\hline $\mathrm{C}(52)-\mathrm{C}(51)-\mathrm{C}(50)$ & $119.0(5)$ & $\mathrm{C}(73)-\mathrm{C}(68)-\mathrm{C}(67)$ & $139.5(10)$ \\
\hline $\mathrm{C}(53)-\mathrm{C}(52)-\mathrm{C}(51)$ & $121.0(5)$ & $\mathrm{C}(70)-\mathrm{C}(69)-\mathrm{C}(68)$ & 120.0 \\
\hline $\mathrm{C}(52)-\mathrm{C}(53)-\mathrm{C}(54)$ & $120.2(6)$ & $\mathrm{C}(69)-\mathrm{C}(70)-\mathrm{C}(71)$ & 120.0 \\
\hline$C(53)-C(54)-C(49)$ & $120.2(5)$ & $\mathrm{C}(70)-\mathrm{C}(71)-\mathrm{C}(72)$ & 120.0 \\
\hline$C(56)-C(55)-C(60)$ & $119.6(5)$ & $\mathrm{C}(73)-\mathrm{C}(72)-\mathrm{C}(71)$ & 120.0 \\
\hline $\mathrm{C}(56)-\mathrm{C}(55)-\mathrm{P}(5)$ & 119.2(4) & $\mathrm{C}(72)-\mathrm{C}(73)-\mathrm{C}(68)$ & 120.0 \\
\hline
\end{tabular}

Symmetry transformations used to generate equivalent atoms: 
Table 4. Anisotropic displacement parameters $\left(\AA^{2} \mathrm{x} 10^{3}\right)$ for $\left[\left(\mathrm{Pt}(\mathrm{dppe})(\mathrm{Me})_{2}\left(\mu-\mathrm{PPh}_{2}\right)\right][\mathrm{OH}] \cdot 2.5 \mathrm{C}_{7} \mathrm{H}_{8}\right.$, glu241. The anisotropic displacement factor exponent takes the form: $-2 \pi^{2}\left[h^{2} a^{* 2} U^{11}+\ldots+2 h k a^{*} b^{*} U^{12}\right]$

\begin{tabular}{|c|c|c|c|c|c|c|}
\hline & $\mathrm{U}^{11}$ & $\mathrm{U}^{22}$ & $\mathrm{U}^{33}$ & $\mathrm{U}^{23}$ & $\mathrm{U}^{13}$ & $\mathrm{U}^{12}$ \\
\hline $\operatorname{Pt}(1)$ & $30(1)$ & $24(1)$ & $25(1)$ & $5(1)$ & $17(1)$ & $10(1)$ \\
\hline $\operatorname{Pt}(2)$ & $29(1)$ & $24(1)$ & $23(1)$ & $5(1)$ & $16(1)$ & $10(1)$ \\
\hline $\mathrm{P}(1)$ & $35(1)$ & $26(1)$ & $27(1)$ & $6(1)$ & $19(1)$ & $14(1)$ \\
\hline $\mathrm{P}(2)$ & $33(1)$ & $26(1)$ & $24(1)$ & $4(1)$ & $16(1)$ & $9(1)$ \\
\hline $\mathrm{P}(3)$ & $32(1)$ & $24(1)$ & $28(1)$ & $5(1)$ & $19(1)$ & $10(1)$ \\
\hline $\mathrm{P}(4)$ & $30(1)$ & $28(1)$ & $25(1)$ & $5(1)$ & $17(1)$ & $9(1)$ \\
\hline $\mathrm{P}(5)$ & $36(1)$ & $24(1)$ & $25(1)$ & $4(1)$ & $19(1)$ & $9(1)$ \\
\hline $\mathrm{C}(1)$ & $33(2)$ & $33(2)$ & $33(2)$ & $11(2)$ & $19(2)$ & $11(2)$ \\
\hline$C(2)$ & $42(3)$ & $32(2)$ & $31(2)$ & $6(2)$ & $24(2)$ & $13(2)$ \\
\hline$C(3)$ & $47(3)$ & $26(2)$ & $25(2)$ & $2(2)$ & $22(2)$ & $9(2)$ \\
\hline$C(4)$ & $28(2)$ & $42(3)$ & $34(2)$ & $16(2)$ & $20(2)$ & $17(2)$ \\
\hline$C(5)$ & $39(3)$ & $42(3)$ & $50(3)$ & $23(2)$ & $29(2)$ & $18(2)$ \\
\hline$C(6)$ & $41(3)$ & $76(4)$ & $76(4)$ & $56(4)$ & $33(3)$ & $23(3)$ \\
\hline$C(7)$ & $30(3)$ & $88(5)$ & $64(4)$ & $51(4)$ & $13(3)$ & $4(3)$ \\
\hline$C(8)$ & $29(3)$ & $73(4)$ & $42(3)$ & $24(3)$ & $15(2)$ & $3(3)$ \\
\hline$C(9)$ & $35(3)$ & $46(3)$ & $38(3)$ & $15(2)$ & $24(2)$ & $14(2)$ \\
\hline$C(10)$ & $52(3)$ & $27(2)$ & $23(2)$ & $10(2)$ & $16(2)$ & $13(2)$ \\
\hline $\mathrm{C}(11)$ & $69(4)$ & $30(3)$ & $41(3)$ & $10(2)$ & $36(3)$ & $13(2)$ \\
\hline$C(12)$ & $106(5)$ & $30(3)$ & $28(3)$ & $3(2)$ & $28(3)$ & $7(3)$ \\
\hline$C(13)$ & $58(4)$ & $41(3)$ & $37(3)$ & $12(2)$ & $8(3)$ & $-5(3)$ \\
\hline$C(14)$ & $50(3)$ & $44(3)$ & $42(3)$ & $16(3)$ & $6(3)$ & $4(3)$ \\
\hline$C(15)$ & $38(3)$ & $33(3)$ & $43(3)$ & $8(2)$ & $13(2)$ & $7(2)$ \\
\hline$C(16)$ & $36(2)$ & $35(2)$ & $29(2)$ & $12(2)$ & $24(2)$ & $13(2)$ \\
\hline $\mathrm{C}(17)$ & $42(3)$ & $36(3)$ & $39(3)$ & $13(2)$ & $20(2)$ & $14(2)$ \\
\hline $\mathrm{C}(18)$ & $55(3)$ & $44(3)$ & $52(3)$ & $23(3)$ & $25(3)$ & $19(3)$ \\
\hline C(19) & $44(3)$ & $64(4)$ & $46(3)$ & $31(3)$ & $17(3)$ & $22(3)$ \\
\hline$C(20)$ & $42(3)$ & $58(4)$ & $39(3)$ & $19(3)$ & $21(2)$ & $7(3)$ \\
\hline $\mathrm{C}(21)$ & $38(3)$ & $40(3)$ & $34(3)$ & $14(2)$ & $21(2)$ & $8(2)$ \\
\hline $\mathrm{C}(22)$ & $34(2)$ & $27(2)$ & $34(2)$ & $4(2)$ & $20(2)$ & $8(2)$ \\
\hline
\end{tabular}




\begin{tabular}{|c|c|c|c|c|c|c|}
\hline$C(23)$ & $41(3)$ & $38(3)$ & $38(3)$ & $6(2)$ & $22(2)$ & $9(2)$ \\
\hline$C(24)$ & $49(3)$ & $46(3)$ & $58(4)$ & $14(3)$ & $34(3)$ & $7(3)$ \\
\hline$C(25)$ & $40(3)$ & $40(3)$ & $72(4)$ & $4(3)$ & $26(3)$ & $4(2)$ \\
\hline$C(26)$ & $46(3)$ & $35(3)$ & $53(3)$ & $-2(3)$ & $13(3)$ & $11(2)$ \\
\hline$C(27)$ & $39(3)$ & $37(3)$ & $32(3)$ & $3(2)$ & $13(2)$ & $12(2)$ \\
\hline$C(28)$ & $36(3)$ & $25(2)$ & $32(2)$ & 2(2) & $24(2)$ & $3(2)$ \\
\hline$C(29)$ & $46(3)$ & $31(3)$ & $36(3)$ & $4(2)$ & $20(2)$ & $12(2)$ \\
\hline$C(30)$ & $55(3)$ & $32(3)$ & $42(3)$ & $-3(2)$ & $24(3)$ & $8(2)$ \\
\hline$C(31)$ & $56(3)$ & $42(3)$ & $32(3)$ & $-4(2)$ & $18(3)$ & $-3(3)$ \\
\hline $\mathrm{C}(32)$ & $37(3)$ & 49(3) & $36(3)$ & $7(2)$ & $10(2)$ & $6(2)$ \\
\hline$C(33)$ & $39(3)$ & $37(3)$ & $34(3)$ & $0(2)$ & $17(2)$ & $8(2)$ \\
\hline$C(34)$ & $48(3)$ & $26(2)$ & $36(3)$ & $9(2)$ & $30(2)$ & $14(2)$ \\
\hline$C(35)$ & $58(3)$ & 41(3) & $45(3)$ & $20(2)$ & $35(3)$ & $22(2)$ \\
\hline$C(36)$ & $75(4)$ & $53(4)$ & $54(4)$ & 27(3) & $42(3)$ & $31(3)$ \\
\hline$C(37)$ & $111(6)$ & $37(3)$ & $57(4)$ & 26(3) & $58(4)$ & $35(3)$ \\
\hline$C(38)$ & $88(5)$ & $31(3)$ & $53(3)$ & $8(2)$ & $50(3)$ & $7(3)$ \\
\hline C(39) & $61(3)$ & $26(2)$ & 41(3) & $6(2)$ & $34(3)$ & $13(2)$ \\
\hline $\mathrm{C}(40)$ & $34(3)$ & $47(3)$ & $27(2)$ & $4(2)$ & $21(2)$ & $10(2)$ \\
\hline $\mathrm{C}(41)$ & $32(2)$ & $28(2)$ & $31(2)$ & $5(2)$ & $24(2)$ & $13(2)$ \\
\hline$C(42)$ & $35(2)$ & $30(2)$ & $31(2)$ & $8(2)$ & $21(2)$ & $8(2)$ \\
\hline$C(43)$ & $26(2)$ & $34(2)$ & $25(2)$ & $6(2)$ & $14(2)$ & $6(2)$ \\
\hline$C(44)$ & $43(3)$ & $39(3)$ & $32(3)$ & $5(2)$ & $19(2)$ & $18(2)$ \\
\hline$C(45)$ & $40(3)$ & $52(3)$ & $34(3)$ & $13(2)$ & $18(2)$ & $19(2)$ \\
\hline $\mathrm{C}(46)$ & $34(3)$ & $54(3)$ & $30(3)$ & $6(2)$ & $15(2)$ & $3(2)$ \\
\hline$C(47)$ & $46(3)$ & $40(3)$ & $35(3)$ & $5(2)$ & $18(2)$ & $3(2)$ \\
\hline $\mathrm{C}(48)$ & $36(3)$ & $34(3)$ & $37(3)$ & $7(2)$ & $11(2)$ & $8(2)$ \\
\hline $\mathrm{C}(49)$ & $40(3)$ & $26(2)$ & $28(2)$ & $1(2)$ & $19(2)$ & $10(2)$ \\
\hline$C(50)$ & $38(3)$ & $34(3)$ & $45(3)$ & $14(2)$ & $23(2)$ & $14(2)$ \\
\hline$C(51)$ & $54(4)$ & 41(3) & $69(4)$ & 21(3) & $30(3)$ & $22(3)$ \\
\hline$C(52)$ & $71(4)$ & $40(3)$ & $77(4)$ & 28(3) & $45(4)$ & $23(3)$ \\
\hline$C(53)$ & $66(4)$ & $37(3)$ & 73(4) & 17(3) & $48(3)$ & $13(3)$ \\
\hline$C(54)$ & $44(3)$ & $36(3)$ & $52(3)$ & $12(2)$ & $30(3)$ & $12(2)$ \\
\hline $\mathrm{C}(55)$ & $45(3)$ & $29(2)$ & $50(3)$ & 1(2) & $35(3)$ & $9(2)$ \\
\hline
\end{tabular}




\begin{tabular}{lllllll}
$\mathrm{C}(56)$ & $33(3)$ & $34(3)$ & $55(3)$ & $15(2)$ & $20(2)$ & $10(2)$ \\
$\mathrm{C}(57)$ & $46(3)$ & $35(3)$ & $78(4)$ & $14(3)$ & $26(3)$ & $14(2)$ \\
$\mathrm{C}(58)$ & $82(5)$ & $35(3)$ & $120(7)$ & $16(4)$ & $59(5)$ & $25(3)$ \\
$\mathrm{C}(59)$ & $106(6)$ & $37(3)$ & $120(7)$ & $0(4)$ & $87(5)$ & $25(4)$ \\
$\mathrm{C}(60)$ & $86(5)$ & $34(3)$ & $71(4)$ & $0(3)$ & $59(4)$ & $10(3)$ \\
$\mathrm{C}(61)$ & $35(3)$ & $44(3)$ & $26(2)$ & $9(2)$ & $16(2)$ & $2(2)$ \\
$\mathrm{C}(62)$ & $75(4)$ & $39(3)$ & $39(3)$ & $8(2)$ & $20(3)$ & $-3(3)$ \\
$\mathrm{C}(63)$ & $63(4)$ & $67(4)$ & $34(3)$ & $8(3)$ & $4(3)$ & $-8(3)$ \\
$\mathrm{C}(64)$ & $52(4)$ & $84(5)$ & $38(3)$ & $30(3)$ & $5(3)$ & $-4(3)$ \\
$\mathrm{C}(65)$ & $42(3)$ & $66(4)$ & $52(3)$ & $33(3)$ & $24(3)$ & $19(3)$ \\
$\mathrm{C}(66)$ & $36(3)$ & $45(3)$ & $39(3)$ & $10(2)$ & $21(2)$ & $13(2)$ \\
$\mathrm{O}(1)$ & $131(5)$ & $35(2)$ & $234(7)$ & $34(3)$ & $164(5)$ & $31(3)$ \\
& & & & & & \\
\hline
\end{tabular}


Table 5. Hydrogen coordinates $\left(\mathrm{x} 10^{4}\right)$ and isotropic displacement parameters $\left(\AA^{2} \times 10^{3}\right)$ for $\left[\left(\mathrm{Pt}(\mathrm{dppe})(\mathrm{Me})_{2}\left(\mu-\mathrm{PPh}_{2}\right)\right][\mathrm{OH}] \cdot 2.5 \mathrm{C}_{7} \mathrm{H}_{8}\right.$, glu241.

\begin{tabular}{|c|c|c|c|c|}
\hline & $\mathrm{x}$ & $\mathrm{y}$ & $\mathrm{z}$ & $\mathrm{U}(\mathrm{eq})$ \\
\hline $\mathrm{H}(1 \mathrm{~A})$ & 310 & 5334 & 3608 & 46 \\
\hline $\mathrm{H}(1 \mathrm{~B})$ & -788 & 5745 & 3560 & 46 \\
\hline $\mathrm{H}(1 \mathrm{C})$ & 382 & 6249 & 4203 & 46 \\
\hline $\mathrm{H}(2 \mathrm{~A})$ & 2300 & 5776 & 1900 & 39 \\
\hline $\mathrm{H}(2 \mathrm{~B})$ & 1672 & 4903 & 1226 & 39 \\
\hline $\mathrm{H}(3 \mathrm{~A})$ & 130 & 5520 & 639 & 37 \\
\hline $\mathrm{H}(3 \mathrm{~B})$ & 1183 & 6083 & 691 & 37 \\
\hline $\mathrm{H}(5 \mathrm{~A})$ & 1700 & 3712 & 2704 & 46 \\
\hline $\mathrm{H}(6 \mathrm{~A})$ & 3098 & 3517 & 3862 & 65 \\
\hline $\mathrm{H}(7 \mathrm{~A})$ & 4183 & 4630 & 4930 & 67 \\
\hline $\mathrm{H}(8 \mathrm{~A})$ & 3975 & 5990 & 4890 & 55 \\
\hline $\mathrm{H}(9 \mathrm{~A})$ & 2617 & 6211 & 3741 & 43 \\
\hline $\mathrm{H}(11 \mathrm{~A})$ & 960 & 3601 & 1218 & 51 \\
\hline $\mathrm{H}(12 \mathrm{~A})$ & -308 & 2362 & 565 & 66 \\
\hline $\mathrm{H}(13 \mathrm{~A})$ & -2064 & 2351 & 635 & 58 \\
\hline $\mathrm{H}(14 \mathrm{~A})$ & -2557 & 3527 & 1366 & 57 \\
\hline $\mathrm{H}(15 \mathrm{~A})$ & -1318 & 4742 & 2011 & 47 \\
\hline $\mathrm{H}(17 \mathrm{~A})$ & 72 & 8369 & 1238 & 44 \\
\hline $\mathrm{H}(18 \mathrm{~A})$ & -1309 & 8729 & 207 & 56 \\
\hline $\mathrm{H}(19 \mathrm{~A})$ & -2800 & 7754 & -732 & 57 \\
\hline $\mathrm{H}(20 \mathrm{~A})$ & -2953 & 6409 & -627 & 53 \\
\hline $\mathrm{H}(21 \mathrm{~A})$ & -1558 & 6028 & 397 & 42 \\
\hline $\mathrm{H}(23 \mathrm{~A})$ & 1585 & 7728 & 943 & 45 \\
\hline $\mathrm{H}(24 \mathrm{~A})$ & 3230 & 8606 & 1434 & 57 \\
\hline $\mathrm{H}(25 \mathrm{~A})$ & 4341 & 8984 & 2835 & 62 \\
\hline $\mathrm{H}(26 \mathrm{~A})$ & 3866 & 8427 & 3759 & 59 \\
\hline $\mathrm{H}(27 \mathrm{~A})$ & 2177 & 7549 & 3280 & 45 \\
\hline
\end{tabular}




\begin{tabular}{|c|c|c|c|c|}
\hline $\mathrm{H}(29 \mathrm{~A})$ & -772 & 8958 & 4462 & 45 \\
\hline $\mathrm{H}(30 \mathrm{~A})$ & 331 & 9715 & 5826 & 53 \\
\hline $\mathrm{H}(31 \mathrm{~A})$ & 1910 & 9247 & 6547 & 55 \\
\hline $\mathrm{H}(32 \mathrm{~A})$ & 2437 & 8020 & 5877 & 51 \\
\hline $\mathrm{H}(33 \mathrm{~A})$ & 1340 & 7260 & 4521 & 45 \\
\hline $\mathrm{H}(35 \mathrm{~A})$ & -2493 & 8060 & 2156 & 50 \\
\hline $\mathrm{H}(36 \mathrm{~A})$ & -2631 & 9196 & 1634 & 64 \\
\hline $\mathrm{H}(37 \mathrm{~A})$ & -1070 & 10104 & 1941 & 69 \\
\hline $\mathrm{H}(38 \mathrm{~A})$ & 642 & 9911 & 2810 & 62 \\
\hline $\mathrm{H}(39 \mathrm{~A})$ & 772 & 8767 & 3342 & 47 \\
\hline $\mathrm{H}(40 \mathrm{~A})$ & -3423 & 5953 & 1544 & 52 \\
\hline $\mathrm{H}(40 \mathrm{~B})$ & -2125 & 5930 & 1811 & 52 \\
\hline $\mathrm{H}(40 \mathrm{C})$ & -2636 & 6723 & 1580 & 52 \\
\hline $\mathrm{H}(41 \mathrm{~A})$ & -4970 & 7137 & 3455 & 33 \\
\hline $\mathrm{H}(41 \mathrm{~B})$ & -5341 & 6261 & 3556 & 33 \\
\hline $\mathrm{H}(42 \mathrm{~A})$ & -3580 & 6309 & 4568 & 35 \\
\hline $\mathrm{H}(42 \mathrm{~B})$ & -4029 & 7166 & 4866 & 35 \\
\hline $\mathrm{H}(44 \mathrm{~A})$ & -5692 & 6981 & 1949 & 45 \\
\hline $\mathrm{H}(45 \mathrm{~A})$ & -6957 & 6708 & 577 & 49 \\
\hline $\mathrm{H}(46 \mathrm{~A})$ & -7131 & 5420 & -408 & 48 \\
\hline $\mathrm{H}(47 \mathrm{~A})$ & -6082 & 4415 & -22 & 49 \\
\hline $\mathrm{H}(48 \mathrm{~A})$ & -4859 & 4672 & 1357 & 44 \\
\hline $\mathrm{H}(50 \mathrm{~A})$ & -2314 & 5136 & 3219 & 44 \\
\hline $\mathrm{H}(51 \mathrm{~A})$ & -2072 & 3876 & 3543 & 61 \\
\hline $\mathrm{H}(52 \mathrm{~A})$ & -3573 & 3077 & 3535 & 66 \\
\hline $\mathrm{H}(53 \mathrm{~A})$ & -5305 & 3498 & 3174 & 63 \\
\hline $\mathrm{H}(54 \mathrm{~A})$ & -5557 & 4752 & 2859 & 49 \\
\hline $\mathrm{H}(56 \mathrm{~A})$ & -2882 & 8608 & 3501 & 47 \\
\hline $\mathrm{H}(57 \mathrm{~A})$ & -3461 & 9925 & 3670 & 63 \\
\hline $\mathrm{H}(58 \mathrm{~A})$ & -3829 & 10566 & 4840 & 88 \\
\hline $\mathrm{H}(59 \mathrm{~A})$ & -3772 & 9874 & 5791 & 95 \\
\hline $\mathrm{H}(60 \mathrm{~A})$ & -3161 & 8569 & 5680 & 70 \\
\hline $\mathrm{H}(62 \mathrm{~A})$ & -1155 & 8528 & 6086 & 63 \\
\hline
\end{tabular}




\begin{tabular}{lrrrr}
$\mathrm{H}(63 \mathrm{~A})$ & 192 & 8304 & 7217 & 72 \\
$\mathrm{H}(64 \mathrm{~A})$ & 649 & 6988 & 7195 & 71 \\
$\mathrm{H}(65 \mathrm{~A})$ & -152 & 5895 & 5999 & 58 \\
$\mathrm{H}(66 \mathrm{~A})$ & -1519 & 6102 & 4822 & 46 \\
$\mathrm{H}(67 \mathrm{~A})$ & -3028 & 610 & 1120 & 485 \\
$\mathrm{H}(67 \mathrm{~B})$ & -3236 & 1098 & 1944 & 485 \\
$\mathrm{H}(67 \mathrm{C})$ & -2335 & 1501 & 1680 & 485 \\
$\mathrm{H}(69 \mathrm{~A})$ & -3731 & 512 & 145 & 196 \\
$\mathrm{H}(70 \mathrm{~A})$ & -4739 & 895 & -1039 & 240 \\
$\mathrm{H}(71 \mathrm{~A})$ & -5465 & 2153 & -889 & 167 \\
$\mathrm{H}(72 \mathrm{~A})$ & -5182 & 3029 & 446 & 216 \\
$\mathrm{H}(73 \mathrm{~A})$ & -4174 & 2646 & 1631 & 144 \\
& & & & \\
\hline
\end{tabular}

\section{Experimental for $\left[\left(\mathrm{Pt}(\mathrm{dppe})(\mathrm{Me})_{2}\left(\mu-\mathrm{PPh}_{2}\right)\right][\mathrm{OH}] \cdot 2.5 \mathrm{C}_{7} \mathrm{H}_{8}\right.$, glu241.}

A brown crystal was mounted on a cryoloop with Paratone-N oil and cooled to $100 \mathrm{~K}$ under a stream of nitrogen gas. Data was collected at $100 \mathrm{~K}$ with a Bruker SMART Apex system using Mo K alpha radiation. Data was corrected for adsorption using SADABS and structure solved by direct methods. All non-hydrogen atoms of the main molecule were refined as anisotropic by full matrix least squares on F2. All hydrogen atoms were placed in calculated positions. The ring system of a toluene molecule was fixed and refined isotropically. The counter ion was found to be a hydroxide ion and the oxygen atom was refined anisotropically. The $\mathrm{H}$ atom of $\mathrm{OH}$ could not be located with any degree of certainty. Platon program Squeeze was used to treat the remaining unresolved solvent. The program found 151 e/unit cell which was associated with three $\mathrm{C}_{7} \mathrm{H}_{8}$ units and $2 \mathrm{H}$ atoms of the hydroxide ions.

\section{References}

1. Several phosphines $\mathrm{R}_{2} \mathrm{PCH}_{2} \mathrm{CH}_{2} \mathrm{X}(3)$ were prepared, differing in $\mathrm{X}$ and $\mathrm{PR}_{2}$. Numbering scheme: for $\mathrm{X}=\mathrm{CO}_{2} t-\mathrm{Bu}, \mathrm{PPh}_{2}=\mathbf{a}, \mathrm{PPh}(i-\mathrm{Bu})=\mathbf{b}, \mathrm{PMeIs}=\mathbf{c}$. Numbering for $\mathrm{X}=\mathrm{CN}$ is similar but with an additional '. For example, $\mathrm{Ph}_{2} \mathrm{PCH}_{2} \mathrm{CH}_{2} \mathrm{CO}_{2} t-\mathrm{Bu}$ is $\mathbf{3 a}, \mathrm{MeIsPCH}_{2} \mathrm{CH}_{2} \mathrm{CO}_{2} t-\mathrm{Bu}$ is $\mathbf{3 c}$, and $\mathrm{Ph}_{2} \mathrm{PCH}_{2} \mathrm{CH}_{2} \mathrm{CN}$ is 3a'. Similarly, several phosphines $\mathrm{R}_{2} \mathrm{P}\left(\mathrm{CH}_{2} \mathrm{CH}(\mathrm{X})\right)_{\mathrm{n}} \mathrm{CH}_{2} \mathrm{CH}_{2} \mathrm{X}$ (7) were prepared, differing in $\mathrm{n}, \mathrm{X}$, and $\mathrm{PR}_{2}$. Numbering scheme: for $\mathrm{X}=\mathrm{CO}_{2} t-\mathrm{Bu}, \mathrm{PPh}_{2}=\mathbf{a}, \mathrm{PPh}(i-\mathrm{Bu})=\mathbf{b}, \mathrm{PMeIs}=\mathbf{c} ; \mathrm{n}$

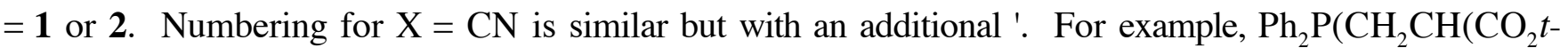


$\mathrm{Bu}) \mathrm{CH}_{2} \mathrm{CH}_{2} \mathrm{CO}_{2} t-\mathrm{Bu}$ is $\mathbf{7} \mathbf{a} \mathbf{1}, \quad \mathrm{MeIsP}\left(\mathrm{CH}_{2} \mathrm{CH}\left(\mathrm{CO}_{2} t-\mathrm{Bu}\right)\right)_{2} \mathrm{CH}_{2} \mathrm{CH}_{2} \mathrm{CO}_{2} t-\mathrm{Bu}$ is $7 \mathbf{c} 2$, and $\mathrm{Ph}_{2} \mathrm{P}\left(\mathrm{CH}_{2} \mathrm{CH}(\mathrm{CN})\right)_{2} \mathrm{CH}_{2} \mathrm{CH}_{2} \mathrm{CN}$ is 7a1'.

2. Scriban, C.; Kovacik, I.; Glueck, D. S. Organometallics 2005, 24, 4871-4874.

3. Scriban, C.; Wicht, D. K.; Glueck, D. S.; Zakharov, L. N.; Golen, J. A.; Rheingold, A. L. Organometallics 2006, 25, 3370-3378.

4. (a) Clark, H. C.; Kapoor, P. N.; McMahon, I. J. J. Organomet. Chem. 1984, 265, 107-115. (b) Chaloner, P. A.; Broadwood-Strong, G. T. L. J. Chem. Soc., Dalton Trans. 1996, 1039-1043.

5. Appleton, T. G.; Bennett, M. A.; Tomkins, I. B. J. Chem. Soc., Dalton Trans. 1976, 439-446.

6. Wicht, D. K.; Paisner, S. N.; Lew, B. M.; Glueck, D. S.; Yap, G. P. A.; Liable-Sands, L. M.; Rheingold, A. L.; Haar, C. M.; Nolan, S. P. Organometallics 1998, 17, 652-660.

7. Bergamini, P.; Costa, E.; Cramer, P.; Hogg, J.; Orpen, A. G.; Pringle, P. G. Organometallics 1994, 13, 1058-1060. 